University of Tennessee Health Science Center

UTHSC Digital Commons

$12-2011$

\title{
Characterization of the Key Mouse Cochlear Developmental Genes for Auditory Hair Cell Regeneration
}

Zhiyong Liu

University of Tennessee Health Science Center

Follow this and additional works at: https://dc.uthsc.edu/dissertations

Part of the Medical Cell Biology Commons

\section{Recommended Citation}

Liu, Zhiyong , "Characterization of the Key Mouse Cochlear Developmental Genes for Auditory Hair Cell Regeneration" (2011). Theses and Dissertations (ETD). Paper 363. http://dx.doi.org/10.21007/ etd.cghs.2011.0189.

This Dissertation is brought to you for free and open access by the College of Graduate Health Sciences at UTHSC Digital Commons. It has been accepted for inclusion in Theses and Dissertations (ETD) by an authorized administrator of UTHSC Digital Commons. For more information, please contact jwelch30@uthsc.edu. 


\title{
Characterization of the Key Mouse Cochlear Developmental Genes for Auditory Hair Cell Regeneration
}

\author{
Abstract \\ Mammalian inner ear cochlear auditory hair cells (HCs) and adjacent supporting cells (SCs) are believed \\ to derive from the same progenitors during development. However, unlike SCs of nonmammalian \\ vertebrates, mammalian cochlear SCs cannot be converted into functional hair cells (HCs) after damage, \\ thus leading to permanent deafness. To entitle mammals with the ability to restore hearing capacity after \\ $\mathrm{HC}$ damage, we first achieved proliferation of SCs by acute ablation of p27 or Sox2. Secondly, we \\ overactivated Notch1 signaling in the mouse inner ear at different developmental stages, and found that \\ the ability of Notch signaling in generating new HCs declines after birth. Last, we achieved \\ reprogramming of neonatal and juvenile, but not adult, SCs into HCs, through ectopically expressing \\ Atoh1 in SCs. Taken together, Atoh1 is able to reprogram SCs into HCs, and inactivation of p27 or Sox2 \\ can achieve proliferation of SCs. This work builds the foundation for future $\mathrm{HC}$ regeneration studies. \\ Combining these two manipulations may represent a promising approach to recapitulate the cellular \\ events occurring in the process of $\mathrm{HC}$ regeneration in non-mammalian vertebrates. \\ Document Type \\ Dissertation \\ Degree Name \\ Doctor of Philosophy (PhD) \\ Program \\ Biomedical Sciences \\ Research Advisor \\ Jian Zuo, Ph.D. \\ Keywords \\ Atoh1, Cochlea, Hair cells, Regeneration, Sox2, Supporting cells \\ Subject Categories \\ Medical Cell Biology | Medical Sciences | Medicine and Health Sciences \\ Comments \\ Two year embargo expired December 2013
}




\title{
CHARACTERIZATION OF THE KEY MOUSE COCHLEAR DEVELOPMENTAL GENES FOR AUDITORY HAIR CELL REGENERATION
}

\author{
A Dissertation \\ Presented for \\ The Graduate Studies Council \\ The University of Tennessee \\ Health Science Center
}

\author{
In Partial Fulfillment \\ Of the Requirements for the Degree \\ Doctor of Philosophy \\ From The University of Tennessee
}

By

Zhiyong Liu

December 2011 
Copyright (C) 2011 by Zhiyong Liu. All rights reserved. 


\section{DEDICATION}

This dissertation is dedicated to my wife, Fang Lei, and my parents, Chenliang Liu and Gaiyun Shen, and my sister, Zhijuan Liu for their love and support. 


\section{ACKNOWLEDGEMENTS}

First of all, I would like to express my most sincere thanks to my graduate advisor, Dr. Jian Zuo for his guidance and full support throughout my 5 years Ph.D. studies. Dr. Zuo has put great efforts to train me how to read and criticize published literatures, develop my own ideas and experimental designs to answer the remaining unclear questions relevant to the projects I have been performing. I also thank Dr. Zuo for his advice on how to plan my own future scientific career.

I would also like to express my deep gratitude to other faculty in my graduate committee, Dr. Martine Roussel, Dr. Kristin Hamre, Dr. Michael Dyer, and Dr. Joseph Opferman for their guidance, encouragement and valuable suggestions during each committee meeting. I especially thank the staff scientists in tissue imaging center of St. Jude Children's Research Hospital who helped me a lot in taking the confocal images.

I gratefully acknowledge Ms. Dearman Jennifer, Ms. LingLi Zhang, Dr. Brandon Walters, Dr. Jie Fang, Mr. Thomas Owen, Mr. Mark Crabtree, Dr. Bryan Kuo, Dr. Brandon Cox, Dr. Marcia Mellado Lagarde, Dr. Bradley Walters, Dr. Luigi Iconaru, and Dr. Tetsuji Yamashita in the Zuo Laboratory for their valuable collaborations, inspiring discussion and friendship. In addition, I appreciate that Dr. M. Mishina from Saitama Medical University (Japan) who provided Sox $2^{\text {loxp/loxp }}$ mice; Dr. W. Richardson from University of College London (UK) who provided Fgfr $3^{i C r e E R+}$ mice; Dr. G. Oliver from St. Jude Children's Research Hospital who provided the Prox ${ }^{C r e E R /+}$ mice; Dr. M. Fero from Fred Hutchinson Cancer Research Center who provided $p 27^{\text {loxp/loxp }}$ mice; Dr. J. Robbins from Cincinnati Children's Hospital Medical Center who provided CAG-EGFP+ reporter mice; Dr. J. Johnson from UT Southwestern Medical Center who provided Atohl-EGFP + mice; Dr. Ryoichiro Kageyama from Kyoto University who provided Hes $5^{\text {LacZ/+ }}$ mice; Dr. Sakai from Kyoto Prefectural University of Medicine who provided p2 $7^{\text {Kip } 1}$-luciferase vector and Dr. Kundu from St. Jude Children's Research Hospital who provided the immortalized MEF cells.

I thank my wife Fang Lei; my parents: Chenliang Liu, and Gaiyun Shen, and My sister, Zhijuan Liu for their family support and encouragement.

During the course of my Ph.D. studies, I was supported in part by The Hartwell Individual Biomedical Research Award, a grant from the Office of Naval Research (N000140911014), the American Lebanese Syrian Associated Charities (ALSAC) of St. Jude Children's Research Hospital, and a Travel Award from Academic Programs of St. Jude Children's Research Hospital, University of Tennessee Health Science Center and Society of Developmental Biology. 


\begin{abstract}
Mammalian inner ear cochlear auditory hair cells (HCs) and adjacent supporting cells (SCs) are believed to derive from the same progenitors during development. However, unlike SCs of non-mammalian vertebrates, mammalian cochlear SCs cannot be converted into functional hair cells ( $\mathrm{HCs}$ ) after damage, thus leading to permanent deafness. To entitle mammals with the ability to restore hearing capacity after HC damage, we first achieved proliferation of SCs by acute ablation of p27 or Sox 2 . Secondly, we overactivated Notch 1 signaling in the mouse inner ear at different developmental stages, and found that the ability of Notch signaling in generating new HCs declines after birth. Last, we achieved reprogramming of neonatal and juvenile, but not adult, SCs into HCs, through ectopically expressing Atoh1 in SCs. Taken together, Atoh1 is able to reprogram SCs into HCs, and inactivation of p27 or Sox 2 can achieve proliferation of SCs. This work builds the foundation for future HC regeneration studies. Combining these two manipulations may represent a promising approach to recapitulate the cellular events occurring in the process of $\mathrm{HC}$ regeneration in non-mammalian vertebrates.
\end{abstract}




\section{TABLE OF CONTENTS}

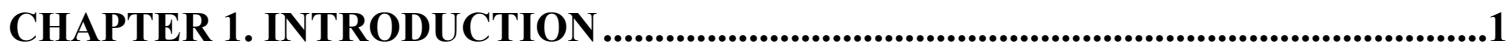

1.1 Similarity between Hair Cell Development and Regeneration...................................

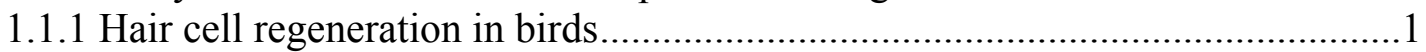

1.1.2 Mechanism of avian hair cell regeneration....................................................

1.2 Key Genes or Signals in Mouse Cochlear Development............................................1

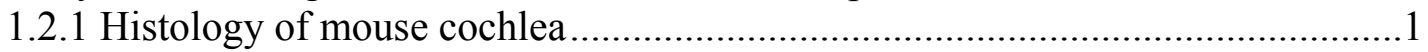

1.2.2 Roles of Notch signaling during mouse cochlear development............................

1.2.3 Roles of Atoh1 in mouse cochlear development .................................................

1.2.4 Roles of Sox2 in mouse cochlear development .................................................

1.2.5 Functions of p27 in mouse cochlear development.............................................5

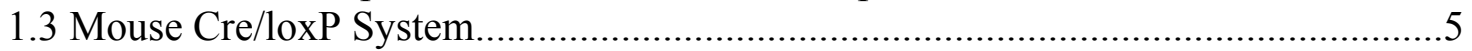

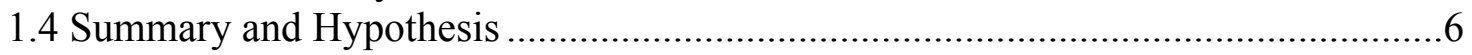

\section{CHAPTER 2. P27 IS REQUIRED TO MAINTAIN QUIESCENCE OF}

NEONATAL PILLAR CELLS......................................................................................7

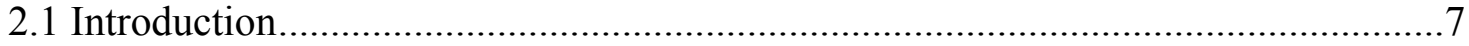

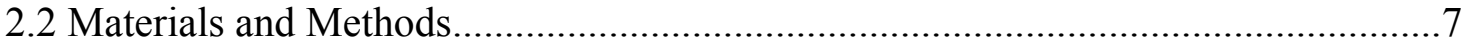

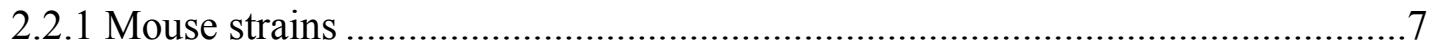

2.2.2 Histology and immunofluorescence ............................................................

2.2.3 Cell proliferation assay ............................................................................

2.2.4 Cell counting and cell death.....................................................................

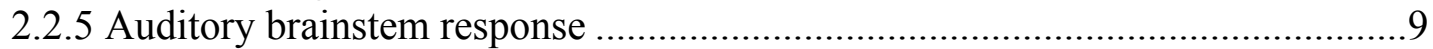

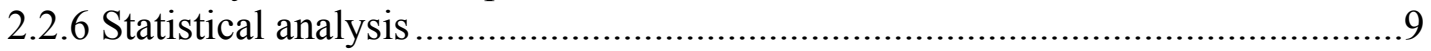

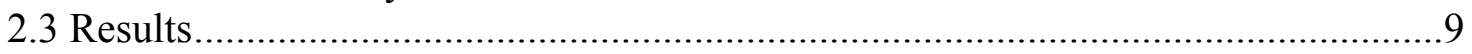

2.3.1 p27-null pillar cells leave quiescence and reenter S phase ……….....................

2.3.2 p27-null pillar cells complete the cell cycle and generate daughter cells.........14

2.3.3 p27-null daughter cells can further proliferate but continue to express

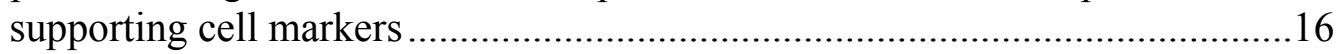

2.3.4 Endogenous hair cells undergo cell death after P15 .......................................16

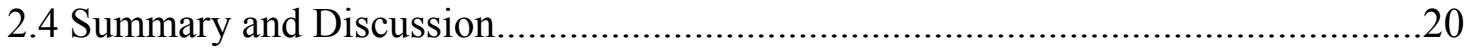

2.4.1 The p27 and cell cycle control in cochlear supporting cells .............................20

2.4.2 Comparison with avian $\mathrm{HC}$ regeneration........................................................22

\section{CHAPTER 3. SOX2 IS REQUIRED TO MAINTAIN QUIESCENCE OF} INNER PILLAR CELLS ..............................................................................................24

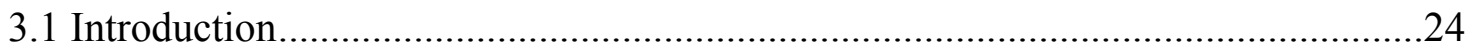

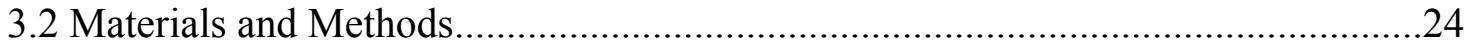

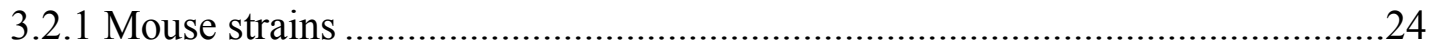

3.2.2 Tissue preparation, immunofluorescence and analysis....................................25

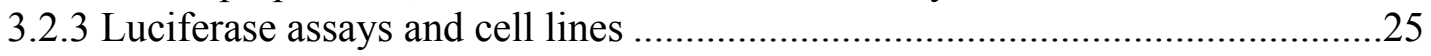

3.2.4 Chromatin immunoprecipitation (ChIP) assay ..............................................26

3.2.5 Auditory brainstem response and statistical analyses .......................................26 


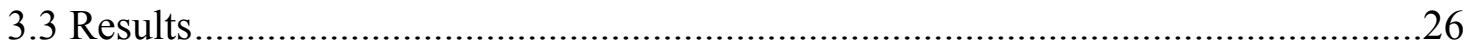

3.3.1 Neonatal inner pillar cells proliferate after acute deletion of Sox 2 .................26

3.3.2 Juvenile inner pillar cells proliferate after acute deletion of Sox2 …...............28

3.3.3 Sox2 deletion in adult inner pillar cells leads to limited proliferation...............30

3.3.4 Functional consequences due to inducible loss of Sox 2 in the mouse

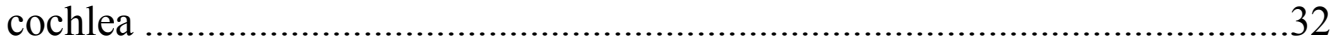

3.3.5 Overexpression of Sox 2 results in activation of the $\mathrm{p} 27$ promoter in vitro ......36

3.3.6 Endogenous levels of Sox 2 bind near the p27 locus.......................................36

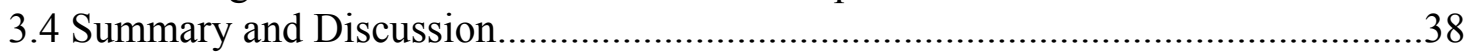

3.4.1 A novel role for Sox 2 in postnatal cochlear supporting cells ............................38

3.4.2 Heterogeneous effects of Sox2 ablation in different supporting cells ...............38

3.4.3 Heterogeneous signals of cell cycle regulation in different supporting cells ...39

3.4.4 Transient modulation of Sox 2 and $\mathrm{p} 27$ for mammalian hair cell

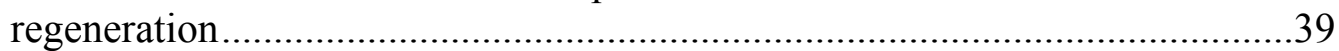

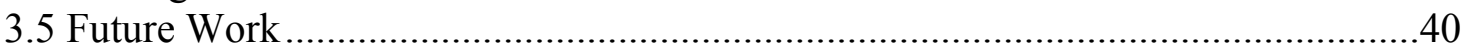

\section{CHAPTER 4. OVERACTIVATION OF NOTCH 1 SIGNALING GENERATES NEW HAIR CELLS AT EARLY EMBRYONIC AGES...............................................42}

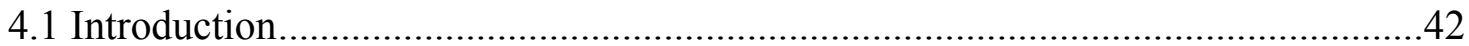

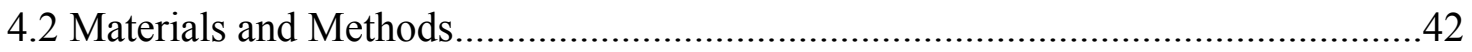

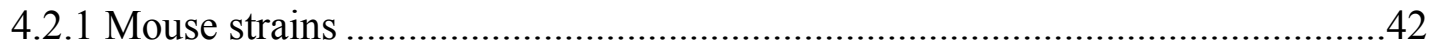

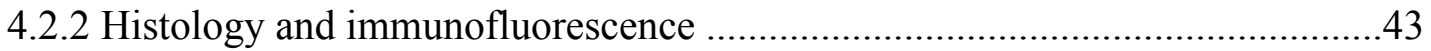

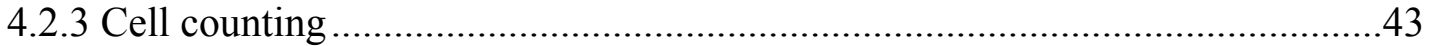

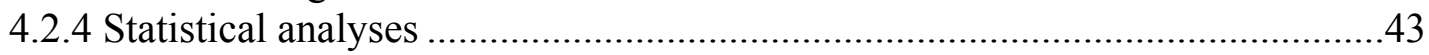

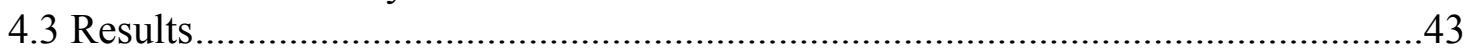

4.3.1 Constitutive overactivation of NICD in otocyst cells generates hair cells .......43

4.3.2 Cellular responsiveness to ectopic NICD declines during mouse cochlear

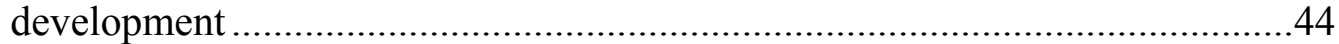

4.3.3 Almost all cochlear progenitors experiencing Notch1 signaling commit to supporting cell fate in normal development....................................................48

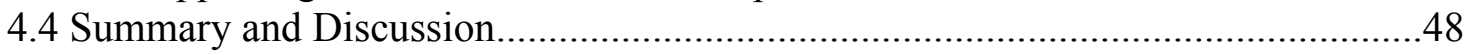

4.4.1 Lateral induction and lateral inhibition effects of Notch1 signaling ................48

4.4.2 Roles of Notch1 signaling in development of the cochlear neuronal lineage...50

4.4.3 Different dosages of Notch1 signaling activity during cochlear

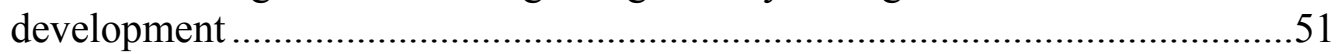

4.4.4 Comparison of three different models explaining the induction of ectopic hair cells in mice ……..............................................................................

4.4.5 Implication of Notch1 signaling in $\mathrm{HC}$ regeneration in mammals ...................52

\section{CHAPTER 5. ECTOPIC ATOH1 REPROGRAMS SUPPORTING CELLS}

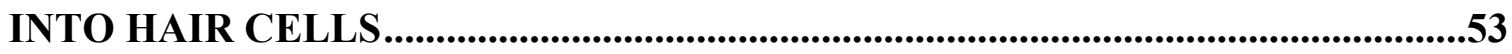

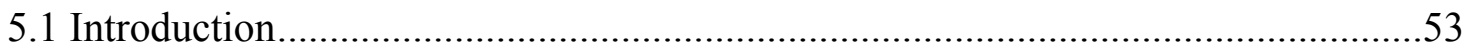

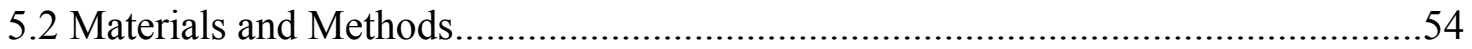

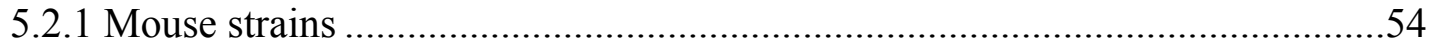

5.2.2 Antibodies and reagents ...........................................................................54

5.2.3 Quantification of newly generated hair cells ...................................................54 


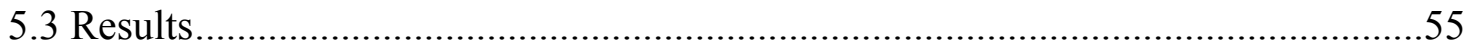

5.3.1 Generation of inducible Atoh1 overexpressing mouse lines .............................55

5.3.2 Neonatal inner phalangeal cells (IPHs) can be reprogrammed into inner

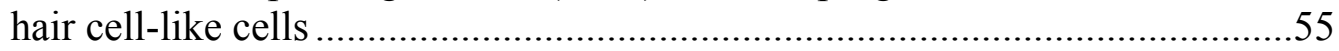

5.3.3 Ectopic Atoh1-HA reprogrammed neonatal pillar and Deiters' cells to hair

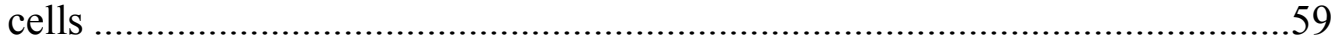

5.3.4 Juvenile pillar and Deiters' cells with ectopic Atoh1-HA also can be reprogrammed to hair cells.........................................................................60

5.3.5 Adult pillar and Deiters' cells are insensitive to ectopic Atoh1-HA expression.

5.3.6 Permanent expression of Atoh1-HA in differentiating endogenous hair

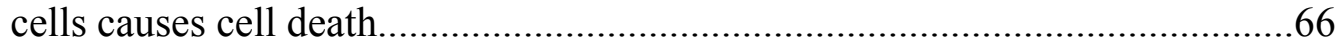

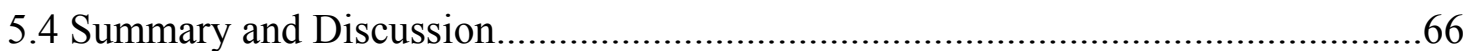

5.4.1 Identifying the newly reprogrammed hair cells at single cell resolution...........68

5.4.2 Atoh1 dosage is a determinant factor for the hair cell fate ...............................68

5.4.3 Diverse reprogramming pathways among different supporting cells ................69

5.4.4 Effects of constitutive Atoh1 expression on hair cell differentiation .................70

5.4.5 Overactivating hair cell fate determining and differentiation genes in supporting cells for hair cell regeneration in mammals ...................................70

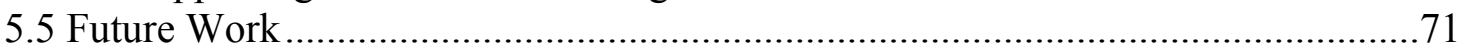

\section{CHAPTER 6. PROSPECTIVE FOR MAMMALIAN HAIR CELL}

REGENERATION...............................................................................................................74

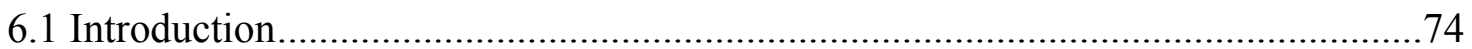

6.2 Conversion of Adult Cochlear Cells into Young and Plastic Ages .........................74

6.3 Application of Mouse Pre-clinical Models to Humans ............................................75

LIST OF REFERENCES.....................................................................................................77

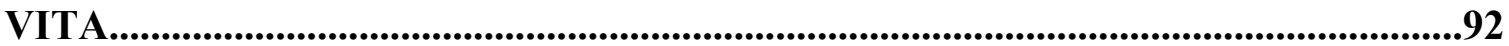




\section{LIST OF FIGURES}

Figure 1-1 Histology of the mouse auditory epithelium, organ of Corti.....................2

Figure 2-1 p27 is progressively deleted after tamoxifen induction..........................11

Figure 2-2 Cell cycle reentry of p27-null PCs.................................................... 12

Figure 2-3 p27-null PCs proliferation peaks at P4 in the apical turn. .......................13

Figure 2-4 p27-null PCs progress through the M phase and generate daughter cells.

Figure 2-5 Daughter cells further proliferate.

Figure 2-6 Daughter cells migrate upward into the HC layer or the surface of auditory epithelium.

Figure 2-7 Proliferating PCs still express SC markers.

Figure 2-8 $\mathrm{HC}$ death and hearing loss in p27 CKO mice.

Figure 3-1 High iCre activity of $\mathrm{Fgfr} 3^{\mathrm{iCreER}+}$ mice.

Figure 3-2 Deletion of Sox2 leads to proliferation of neonatal inner pillar cells (IPCs).

Figure 3-3 Sox2 ablation leads to proliferation of juvenile inner pillar cells.

Figure 3-4 Limited proliferation of inner pillar cells (IPCs) occurs after Sox 2 is ablated at adult age.

Figure 3-5 Limited proliferation of pillar cells (PCs) occurs after p27 is ablated at adult age.

Figure 3-6 Long term effects caused by proliferation of neonatal inner pillar cells (IPCs).

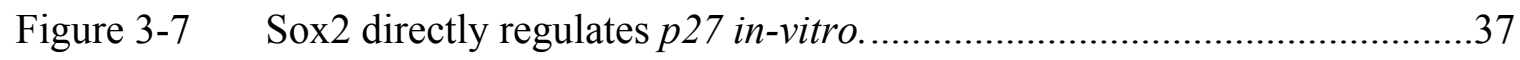

Figure 3-8 Hes5 expression pattern in wild type and Notch mutant mice..................41

Figure 4-1 Ectopic HCs in the cochlea with constitutive Notch1 signaling...............45

Figure 4-2 Expression pattern of Sox10 in ectopic cochlear SCs. ...........................46

Figure 4-3 Declined response of older embryonic cochlear cells to ectopic NICD expression. 
Figure 4-4 Genetic fate mapping analysis of cells experiencing Notch1 signaling. ...49

Figure 5-1 Atoh1 reprogrammed neonatal inner phalangeal cells (IPHs) to hair

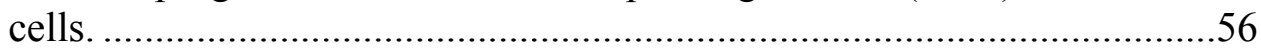

Figure 5-2 Characterization of Cre activity in $P l p^{\text {CreER+ }}$ mouse. ..................................58

Figure 5-3 Reprogramming of neonatal PCs and DCs into HCs by ectopic expression of Atoh1-HA.

Figure 5-4 Ectopic Atoh1-HA reprogrammed juvenile PCs and DCs into HCs.........63

Figure 5-5 Endogenous Atoh1 gene in new HCs is reactivated.................................64

Figure 5-6 Adult PCs and DCs were insensitive to the ectopic Atoh1-HA..................65

Figure 5-7 Effects of permanent Atoh1 expression in endogenous differentiating

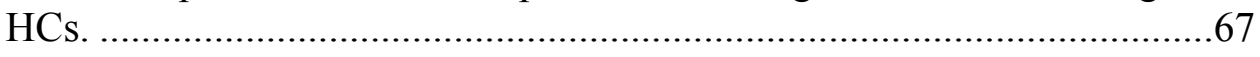

Figure 5-8 Characterization of recombinase Cre activity in Otoferlin $^{\mathrm{Cre} / \mathrm{+}}$ mice..........72

Figure 5-9 Experimental strategy for determining the functionality of the new hair cells deriving from inner phalangeal cells. ................................................73

Figure 6-1 Screening drugs for translational study in human $\mathrm{HC}$ regeneration...........76 


\section{LIST OF ABBREVIATIONS}

$\begin{array}{ll}\text { ABR } & \text { Auditory Brainstem Response } \\ \text { BP } & \text { Basilar Papilla } \\ \text { BrdU } & \text { 5-Bromo-2'-Deoxyuridine } \\ \text { CtBP2 } & \text { C-terminal-Binding Protein 2 } \\ \text { DCs } & \text { Deiters' Cells } \\ \text { EdU } & \text { 5-Ethynyl-2'-Deoxyuridine } \\ \text { FISH } & \text { Fluorescence In Situ Hybridization } \\ \text { GER } & \text { Greater Epithelium Ridge } \\ \text { HCs } & \text { Hair Cells } \\ \text { HEK } & \text { Human Embryonic Kidney 293 cells } \\ \text { IHCs } & \text { Inner Hair Cells } \\ \text { IPCs } & \text { Inner Pillar Cells } \\ \text { IPHs } & \text { Inner Phalangeal Cells } \\ \text { LER } & \text { Lesser Epithelium Ridge } \\ \text { MEF } & \text { Mouse Embryonic Fibroblasts } \\ \text { NICD } & \text { Notch1 Intracellular Domain } \\ \text { OHCs } & \text { Outer Hair Cells } \\ \text { OPCs } & \text { Outer Pillar Cells } \\ \text { PCs } & \text { Pillar Cells } \\ \text { P19 } & \text { P19 } 9 \text { Ink4d } \\ \text { P21 } & \text { P21 Cip1 } \\ \text { P27 } & \text { P27 Kip1 } \\ \text { SCs } & \text { Supporting Cells } \\ \text { SGNs } & \text { Sprial Ganglion Neurons } \\ \text { TUNEL } & \text { Terminal Deoxynucleotidyl Transferase dUTP Nick End Labeling } \\ & \end{array}$




\section{CHAPTER 1. INTRODUCTION}

\subsection{Similarity between Hair Cell Development and Regeneration}

\subsubsection{Hair cell regeneration in birds}

The capacity of birds to regenerate auditory hair cells (HCs) from the adjacent supporting cells (SCs) was identified about 20 years ago. ${ }^{1}$ Since that discovery, other non-mammalian vertebrates such as fish and amphibians were also found to have $\mathrm{HC}$ regeneration ability. Briefly, two days after either noise or aminoglycoside antibiotic (i.e. genetamicin)-induced HC damage to the birds' auditory organ, also referred as to basilar papilla (BP), the nascent embryonic-like HCs appear in the BP region where HC loss occurs. The nascent HCs also become fully differentiated and are functional within 2 4 weeks when the normal histological pattern is restored and hearing is recovered., ${ }^{2,3}$

\subsubsection{Mechanism of avian hair cell regeneration}

There are two proposed molecular mechanisms underlying the $\mathrm{HC}$ regeneration process in the birds' BP. The first is direct transdifferentiation in which upon HC damage, the neighboring SCs rapidly switch to a $\mathrm{HC}$ fate and undergo differentiation to HCs without cell division, which contributes to the first regenerated HC pools. ${ }^{4}$ The second is mitotic regeneration which occurs relatively later than the direct transdifferentiation. It postulates that SCs first divide and produce daughters which further develop into either the HC or SC fate. ${ }^{5,6}$ Both direct transdifferentiation and mitotic regeneration are necessary to restore the normal morphology of the birds' BP. Briefly; the newly regenerated HCs recapitulate the pathway of the normal chick HC development. ${ }^{6}$ These models highlight the promise of manipulating the key developmental genes to endow mammals with the capacity to regenerate HCs after damage occurs, which normally does not occur.

\subsection{Key Genes or Signals in Mouse Cochlear Development}

\subsubsection{Histology of mouse cochlea}

The mouse cochlea is derived from the ventral part of the otocyst adjacent to the hindbrain. The primordial cochlea first appears around embryonic day 11 (E11) and finally transforms into a coiled organ with 1.75 turns before birth. The auditory epithelium, also referred as organ of Corti, contains two main cell types, hair cells (HCs) and supporting cells (SCs) (Figure 1-1). Outside the organ of Corti lies the two non-sensory regions, greater epithelium ridge (GER) in the medial side and lesser epithelium ridge (LER) in the lateral side. HCs and SCs are believed to derive from the 


\section{Myosin-VI+ Sox $2+$}

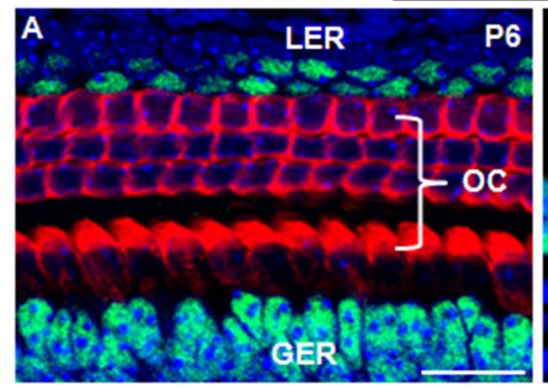

Whole mount

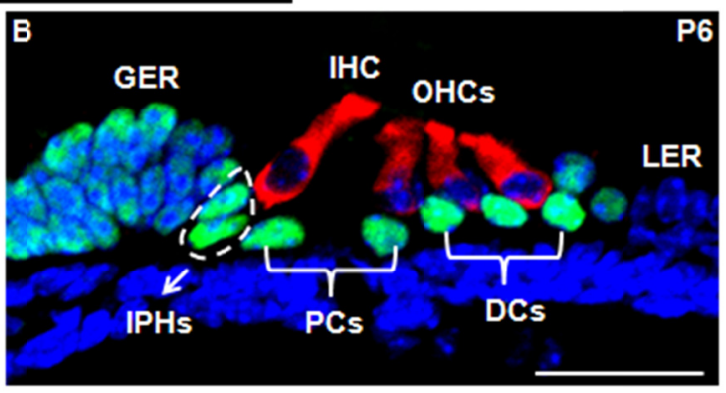

Trans-section

Figure 1-1 Histology of the mouse auditory epithelium, organ of Corti.

(A) Whole mount image of the organ of Corti (OC). Inside OC lie three rows of outer hair cells (OHCs) and one row of inner hair cells (IHCs). Both OHCs and IHCs are visualized in red with an antibody to Myosin-VI (a hair cell specific marker). Lateral and medial to OC are the LER (lesser epithelium ridge) and GER (greater epithelium ridge). While OC is defined as cochlear sensory regions, LER and GER are defined as the non-sensory areas. (B) In each trans-section image of OC, HCs are sitting on the top of the supporting cells (SCs). There are at least three subtypes of SCs, from medial to lateral, which are defined as inner phalangeal cells (IPHs), pillar cells (PCs) and Deiters' cells (DCs). These names are given primarily by their morphology and location. The detailed molecular differences among these different SC subtypes remains poorly understood. These SCs are labeled with the postnatal SC marker Sox2 in green. Blue is Hoechst staining DNA. 
prosensory progenitors in the sensory regions. ${ }^{7}$ There is one row of inner hair cells (IHCs) and three rows of outer hair cells (OHCs). IHCs and OHCs work differently to detect sound. The IHCs are the primary mechanosensory cells that are connected to $\sim 90 \%$ of the cochlear spiral ganglion neurons. The OHCs mainly are the amplifier of the coming sound and are innervated by $\sim 10 \%$ of the spiral ganglion neurons (SGNs) which further replay the hearing information to auditory cortex.

\subsubsection{Roles of Notch signaling during mouse cochlear development}

The Notch signaling pathway in metazoans regulates the short-range communication between cells, such as cell death, proliferation and cell fate specification. ${ }^{8}$ In mammals, there are four different Notch receptors (Notch1 to Notch4) and different Notch ligands (i.e. Jagged1, Jagged2, D111 and D113). ${ }^{9-12}$ Gain and loss of function studies suggest that Notch 1 is the primary receptor expressed in mouse cochlea. ${ }^{13-18}$ Currently Notch1 signaling is assumed to incorporate all Notch signaling activities in mouse cochlea. When the ligands bind to Notch1 which then is cleaved, allowing the intracellular domain to translocate into the nucleus, where it interacts with Rbp-J, and induces activation of different Notch target genes.

Notch1 signaling generally elicits dual but contrasting effects: the first is the lateral induction effect. ${ }^{19-22}$ The lateral induction effect occurs in the developmental period when cochlear cells are undergoing rapid proliferation. Among the cochlear cells, those with active Notch1 signaling are specified as cochlear progenitors, although the detailed mechanism is poorly characterized. After the cochlear progenitor cell pool is generated, progenitors will undergo cell fate specification and differentiate into either HCs or SCs, mediated by the second lateral inhibition effect of Notch1 signaling. ${ }^{23-29}$ Briefly, cells maintaining Notch1 signaling activity develop into SCs, and cells gradually losing Notch1 signaling activity become HCs.

Because Notch1 signaling specify the progenitor cells, overactivation of Notch1 signaling in very early embryonic mouse inner ear non-sensory regions recently is shown to generate ectopic HCs. ${ }^{13,15}$ This provides us with a promising approach to generate new HCs after HC damage in mammals. However, it remains elusive whether such an effect still can be elicited when Notch1 signaling is overactivated in neonatal or adult mouse cochlea. This question is of particular interest in studies of mammalian HC regeneration because the majority of the human deafness occurs at postnatal ages from various ototoxic factors. Thus, clarifying this question is one of the three goals in my graduate dissertation.

\subsubsection{Roles of Atoh1 in mouse cochlear development}

Atoh1, atonal homolog 1 in Drosophila or Math1 in mice, is a basic helix-loop-helix (bHLH) transcription factor. We will use Atoh1 hereafter throughout the dissertation. It is a proneural gene required in the formation of chordotonal organs and 
photoreceptors in Drosophila. ${ }^{30,31}$ In mammals, Atoh1 is expressed in the cerebellum, the dorsal neural tube, the intestine, and the inner ear. ${ }^{32-35}$

During mouse cochlear development, Atoh1 is first expressed around $\sim \mathrm{E} 12$ in the common progenitors for HCs and SCs. ${ }^{36,37}$ Atoh1 is necessary for specifying the HC progenitors, and Atohl germ line knockout mice lack HCs in the cochlea. ${ }^{38} \mathrm{An}$ antagonistic interaction between Notch1 signaling and Atoh1 has been proposed. Briefly, Notch1 signaling can inhibit the transcriptional activity of Atoh1. Overactivation of Notch1 signaling in developmental stages $(\sim \mathrm{E} 13)$, when cochlear progenitors are about to choose their final fate, blocks further formation of HCs. At postnatal day 0 (P0), expression of Atoh1 is restricted into HCs and becomes undetectable in SCs. ${ }^{39,40}$ Furthermore, Atoh1 expression in HCs is down-regulated dramatically within the first postnatal week and becomes undetectable in HCs in P6. ${ }^{39,40}$

Although the molecular mechanism remains unclear, overexpression of Atoh1 can induce ectopic HCs in mouse cochlea in either in vivo or in vitro conditions. ${ }^{40-44}$ These data support that Atoh1 is sufficient in specifying the HC fate. However, it remains unknown whether Atoh1 is sufficient to guide the new HCs to differentiate normally. This is one of the key questions to be answered in my dissertation.

\subsubsection{Roles of Sox2 in mouse cochlear development}

Sox2 is a transcription factor that carries a DNA-binding high-mobility group (HMG) domain and affects gene transcription through collaboration with different partners that are specific to cell type or age. ${ }^{45}$ The embryonic development of the cochlea is tightly dependent on Sox $2 .{ }^{46-48}$ Sox 2 expression starts at the onset of otocyst development and is gradually restricted to different sensory regions of the inner ear. ${ }^{49}$ In the cochlear portion, Sox 2 is first expressed in progenitors, and later on its expression is gradually decreased and becomes undetectable in $\mathrm{HCs}$, while its expression is maintained in SCs. ${ }^{50,51}$

The exact roles of Sox 2 are developmental stage-and cell-context dependent in different tissues. ${ }^{52-55}$ In mouse cochlea, the roles of Sox2 between E9 and E13.5 remain poorly understood. Overactivation of Sox 2 in cochlear progenitor cells ( E13) inhibits $\mathrm{HC}$ formation, suggesting the existence of antagonistic interactions between Sox 2 and Atoh1 ${ }^{48}$ In addition, Prox 1 is a target gene of Sox 2 and the initial expression of Prox 1 is Sox2-depedent, as supported by gain and loss-of-function studies in vitro. ${ }^{48}$ Although Sox2 is expressed in postnatal SCs (both neonatal and adult ages), its role remains unknown. We are interested in determining whether Sox 2 is a key determinant factor to maintain the SC fate and whether the antagonistic interactions (at embryonic ages) between Sox2 and Atoh1 remain at postnatal ages. Clarifying this issue is of particular interest for both normal cochlear development and $\mathrm{HC}$ regeneration study. It is another goal of my graduate dissertation. 


\subsubsection{Functions of p27 in mouse cochlear development}

$\mathrm{P} 27^{\mathrm{Kip} 1}$ is a Cip/Kip family cell cycle inhibitory protein. It is also referred as to Cdkn1b. We will use $\mathrm{p} 27$ throughout the dissertation to define $\mathrm{p} 27^{\mathrm{Kip} 1}$. It primarily functions to block cyclin-dependent kinases and block cell cycle progression. ${ }^{56} p 27^{-/}$ germ line knockout mice are larger than their wild type littermates. ${ }^{57-59}$ Recently, p27 was also shown to regulate cell migration and promote tumorigenesis under specific cellular conditions. ${ }^{60,61}$

During the cochlear development, the primary role of p27 is to drive progenitor cells to precisely exit the cell cycle. The wave of turning on p27 initiates at the apical turns around E12.5 and migrates to the basal turns around E14.5. ${ }^{62}$ Following cell cycle exit, these progenitors start differentiating in an opposite gradient, from basal to apical turns. ${ }^{63}$ As expected, cochlear progenitor cells in the $p 27^{-/}$germ line knockout mice cannot exit the cell cycle at the correct time and undergo prolonged proliferation, resulting in an expanded progenitor cell pool which gives rise to supernumerary HCs and SCs. ${ }^{64,65}$

When isolated neonatal mouse cochlear SCs are cultured in vitro, p27 expression is decreased and SCs proliferate and switch to HCs. ${ }^{66}$ However, when neonatal cochlear explants are cultured and transfected with vectors expressing $p 27$ shRNA, SCs in LER regions can proliferate but maintain their intrinsic SC fate. It remains unknown what phenotype the SCs inside the organ of Corti will adopt when $p 27$ is deleted. This question will be answered in my graduate dissertation.

\subsection{Mouse Cre/loxP System}

The powerful mouse genetic Cre/loxP system enables conditional gain or loss-offunction studies. ${ }^{67}$ The Cre-loxP mechanism was first discovered in P1 bacteriophage. ${ }^{68,69}$ $\mathrm{Cre}$ is a recombinase that catalyzes recombination between two loxP sites. In a Cre expressing mouse knock-in or transgenic strain, Cre is controlled by a ubiquitously active (i.e. CAG or Rosa 26) or tissue specific gene promoter (i.e. Proxl or Fgfr3 in cochlear $\mathrm{SCs})$. To activate the recombinase at different stages, Cre can be fused to the mutated ligand-binding domain (LBD) of the estrogen receptor (ER). In the absence of tamoxifen, CreER is sequestered in the cytoplasm by the heat shock protein 90 (Hsp90). ${ }^{70}$ However, when tamoxifen is available and binds with the LBD, CreER undergoes a conformational change and is released from Hsp90, leading to the translocation of CreER into the nucleus. Depending on the orientation and location of the loxP sites, Cre-mediated recombination can lead to three different outcomes: inversion, translocation or deletion. ${ }^{71}$ Currently, the majority of Cre-loxP systems are used to create a deletion effect for which the two loxP sites are designed in the same location and orientation. However, depending on the sequence that is deleted in each mutant mouse model, either gain or loss-of-function effects can be elicited. 


\subsection{Summary and Hypothesis}

The goal of my graduate dissertation is to provide information for future mammal $\mathrm{HC}$ regeneration. Due to the similarity between avian $\mathrm{HC}$ regeneration and normal $\mathrm{HC}$ development, the key rationale of our strategy for mammal $\mathrm{HC}$ regeneration is to reactivate the key $\mathrm{HC}$ developmental genes in SCs which are then expected to follow the $\mathrm{HC}$ differentiation track. We propose three hypotheses:

The first is that isolated cochlear SCs can downregulate $\mathrm{p} 27$, proliferate and become HCs in vitro. My first hypothesis is that acute inactivation of $\mathrm{p} 27$ in postnatal mouse cochlear SCs will promote their proliferation and conversion into HCs.

The second is that Atoh1 expression will be derepressed upon acute ablation of Sox 2 and SCs will be converted into HCs. We proposed this hypothesis because Sox 2 antagonizes Atoh1 during the embryonic mouse cochlear development. Sox 2 will be acutely deleted in mouse cochlear SCs at different ages in vivo.

The third is that Atoh1 can reprogram postnatal SCs to adopt the $\mathrm{HC}$ fate. We aimed to achieve direct transdifferentiation from SCs to HCs, similar to what occurs in avian SCs when HCs are damaged. Atoh1 will be ectopically induced in the postnatal mouse cochlear SCs at different ages in vivo. 


\section{CHAPTER 2. P27 IS REQUIRED TO MAINTAIN QUIESCENCE OF NEONATAL PILLAR CELLS}

\subsection{Introduction}

When isolated mouse neonatal SCs are cultured in vitro, they can dowregulate p27, proliferate and turn on hair cell specific genes, indicating the occurrence of cell fate conversion. ${ }^{66}$ However, whether similar cellular events will occur in the in vivo environment remains unclear. Because the p27 germ line knockout mice have defective cochlear development during embryonic ages, ${ }^{64,65}$ the neonatal SCs are not comparable to those of the wild type littermates. To bypass defective embryonic development, we took advantage of p27 conditional knockout mice.

To acutely ablate $\mathrm{p} 27$ at different postnatal ages, we requested the $p 27^{\text {loxp/loxp }}$ mice $^{72}$ from the laboratory of Dr. Matthew Fero at the Fred Hutchinson Cancer Research Center, and further characterized two different CreER mouse lines Prox $1^{\mathrm{CreER} /+}$ and Fgfr $3^{\text {iCreER+ }}{ }^{73-75}$ Prox $1^{\text {CreER/+ }} ; p 27^{\text {loxp/loxp }}$ mice were treated with tamoxifen at P0 and P1 to delete $p 27$ at neonatal ages, while $\mathrm{Fgfr} 3^{\text {iCreER+ }} ; p 27^{\text {loxp/loxp }}$ mice were treated with tamoxifen at P6 and P7, or adult ages (P30) to ablate $p 27$ at juvenile and adult ages. By analyzing these genetic mouse models, we will determine whether $p 27$-null SCs proliferate and/or become $\mathrm{HCs}$, as it is the case in vitro. ${ }^{66}$

\subsection{Materials and Methods}

\subsubsection{Mouse strains}

Prox $1^{\text {CreER/+ }}$ and $p 27^{\text {loxp/loxp }}$ mice were kindly provided by Dr. Guillermo Oliver and Dr. Matthew Fero and described previously. ${ }^{72,76}$ Rosa26-EYFP ${ }^{\text {loxp } /+}$ were purchased from Jackson Laboratory (USA). All animal work conducted during the course of this study was approved by the Institutional Animal Care and Use Committee at St. Jude Children's Research Hospital and performed according to NIH guidelines.

\subsubsection{Histology and immunofluorescence}

Whole embryos at various ages were immersed in 4\% paraformaldehyde (PFA) overnight at $4{ }^{\circ} \mathrm{C}$, after which the whole inner ear was carefully dissected out. For whole mount analysis, the whole cochlear duct and corresponding medial spiral ganglion tissues were divided into three parts, the basal, middle, and apical turns.

Both whole mounts and trans-sections were permeabilized at room temperature for 1 hour in a solution containing 1\% bovine serum albumin and 1\% Triton X-100 in 10 $\mathrm{mM}$ phosphate-buffered saline (PBS, $\mathrm{pH} 7.4$ ). Tissues were then incubated with primary 
antibodies in blocking solution (1\% bovine serum albumin and $0.1 \%$ Triton $\mathrm{X}-100$ in 10 $\mathrm{mM}$ PBS) overnight at $4^{\circ} \mathrm{C}$, followed by 3 washes for 10 minutes each in $10 \mathrm{mM}$ PBS. Then, tissues were incubated with secondary antibodies in the same blocking solution overnight at $4^{\circ} \mathrm{C}$ followed by 3 washes for 10 minutes each in $10 \mathrm{mM}$ PBS. Tissues were then incubated for 30 minutes at room temperature in Hoechst 33342 in 10 mM PBS (Invitrogen, H3570, 1:1,000), followed by 3 washes for 10 minutes each in $10 \mathrm{mM}$ PBS, and finally were mounted in ProLong Gold antifade reagent (Invitrogen, P36934). Samples were dried at room temperature for at least 24 hours.

The following primary antibodies were used: anti-myosin-VIIa (rabbit, 1:200, Proteus Bioscience, 25-6790) or Alexa Fluor 594-conjugated anti-myosin-VIIa (rabbit, 1:30, Proteus Bioscience, specific request), anti-myosin-VI (rabbit, 1:200, Proteus Bioscience, 25-6791) or Alexa Fluor 647-conjugated anti-myosin VI (rabbit, 1:50, Proteus Bioscience, specific request), anti-BrdU (mouse, 1:50, invitrogen, B35131), anti-p27(mouse, 1:150, BD Biosciences, 610242), anti-Prox1 (rabbit, Millipore, 1:500, AB5475), anti-GFP (rabbit, 1:50, Invitrogen, A-21311) or anti-GFP (chicken, 1:1000, Abcam, ab13970), Alexa Fluor 488-conjugated anti-PH3 (rabbit, 1:20, Cell Signaling, 9708), anti-Sox2 (rabbit, 1:1,000, Millipore, AB5603). We used secondary goat anti-rabbit Alexa Fluor 350, 488, 594, and 647 antibodies (1:1,000, Invitrogen, A-21068/11036/11037/21245) and goat anti-chicken Alexa Fluor 488 (1:1,000, Invitrogen, A-11039). For TSA staining, we used goat anti-mouse HRP conjugated secondary antibody (1:100, T20912, Invitrogen).

\subsubsection{Cell proliferation assay}

Mice were given one injection of 5-ethynyl-2'-deoxyuridine (EdU, $20 \mu \mathrm{g}$, Invitrogen, C10083) and were sacrificed 4 hours later or BrdU (50 mg/kg body weight) once every 2 hours for a total of 5 times and were sacrificed 2 hours after the last injection. After cardiac perfusion with $10 \mathrm{mM}$ PBS and 4\% PFA, tissues for BrdU staining were first pretreated with $2 \mathrm{~N} \mathrm{HCl}$ for $25 \mathrm{~min}$ at $37^{\circ} \mathrm{C}$, followed by three 5 minutes washes in $0.1 \mathrm{M}$ Tris- $\mathrm{HCl}(\mathrm{pH} \mathrm{8.0)}$ ) for neutralization and immunostained as described above using an Alexa Fluor 488 or 594 conjugated anti-BrdU antibody (mouse, 1:20, Invitrogen, A-21303 or 21304).

For EdU staining, we followed a protocol recommended by Invitrogen. For BrdU and EdU double staining, mice were injected with BrdU 5 times every two hours at P2, followed by EdU injection 48 hours later (P4) and sacrificed 4 hours after EdU injection. Cochleae were dissected and immersed directly in methanol/acetic acid (3:1) fixative for 6 hours at $4^{\circ} \mathrm{C}$, followed by BrdU immunostaining (BrdU antibody 1:100 dilution) and EdU staining. For BrdU and FISH double staining, we used the same fixation procedure with methanol/acetic acid and anti-BrdU antibody (1:100) were used. The BrdU antibody does not recognize the BrdU antigen, even though BrdU and EdU share the similar structural backbone. 


\subsubsection{Cell counting and cell death}

To account for variability of cell density between different turns of the cochlea, the location of each turn was approximated based on the length of the entire cochlear duct. We quantified cell numbers in $200 \mu \mathrm{m}$ length of the apical (75\% of the length of the cochlear duct, from the hook), middle (50\%) and basal (25\%) turns using a Zeiss LSM 510 confocal microscope. A similar approach was used to calculate the percentage of p27-null PCs and DCs. To quantify PC and DC number at different ages, DCs, PCs and HCs were counted based on location using myosin-VIIa staining to mark HCs. To confirm this method, we also counted DCs, PCs and HCs based on location using Sox 2 staining to label SCs. PC and DC numbers were then normalized to HC numbers. SC/HC ratios calculated by both methods gave similar results and were thus grouped together.

For cell death measurements, Terminal deoxynucleotidyl transferase dUTP nick end labeling (TUNEL) staining was performed according to the protocol provided by the Roche Applied Science, 11684795910/12156792910). Prior to TUNEL staining, myosin VIIa staining was used for cell type determination.

\subsubsection{Auditory brainstem response}

Detailed procedures were described previously. ${ }^{77}$ Briefly, experimental and control littermates mice were intraperitoneally anesthetized with Avertin $(500 \mathrm{mg} / \mathrm{kg}$ body weight). Pure tone pips of 4, 6, 12, 16, 32, and $44 \mathrm{kHz}$ were generated by using a Tucker Davis Technologies (TDT, Gainsville, FL) work station (System III) running SigGen32 software (TDT). The highest sound is $75 \mathrm{~dB}$ SPL in our current TDT system. At each frequency, the mice were given different sounds gradually from $75 \mathrm{~dB}$ SPL to $0 \mathrm{~dB}$ SPL, and the sound threshold was read when no electrophysiological curve was detected.

\subsubsection{Statistical analysis}

All data were expressed as mean \pm S.E.M. Cell counts were compared using an one-way ANOVA followed by a Student's t test with a Bonferroni correction, and ABR thresholds with a two-way ANOVA followed by a Student's t test with a Bonferroni correction. Statistical analysis was conducted using GraphPad Prism 5.0 Software.

\subsection{Results}

\subsection{1 p27-null pillar cells leave quiescence and reenter $S$ phase}

To acutely inactivate $p 27$ in postnatal PCs and DCs, we treated Proxl ${ }^{\mathrm{CreER} /+}$;

p2 $7^{\text {loxp/loxp }}$ mice (p27 conditional knockout, CKO) and Prox $1^{+/+} ; p 27^{\text {loxp/loxp }}$ (CTRL) with 
tamoxifen at $\mathrm{P} 0$ and $\mathrm{P} 1$. In $\mathrm{p} 27 \mathrm{CKO}$ mice, we found that $\mathrm{p} 27$ was progressively deleted in both PCs and DCs in the apical turns between P2 and P6, which suggests that it takes a few days for endogenous $\mathrm{p} 27$ protein to be degraded and eliminated from the cell after gene deletion (Figure 2-1A-D). There was also a higher percentage of p27-null DCs compared to p27-null PCs at all ages tested, which is consistent with Cre activity detected by the reporter assays. ${ }^{73}$ Additionally, the percentage of apical p27-null PCs and DCs was significantly higher than apical Cre+ PCs and DCs detected with the reporter lines (Figure 2-1E-F), which further supports the idea that reporter lines somehow underestimate Cre activity; however, we cannot exclude that p27 deletion results in non-cell autonomous effects.

We next examined cell cycle reentry of p27-null PCs and DCs using incorporation of 5-bromo-2'-deoxyuridine (BrdU) or its analogue 5-ethynyl-2'-deoxyuridine (EdU) ${ }^{78}$ Surprisingly, of $\sim 130 \mathrm{BrdU}+$ or EdU+ cells analyzed from P2 to P6, all were PCs, and not DCs, based on their location (Figure 2-2A-B). Since the organ of Corti has a precisely organized cell arrangement, we were able to distinguish between PCs and DCs relative to HCs. Nuclei of PCs are located at the bottom of the triangle area between IHCs and the inner most row of OHCs, which correspond to the Tunnel of Corti in the adult cochlea. ${ }^{79}$ DC nuclei are underneath, but slightly lateral to nuclei of their corresponding row of outer HCs.

To confirm the accuracy of our location distinctions are accurate and that cell cycle reentry is exclusive to PCs, we used triple immunostaining with BrdU, p75 ${ }^{\mathrm{NGFR}}$ (a marker expressed in postnatal PCs and Hensen cells but not DCs ${ }^{66}$ and EGFP in cochleae dissected from ProxI $I^{\text {CreER } /+} ; p 27^{\text {loxp/loxp }} ; C A G-E G F P+$ mice. Since $\mathrm{p} 75^{\mathrm{NGFR}}$ is expressed on the surface of PCs and BrdU labels the nucleus, we used EGFP to trace the entire cell boundary of $\mathrm{p} 27^{\mathrm{Kip} 1}$-null cells. This allowed us to accurately determine whether $\mathrm{p} 75^{\mathrm{NGFR}}$ and BrdU staining belong to the same cell. All of the $\sim 25$ BrdU/EGFP double positive cells analyzed were also labeled with $\mathrm{p} 75^{\mathrm{NGFR}}$, again demonstrating that only $\mathrm{p} 27^{\mathrm{Kip} 1}$-null PCs can reenter S phase (Figure 2-2C-F). Additionally, in the $\sim 200 \mu \mathrm{m}$ apical area analyzed, we found several BrdU+ (p2 $7^{\text {Kip1 }}$-null) PCs that were EGFP-negative (arrowhead in Figure 2-2D). This might be due to mosaic expression of the $C M V / \beta$-actin promoter or may indicate that EGFP expression varies depending on cell cycle phase. It is also possible that $\mathrm{p} 27^{\mathrm{Kip} 1}$ deletion results in non-cell-autonomous effects. ${ }^{72}$

We further analyzed $\sim 60$ EdU + PCs from P2 and P4 cochleae and found that all were p2 $7^{\mathrm{Kip} 1}$-null, suggesting that cell cycle reentry is cell autonomous (Figure 2-3A-D). Because cell cycle reentry of p $27^{\text {Kip1 }}$-null PCs in vivo was not synchronized and we only injected EdU once or injected BrdU over a period of 10 hours due to toxicity, we were not able to determine whether every $\mathrm{p} 27^{\mathrm{Kip1}}$-null PC reenters the cell cycle. Quantitative analysis of $\mathrm{BrdU}$ incorporation showed that $\mathrm{PC}$ cell cycle reentry initiated at $\mathrm{P} 2$, peaked at P4 and decreased at P6 (Figure 2-3E-H).

It is quite interesting that while DCs have a higher percentage of Cre active cells based on reporter line studies, we did not detect S phase reentry in DCs from P2 to P6. We did not analyze after P6 because the precise architecture of organ of Corti was 

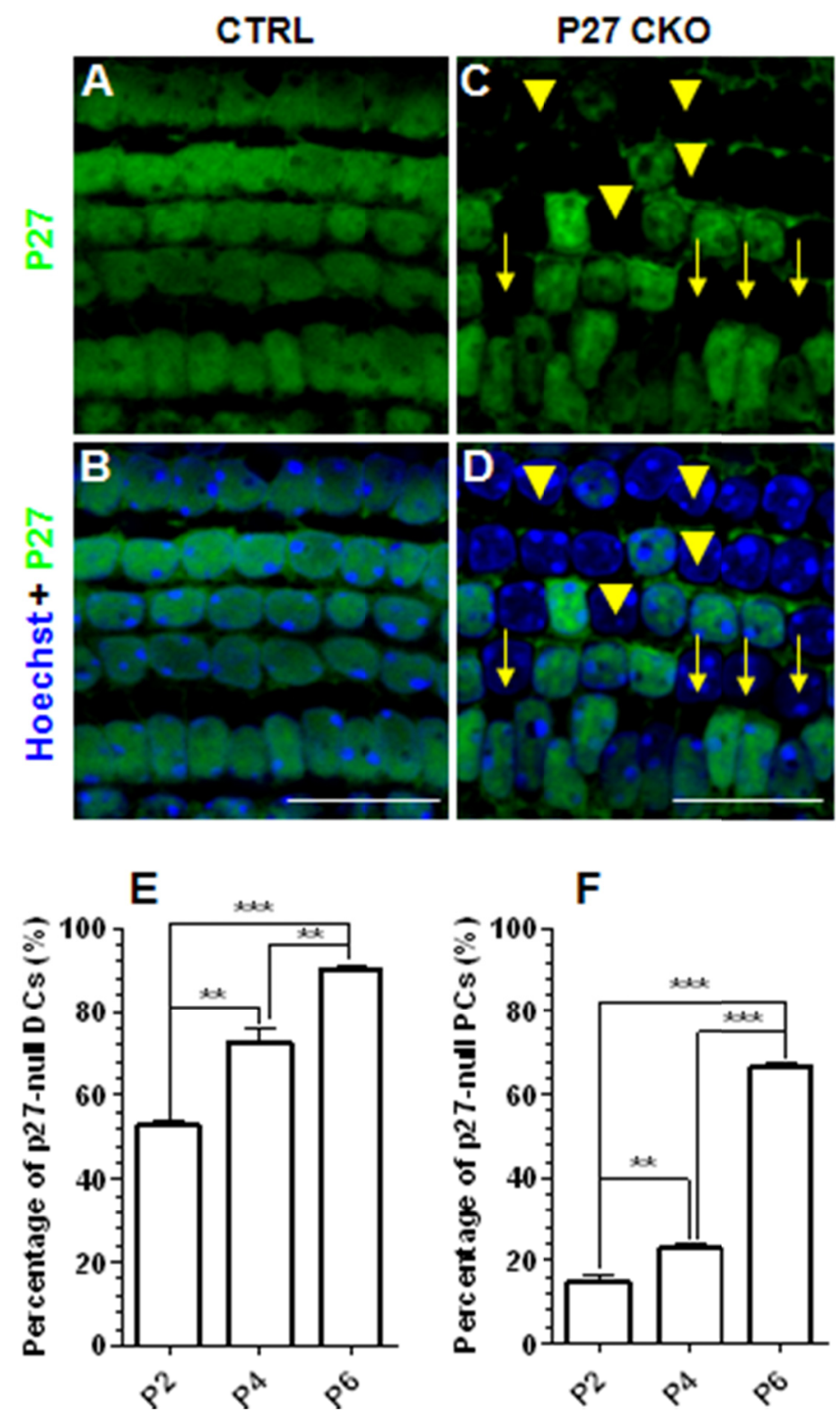

Figure 2-1 p27 is progressively deleted after tamoxifen induction.

(A-D) p27 immunofluorescence of apical cochlear whole mounts in CTRL (A-B) and p27 CKO (C-D) mice at P4. Arrowheads point to p2 $7^{\mathrm{Kip} 1}$-null DCs and arrows identify p27-null PCs. Percentage of apical p27-null DCs (E) and PCs (F) in p27 CKO cochleae at P2, P4 and P6 $(\mathrm{n}=3, * * \mathrm{p}<0.01, * * * \mathrm{p}<0.001)$. Scale bars: $20 \mu \mathrm{m}$. Data is expressed as mean \pm S.E.M. 

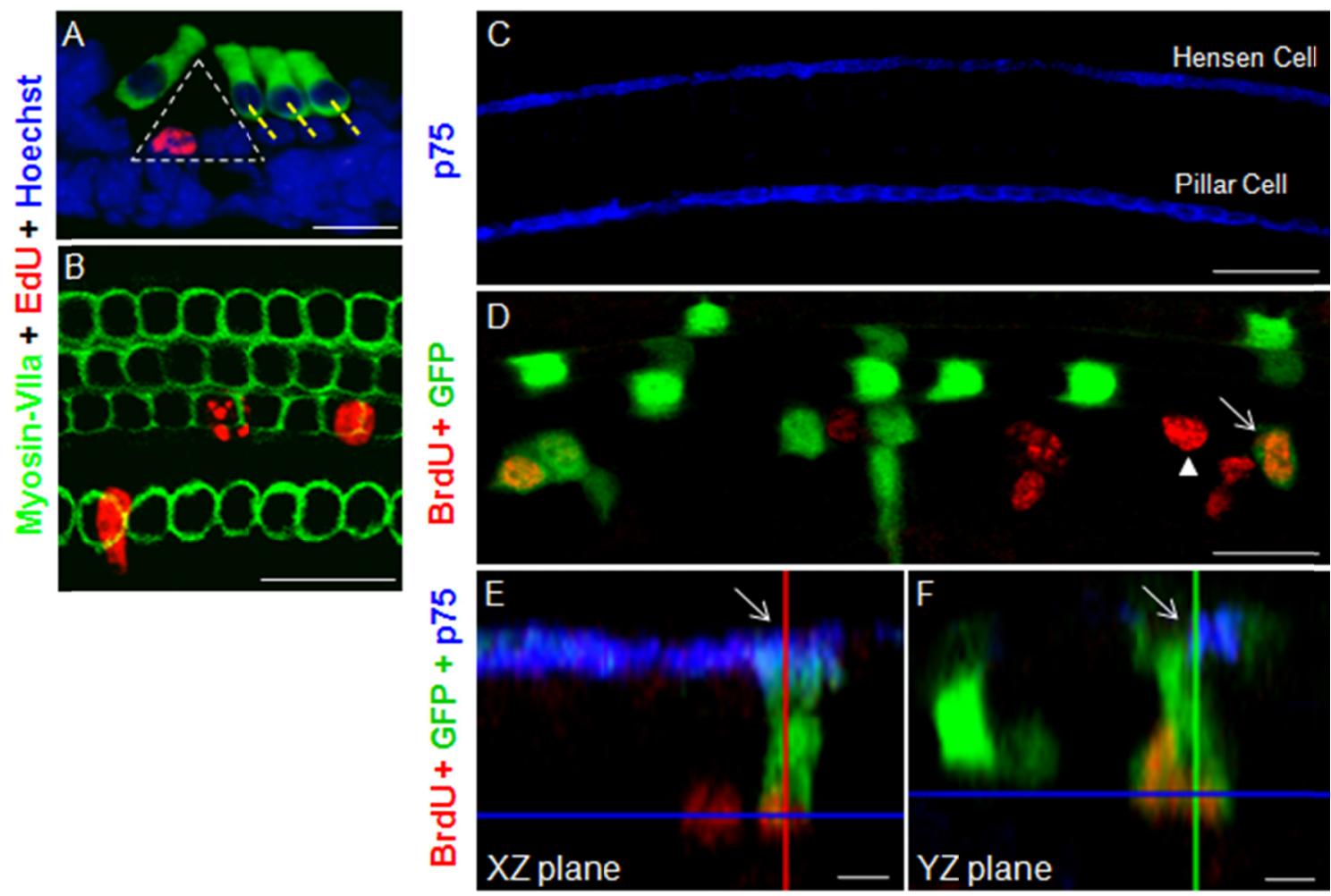

Figure 2-2 Cell cycle reentry of p27-null PCs.

(A) Cross section staining of EdU (red), myosin VIIa (green) and Hoechst 33342 (blue) at P2 in the apical turn of p27 CKO mice. Dashed-line triangle labels the tunnel of Corti and PC nuclei. Yellow dashed lines mark DC nuclei relative to outer HCs. (B) Whole mount confocal projection of EdU (red) and myosinVIIa (green) at P4 in the apical turn of p27 CKO mice. (C-F) Whole mount p $75^{\mathrm{NGFR}}$ staining (blue) at the surface of the auditory epithelium (C), and EGFP (green) and BrdU (red) staining at the SC layer of the auditory epithelium (D). Arrow in D marks a PC that is double positive for EGFP and BrdU. Arrowhead in D identifies a BrdU+, but EGFP-negative PC. Higher magnification view of the arrow labeled $\mathrm{PC}$ at the confocal $\mathrm{XZ}$ plane $(\mathrm{E})$ and confocal $\mathrm{YZ}$ plane $(\mathrm{F})$. This cell is triple stained by $\mathrm{p} 75^{\mathrm{NGFR}}$ (blue) at the surface, BrdU (red) in the nucleus and EGFP (green) in the cytoplasm. Scale bars: A-D is $20 \mu \mathrm{m}$; E and F are $5 \mu \mathrm{m}$. 

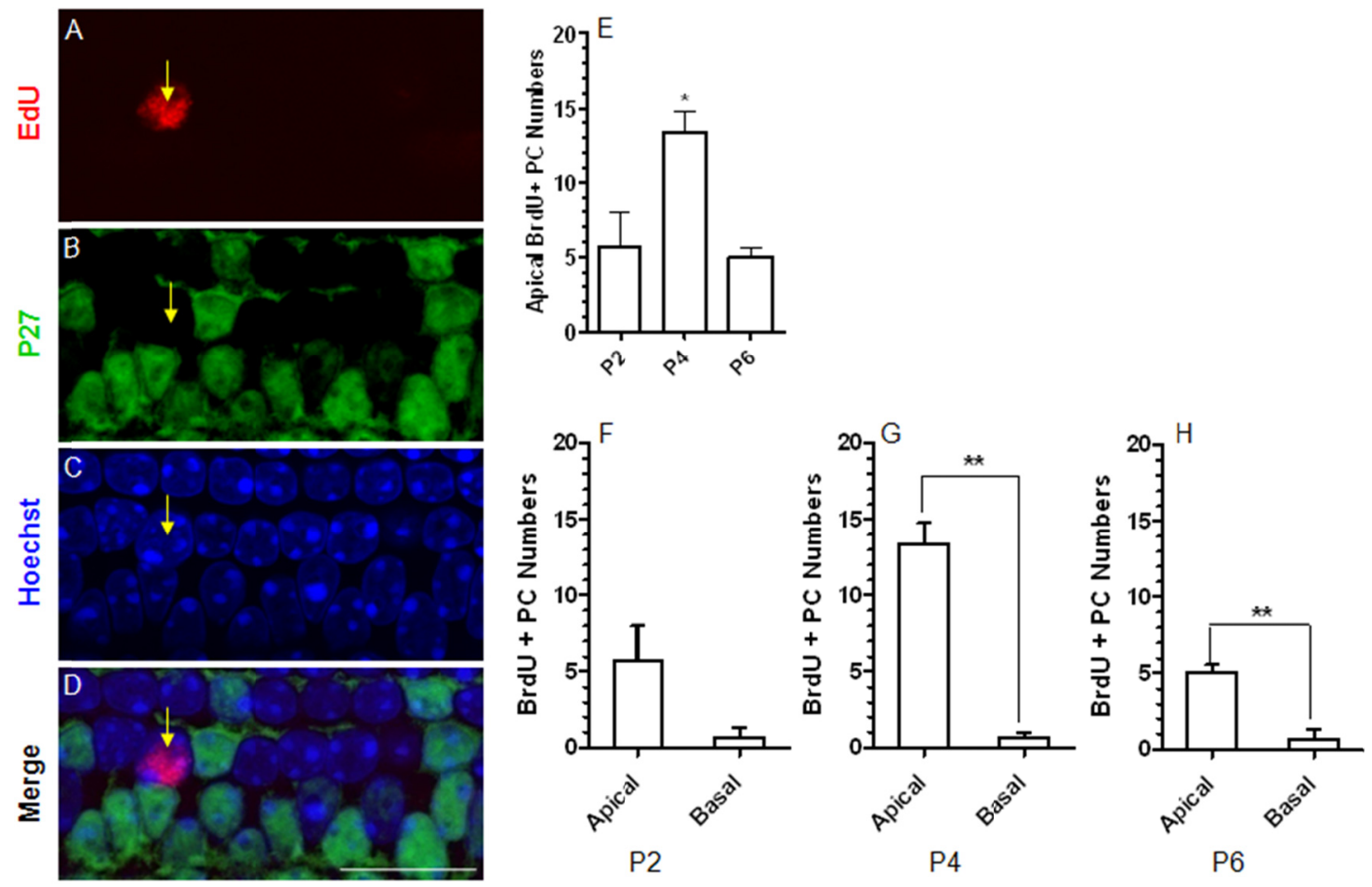

Figure 2-3 p27-null PCs proliferation peaks at $\mathrm{P4}$ in the apical turn.

Whole mount immunostaining of p27 CKO mice at P4 with EdU (A), p27 (B), Hoechst 33342 (C) and merged images (D). (E) BrdU+ PC numbers in a $200 \mu \mathrm{m}$ segment of apical turns from $\mathrm{p} 27 \mathrm{CKO}$ mice at $\mathrm{P} 2, \mathrm{P} 4$ and $\mathrm{P} 6\left(\mathrm{n}=3\right.$ for each time point, ${ }^{*} \mathrm{p}<0.05$ relative to P2 and P6). Comparison of BrdU+ PC numbers in a $200 \mu \mathrm{m}$ segment between apical and basal turns from p27 CKO mice at P2 (F), P4 (G) and P6 $(\mathrm{H}) .(n=3$ for each time point, $\left.{ }^{* *} \mathrm{p}<0.01\right)$. Scale bars: $20 \mu \mathrm{m}$. Data is expressed as mean \pm S.E.M. 
cochlea occurs in a basal to apical gradient, cells in the basal turn are a few days older than those in the apical turn. ${ }^{66}$ We took advantage of this characteristic to test the possibility that DCs may reenter S phase after P6 by analyzing the basal turn of the cochlea. Again, we did not find any BrdU+ DCs in any of the basal turns studied (data not shown). This result supports the idea that p27-null DCs may not be able to enter S phase in the neonatal mouse cochlea.

In addition, we quantified the number of BrdU+ PCs found in the basal turn from P2 to P6. There were significantly more BrdU+ PCs in the apical turn at P4 and P6 compared to basal turns and no statistical difference at P2 (Figure 2-3F-H). The difference between apical and basal turns again supports the idea that Prox 1 promoter activity and thus Cre expression is higher in the apical turn. Because P2 was the earliest age when p27-null PCs were observed to enter S phase, one might expect a large variation in the number of BrdU+ PCs, which is exactly what we found. This likely explains why there was no difference in BrdU+ PCs at P2 between basal and apical turns.

\subsection{2 p27-null pillar cells complete the cell cycle and generate daughter cells}

To determine whether $\mathrm{p} 27$-null PCs can progress through the mitosis (M) phase of the cell cycle, we used immunostaining for Phospho-Histone H3 (PH3), a marker specific for $\mathrm{M}$ phase. While there were no PH3+ cells in the control mice, PH3+ PCs were present displaying characteristics of prophase, metaphase and anaphase in the organ of Corti of p27 CKO mice at P4 (Figure 2-4). When auditory epithelia were analyzed from HC layer to SC layer by confocal microscopy, we consistently observed segregated chromosomes in a single slice. This suggests that the plane of mitosis was parallel to the luminal surface of the auditory epithelium.

The intriguing $\mathrm{PH} 3+\mathrm{PCs}$ in the $\mathrm{p} 27 \mathrm{CKO}$ mice promoted us to further determine whether p27-null PCs can further successfully undergo cytokinesis, enter G1 phase and generate new daughter cells, we examined chromosomal duplications of BrdU+ cells using the technique of Fluorescent In Situ Hybridization (FISH) with a genomic marker (a bacterial artificial chromosome clone containing Gapdh) ${ }^{80}$ Generally, cells in late S phase, G2 and M phase of the cell cycle are tetraploid and should have 4 copies of Gapdh, whereas cells in G1 and early S phases are diploid and should only have 2 copies of Gapdh; each of which state can be distinguished using FISH. ${ }^{80}$ At P4, 10 hours after the first BrdU injection, we observed 2 of 10 BrdU+ PCs with two FISH dots per nucleus, indicating that they had left $\mathrm{M}$ phase and reentered G1 phase (Figure 2-4E-H). The remaining other $8 \mathrm{BrdU}+\mathrm{PCs}$ had four FISH dots per nucleus, indicating that they were either still in late $\mathrm{S}$ phase, in G2 phase or in M phase (Figure 2-4I-L). The 2 BrdU+ cells with two FISH dots were likely to be cells that were labeled by the first BrdU injection. This confirms that p27-null PCs can complete the cell cycle and give rise to daughter cells. 

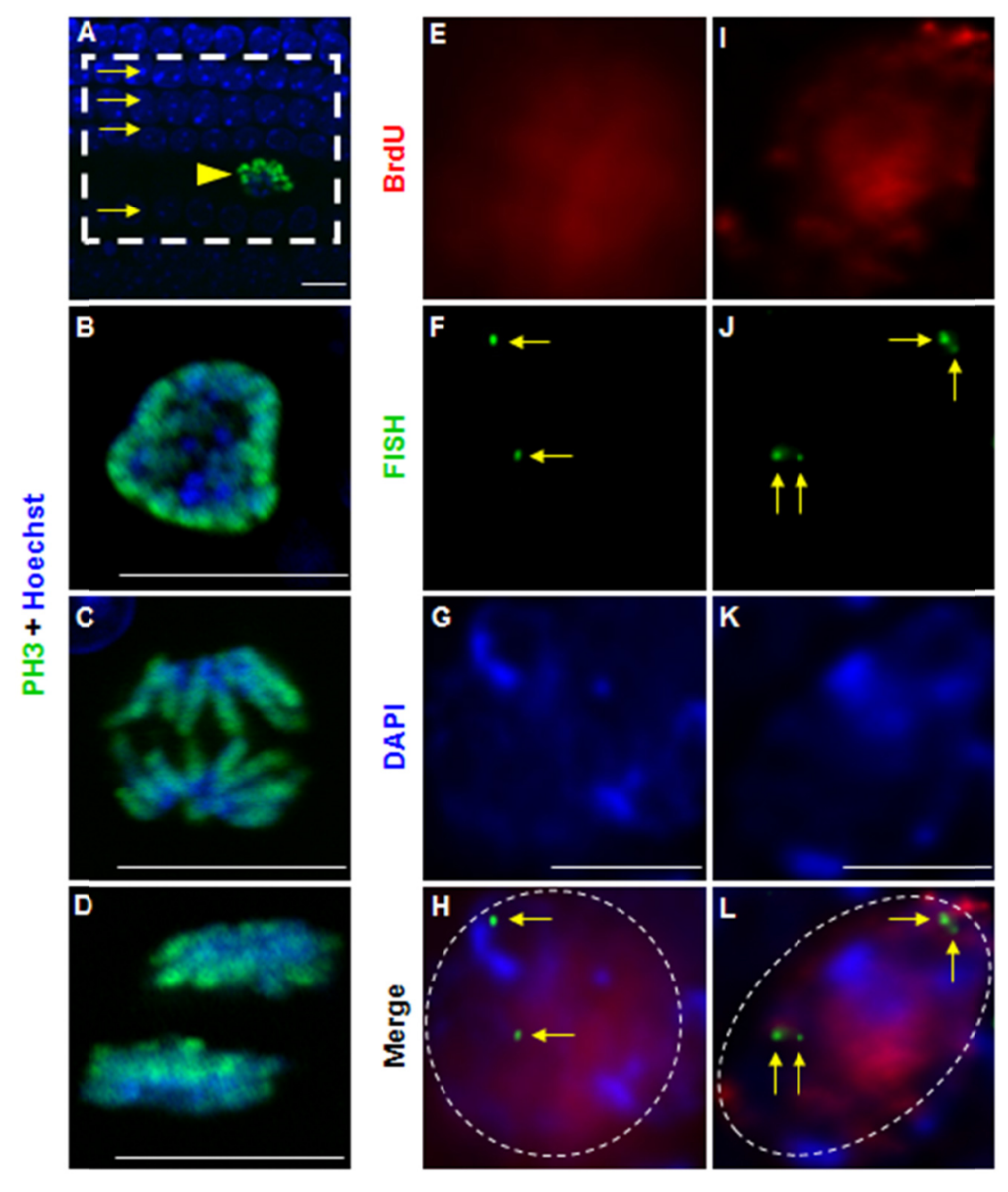

Figure 2-4 p27-null PCs progress through the M phase and generate daughter cells.

PH3 immunofluorescence (green) of PCs in the organ of Corti (A, rectangular area) in different $\mathrm{M}$ phases (B, prophase; $\mathrm{C}$, metaphase, $\mathrm{D}$, anaphase) from $\mathrm{p} 27 \mathrm{CKO}$ mice at $\mathrm{P} 4$. Hoeschst 33342 (blue) stains nuclei. Arrows in A identify three rows of outer HCs and one row of inner HCs. Arrowhead in A labels a cell that is viewed at higher magnification in B. (E-H) A BrdU+ (red) PC nucleus (DAPI in blue) with unduplicated chromosomes labeled with the FISH Gapdh probe (green dots, arrows). (I-L) A BrdU+ (red) PC nucleus (DAPI in blue) with duplicated chromosomes labeled with the FISH Gapdh probe (green dots, arrows). Dashed-line circle in H and L marked the approximate nuclear envelope. Scale bars: $10 \mu \mathrm{m}$. 


\subsection{3 p27-null daughter cells can further proliferate but continue to express supporting cell markers}

To determine whether newly born daughter cells can enter S phase, we labeled proliferating cells with BrdU (5 injections every 2 hours at P2) followed by a single EdU injection 48 hours later (at P4). BrdU and EdU assays do not cross-react. ${ }^{73}$ We found BrdU and EdU double-positive PCs, demonstrating that daughter cells were able to reenter S phase (Figure 2-5A-D). Among three mice analyzed at P4, approximately 30-40 $\mathrm{BrdU} / \mathrm{EdU}$ double-positive PCs were found in each cochlear duct. In addition, we consistently found clusters of BrdU+ cells which suggest that daughter cells could continue to proliferate and perhaps were migrating (Figure 2-5B). We also examined the total number of apical PCs and DCs relative to the total number of apical HCs at P2, P4 and P6 and found that the number of PCs continuously increased with age; whereas, the number of DCs did not change (Figure 2-5E-F). This further confirms that only p27-null PCs reentered the cell cycle and successfully completed multiple cell divisions, which is consistent with proliferating SCs in non-mammalian vertebrate $\mathrm{HC}$ regeneration. ${ }^{4}$

We consistently observed that newly formed daughter cells derived from p27-null PCs migrated upwards into the HC nuclear layer or even to the surface of the auditory epithelium, where $\mathrm{p} 75^{\mathrm{NGFR}}$ staining can be observed (Figure 2-6). This is very similar to how avian SCs behave during the $\mathrm{HC}$ regeneration process. ${ }^{81,82}$ After migration, avian SCs transdifferentiate into HCs; however, in our p27 CKO model, daughter cells continued to express Sox 2 and Prox $1,{ }^{50,51,83}$ two SC markers, suggesting that SC cell fate was maintained (Figure 2-7).

Additionally, to trace p27-null PCs, we injected tamoxifen into Prox ${ }^{\mathrm{CreER} /+}$; p2 $7^{\text {loxp/loxp }} ;$ Rosa26-EYFP ${ }^{\text {loxp/+ }}$ mice at $\mathrm{P} 0$ and $\mathrm{P} 1$, and analyzed cochlear whole mounts double-stained with EYFP and myosin-VI, a HC differentiation marker that is normally turned on earlier than myosin-VIIa. No double-positive cells for myosin-VI and EYFP were observed at P12-P13 (data not shown) after which cell death occurred. As a complementary approach, we injected BrdU daily (from P2 to P12) into p27 CKO mice that received tamoxifen injections at $\mathrm{P} 0$ and $\mathrm{P} 1$. Since endogenous $\mathrm{HCs}$ are quiescent, any cell double-positive for BrdU and myosin-VI should be generated from proliferating p27-null PCs. Again, no such cells were observed at P12. In addition, no ectopic HCs were observed between P2 to P15. These three independent lines of evidence support the idea that daughter cells of p27-null PCs maintain SC fate and that no newly formed HCs were derived from proliferating p27-null PCs in our current mouse model.

\subsubsection{Endogenous hair cells undergo cell death after P15}

There was no evidence of HCs loss in the p27 CKO cochlea at P10, while p27-null PCs underwent multiple divisions; however, considerable HC loss was found at P15, possibly caused by accumulation of proliferating PCs in the restricted space of the organ of Corti. We found that the majority of HC death occurred in the inner most rows of OHCs, with less damage in outer OHC rows that were farther away from the Tunnel of 

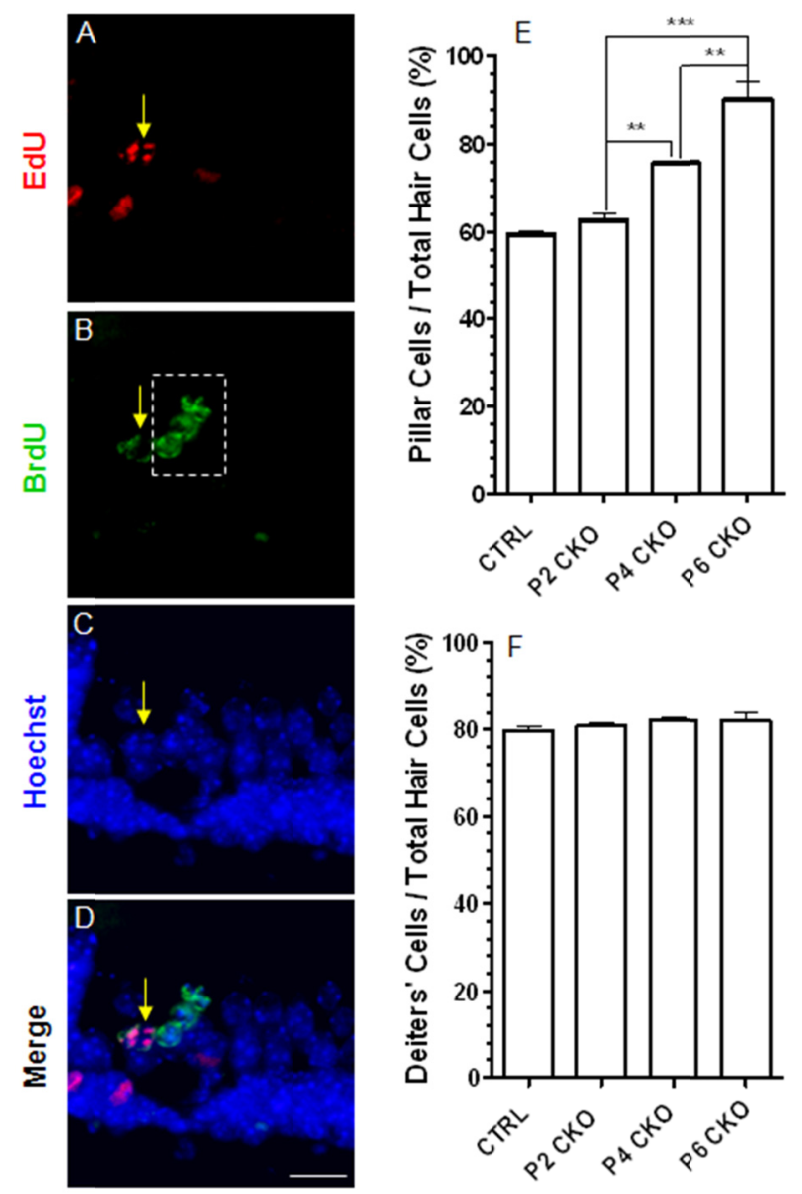

Figure 2-5 Daughter cells further proliferate.

(A-D) An EdU (red) and BrdU (green) double positive PC (arrows) from p27 CKO mice at P4. Hoeschst 33342 stains nuclei (blue). Percentage of total PC (E) and DC (F) numbers normalized to HC numbers in a $200 \mu \mathrm{m}$ length of the cochlear apical turn (p27 CKO, P2, P4 and P6; CTRL, P4; $\mathrm{n}=4-5$; ** $\mathrm{p}<0.01$; *** $\mathrm{p}<0.001)$. Scale bars: $10 \mu \mathrm{m}$. Data is expressed as mean \pm S.E.M. 


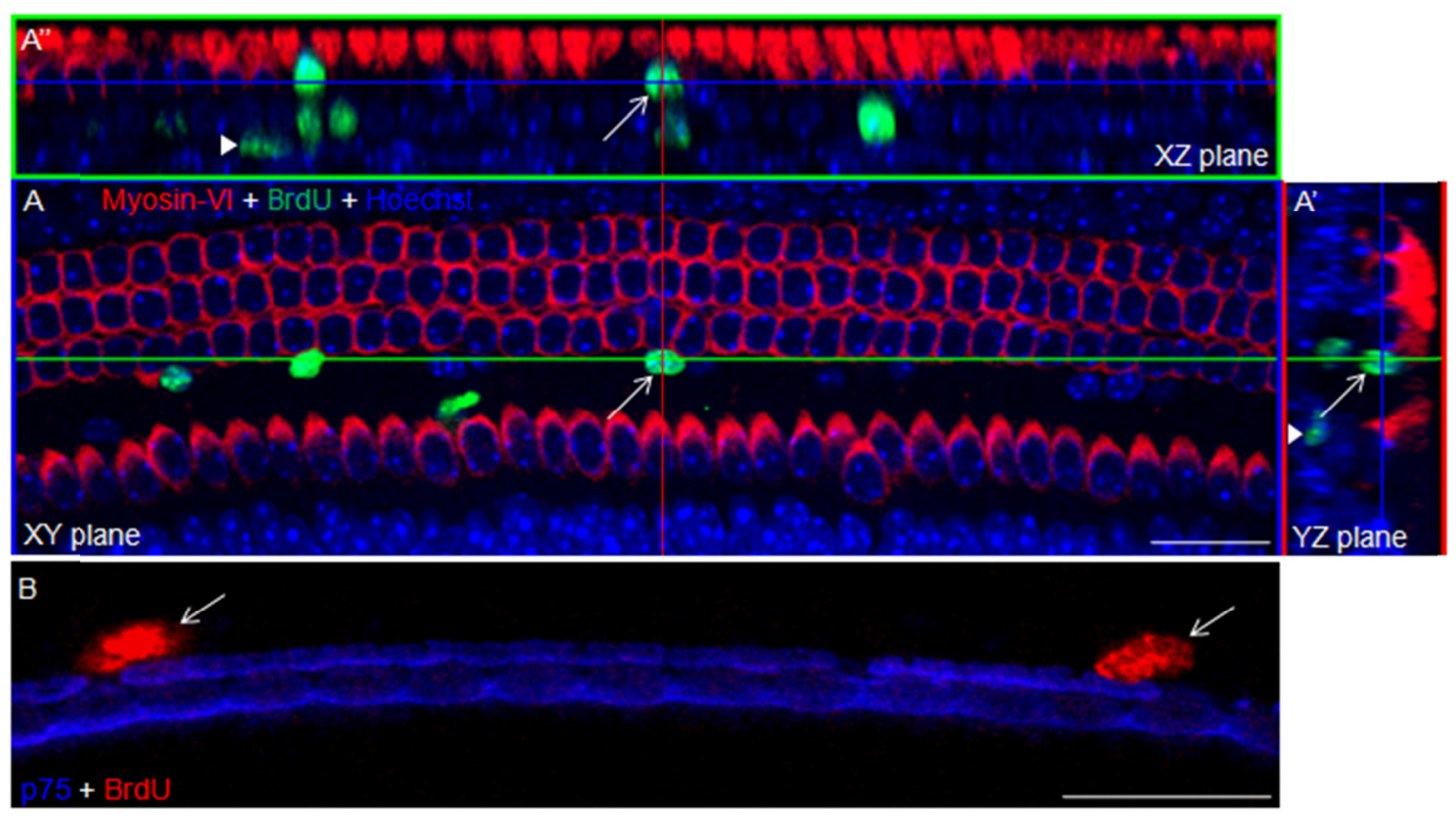

\section{Figure 2-6 Daughter cells migrate upward into the HC layer or the surface of auditory epithelium.}

Several BrdU+ (green) daughter cells were located near the Myosin VI+ (red) HC nuclear layer (blue, Hoechst33342) in p27 CKO mice at P4. White arrow identifies one daughter cell in the XY plane (A), YZ plane (A') and the XZ plane (A"). White arrow heads (A' and $A$ '): the BrdU+ cells underneath the basilar membrane. (B) The nuclei of two BrdU+ (red) daughter cells (white arrows) are located at the surface of the auditory epithelium labeled with p75NGFR (blue). Scale bars: $20 \mu \mathrm{m}$. 

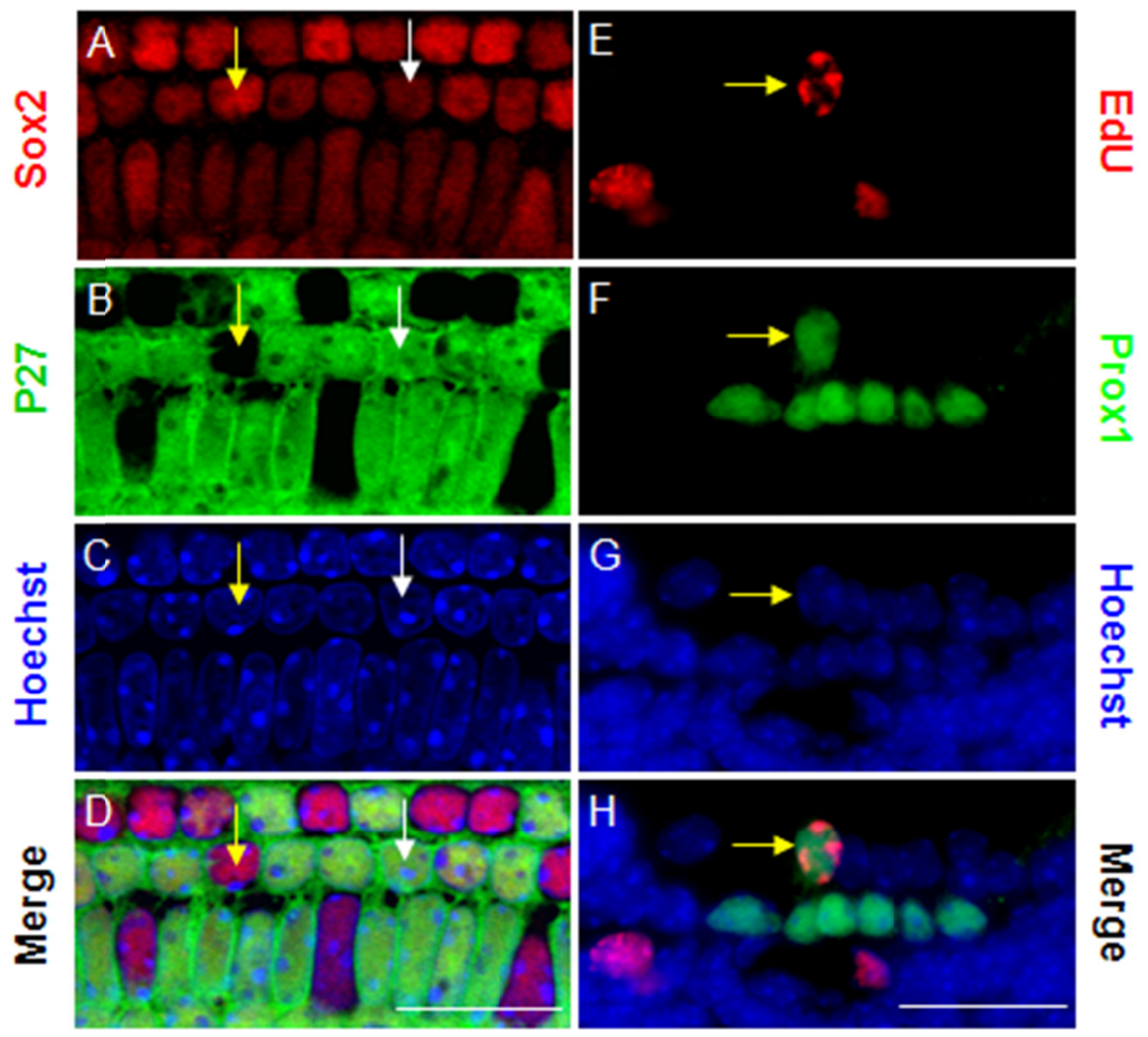

Figure 2-7 Proliferating PCs still express SC markers.

Whole mount immunostaining of the apical turn of p27 CKO mice at P2 with Sox2 (A), p27 (B), Hoechst 33342 (C) and merged images (D). Yellow arrows mark a p27-null, Sox $2+$ PC. White arrows identify a p27+, Sox $2+$ PC. Cross sections of the apical organ of Corti at P2 with immunofluorescence of EdU (E), Prox1 (F), Hoechst 33342 (G) and merged images $(\mathrm{H})$. Arrows label an EdU+, Prox 1+ PC. Similar results were observed at P4. Scale bars: $20 \mu \mathrm{m}$. 
Corti (Figure 2-8). In addition, IHCs remained largely intact at P15 and even at 6 weeks of age (data not shown). This pattern of $\mathrm{HC}$ death is correlated with accumulation of daughter cells generated from p27-null PCs in the Tunnel of Corti area. Additionally, the pattern of HC death correlates to the distance between PCs and HCs; the shorter the distance, the more $\mathrm{HC}$ death occurred. We, therefore, propose that $\mathrm{HC}$ death was caused directly or indirectly by PC proliferation. The intact state of IHCs suggests that PCs prefer to proliferate and migrate laterally towards $\mathrm{OHCs}$, but not medially towards IHCs. This is further supported by the cluster of BrdU+ PCs detected at P4 which appear to be migrating towards outer HCs.

In addition, at $\mathrm{P} 15$ but not before, we found TUNEL positive (+), myosin-VIIa-negative cells in the apical surface of the organ of Corti. These TUNEL+ cells are either dying HCs that had lost myosin-VIIa expression and been ejected from the normal HC layer or perhaps PCs that migrated upward into the HC layer. The reason why cell death occurred two weeks after the initial proliferation is not clear, although similar phenomena have been reported previously in the retina. ${ }^{84,85}$ Consistent with $\mathrm{HC}$ death observed after P10, our p 27 CKO mice exhibited a significant increased auditory brainstem response threshold at 6 weeks of age.

\subsection{Summary and Discussion}

Using the Proxl ${ }^{\mathrm{CreER} /+}$ mouse line, we have achieved in vivo proliferation of postnatal PCs by acutely deleting the cyclin-dependent kinase inhibitory protein, p27. Daughter cells of p27-null PCs undergo several rounds of division and migrate upward into the $\mathrm{HC}$ layer; however, they maintain $\mathrm{SC}$ fate and do not differentiate into HCs. The question of how to convert SCs to HCs is the topic of Chapter 4. Our work has accomplished both proliferation and migration of postnatal, mammalian SCs in vivo, two steps of the $\mathrm{HC}$ regeneration process that occurs in non-mammalian vertebrates. In addition, our model highlights SC heterogeneity as p27 is also deleted in postnatal DCs, yet we did not detect cell cycle reentry of DCs in our model.

\subsubsection{The p27 and cell cycle control in cochlear supporting cells}

The role of p27 during embryonic cochlear development is well established. It is first turned on in progenitors in the apical turn (E12.5) and last in the basal turns (E14.5) and drives the cell cycle exit of progenitors occurring in an apical-basal gradient. ${ }^{62}$ In $p 27^{-/}$germ line knockout mice, the ectopic proliferation (or delayed cell cycle exit) of progenitor cells occurs and leads to supernumerary HCs that eventually die with aging partially because of overall defective cochlear development. ${ }^{64,65}$ In postnatal cochleae of $p 27^{-/}$germ line knockout mice, supernumerary HCs with expression of $\mathrm{HC}$ specific markers are quiescent and its postmitotic state is likely to be initiated and controlled by $\mathrm{p} 19^{\text {Ink4d }}$ (also called Cdkn2d), p21 ${ }^{\text {Cip1 }}$ (also referred as to Cdkn1a), and $\mathrm{Rb} .{ }^{27,86-89}$

Hereafter we will use p19 and p21 throughout the dissertation to define $\mathrm{p} 19^{\mathrm{Ink} 4 \mathrm{~d}}$ and $\mathrm{p} 21^{\mathrm{Cip} 1}$. 


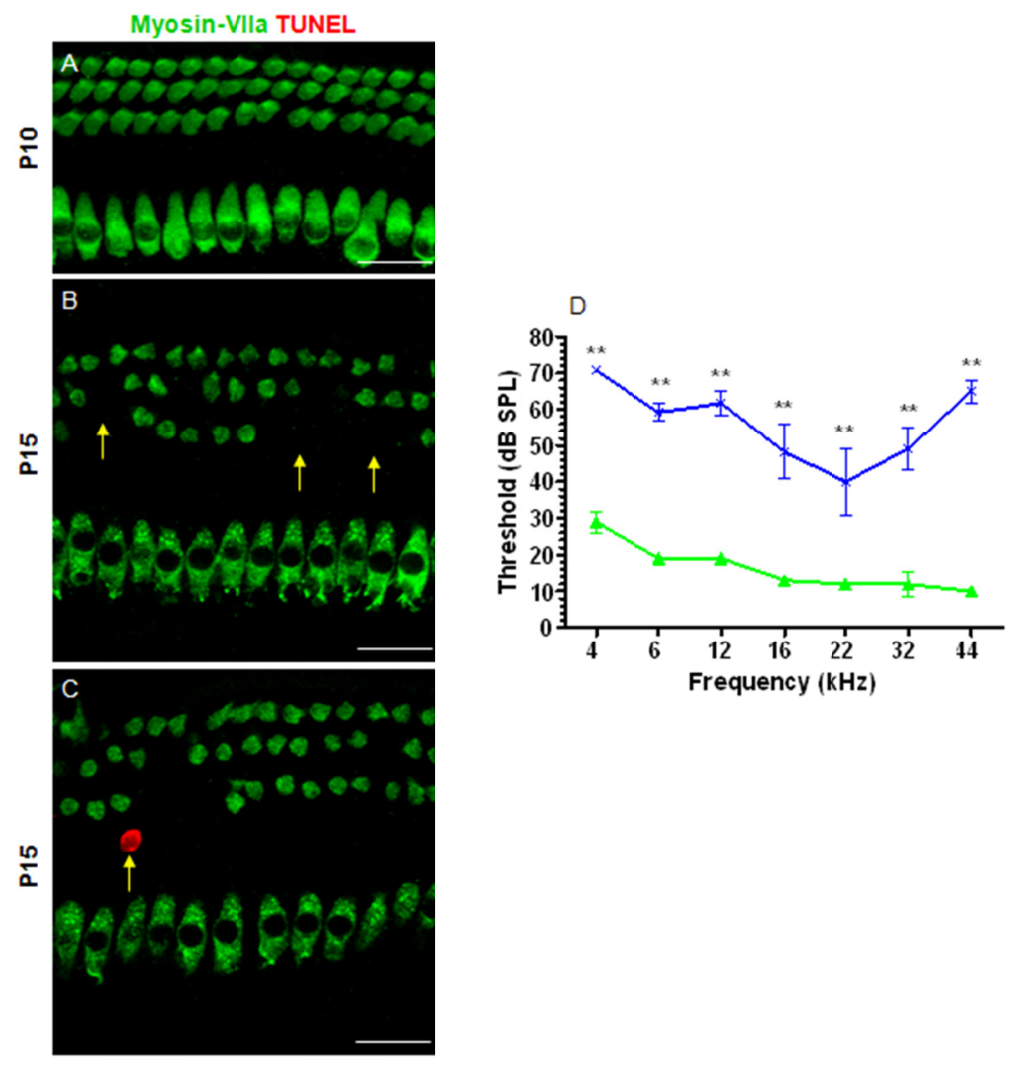

Figure 2-8 HC death and hearing loss in p27 CKO mice.

Myosin-VIIa (green) and TUNEL (red) immunofluorescence of apical cochlear whole mounts at P10 (A) and P15 (B, C) in p27 CKO mice. Arrows in B mark missing outer HCs. Arrow in C labels a dying cell that is negative for myosin VIIa staining. (D) Auditory brainstem response thresholds (dB SPL) at various frequencies $(\mathrm{kHz})$ of CTLR (green, $\mathrm{n}=5$ ) and $\mathrm{p} 27 \mathrm{CKO}$ (blue, $\mathrm{n}=6$ ) tested at 6 weeks of age $(* * \mathrm{p}<0.01)$. Scale bars: $20 \mu \mathrm{m}$. Data is expressed as mean \pm S.E.M. 
However, cells surrounding supernumerary HCs are still proliferative at postnatal ages in these mice. Because markers are not available to distinguish SC progenitors and differentiating SCs, there are two possible scenarios: the first is that the proliferative cells are SC progenitors (even in postnatal ages) which never exit the cell cycle and keep proliferation without $\mathrm{p} 27$; the second is that the proliferative cells are differentiating SCs. To distinguish between these two scenarios and clarify the roles of p27 at different postnatal ages (then cells at diverse differentiated state), it was best to delete p27 at different time points and bypass all embryonic defective development before p 27 ablation.

Recently, in vitro transfection of neonatal cochleae with vectors expressing p27 shRNA shows the importance of $\mathrm{p} 27$ in the SCs distributed in the LER region. ${ }^{90}$ However, SCs (PCs and DCs) inside the organ of Corti which are cell types we focused on in current study are difficult to transfect. Therefore, we took the advantages of the conditional genetic mouse models in which we not only could target PCs and DCs but also at different ages. When $p 27$ was specifically deleted at $\mathrm{P} 0 / \mathrm{P} 1$, to our surprise, only p27-null PCs could proliferate which was different from presence of proliferative PCs and DCs in $p 27^{-/}$germ line knockout mice. ${ }^{65}$ It might support that the SCs in $p 27^{-/}$germ line knockout mice are more likely to be progenitor cell state and highlight the intrinsic, different differentiated states between neonatal SCs without $\mathrm{p} 27$ in our $p 27$ conditional model and those without $\mathrm{p} 27$ from the beginning of their birth in $p 27^{-/}$germ line knockout mice. $^{65}$

\subsubsection{Comparison with avian $\mathrm{HC}$ regeneration}

After HC damage in the avian basilar papilla, SCs proliferate and migrate upwards into the HC layer. ${ }^{4}$ Daughter cells that differentiate into HCs remain in the HC layer, whereas daughter cells that maintain SC fate migrate again, downward to the SC layer. ${ }^{81}$ In our current model, p27-null PCs behave similarly by proliferating and migrating into the $\mathrm{HC}$ nuclear layer or even to the surface of the auditory epithelium. The difference, however, is that these daughter cells still express SC markers (Prox1 and Sox2), thus do not differentiate into HCs.

It is possible that endogenous HCs prevent $p 27-n u l l ~ P C s$ from differentiating into $\mathrm{HCs}$ in a similar fashion to what occurs during embryonic development, where developing $\mathrm{HCs}$ prevent neighboring cells from also committing to $\mathrm{HC}$ fate by lateral inhibition. ${ }^{14,24}$ Similarly, in the avian basilar papilla, $\mathrm{HC}$ regeneration does not occur unless $\mathrm{HC}$ damage happens first. ${ }^{4}$ Unfortunately, there are no effective means of damaging $\mathrm{HCs}$ in neonatal mice in vivo. Drugs-induced $\mathrm{HC}$ damage, using aminoglycoside antibiotics or platinum-based chemotherapy agents, are effective in adult mice or guinea pigs, ${ }^{51}$ but in neonates, result in death of the animal before $\mathrm{HC}$ death (our unpublished observation). Noise damage is also not an option as hearing is not fully mature until $\sim \mathrm{P} 21 .^{63}$

Another possible explanation for the lack of newly formed HCs in our model is 
that $p 27$-null daughter cells still express Sox 2 and Prox1. It has recently been shown that both of these proteins suppress the activity of Atoh $1,{ }^{48}$ a transcription factor that is both necessary and sufficient for $\mathrm{HC}$ differentiation. ${ }^{38}$ Additionally, a recent report indicates that the cell fate of neonatal PCs is maintained by two pathways, the Notch pathway and the FGF pathway, during which hey2 is shown to be a key effector. ${ }^{91}$ Given such strong control of PC fate, it is likely that the expression of other differentiation proteins (i.e. Atoh1 to be discussed in Chapter 5) needs to be manipulated in order for $p 27$-null PCs to differentiate into HCs. 


\section{CHAPTER 3. SOX2 IS REQUIRED TO MAINTAIN QUIESCENCE OF INNER PILLAR CELLS}

\subsection{Introduction}

In Chapter 2, we showed that acute ablation of cell cycle inhibitor p27 leads to proliferation of PCs. However, these $p 27$-null PCs maintain the postnatal SC marker Sox 2 and Prox1, suggesting that the SC cell fate is maintained. Because Sox 2 is suggested to inhibit activities of Atoh1 during the embryonic cochlear development, ${ }^{48}$ we propose that $\mathrm{Sox} 2$ is required in maintaining the $\mathrm{SC}$ fate at postnatal ages and that the deletion of Sox 2 will lead to the conversion of SCs into HCs.

Sox2 germ line knockout mice are lethal at very early embryonic ages. ${ }^{92}$ In addition, two Sox 2 hypomorphic mouse lines, $S o x 2^{Y s b / Y s b}$ and $S o x 2^{L c c / L c c}$, have severely defective cochlear development ${ }^{46}$. To bypass this limitation, we analyzed the Sox2 loxp/loxp mouse line that has been described previously. ${ }^{93}$ To specifically delete Sox 2 at different postnatal ages, we characterized the tamoxifen inducible iCre (improved Cre) activity of Fgfr $3^{\text {iCreER+ }}$ mice in cochlear cells. ${ }^{74}$ The majority of the iCre activity is limited to PCs and DCs when tamoxifen is given at postnatal ages.

Contrary to our prediction, we did not observe a cell fate switch of Sox2-null PCs (can be subdivided into inner pillar cells and outer pillar cells) or DCs. In contrast, we found that Sox2-null inner pillar cells (IPCs) down-regulated p27, proliferated, while outer pillar cells (OPCs) and DCs were still quiescent and maintained normal expression of $\mathrm{p} 27$.

\subsection{Materials and Methods}

\subsubsection{Mouse strains}

Sox $2^{\text {loxplloxp }}$ mouse line was generated in Dr. Okuda Akihiko laboratory in Saitama Medical University (Japan). Fgfr $3^{\text {CreER }}{ }^{+}$mouse line was generated in Dr. William Richardson laboratory at the University College London (UK). CAG-EGFP+ mice were kindly provided by Dr. Jeffrey Robbins from the Cincinnati Children's Hospital Medical Center, Hes $5^{\text {LacZ/+ }}$ mice were kindly provided by Dr. Ryoichiro Kageyama from Kyoto University. Rosa26-CAG-Tdtomato ${ }^{\text {loxp } /+}$, Notch $1^{\text {loxplloxp }}$ and Rosa26-EYFP $P^{l o x p / t}$ mice were purchased from Jackson laboratory (USA).

To characterize iCre activity, $\mathrm{Fg} f \mathrm{r} 3^{i \mathrm{CreER+}} ;$, Rosa26-EYFP $\mathrm{P}^{\text {loxp } /+}$ mice were treated with tamoxifen at $\mathrm{P} 0$ and $\mathrm{P} 1$, and analyzed at $\mathrm{P} 6 ; \mathrm{Fg} f \mathrm{r}^{i \mathrm{CreER}^{+}} ; \mathrm{CAG-EGFP+ \text {mice }}$ were treated with tamoxifen at $\mathrm{P} 6$ and $\mathrm{P} 7$, and analyzed at $\mathrm{P} 12 ; \mathrm{Fg} f \mathrm{r}^{\text {iCreER+ }}{ }^{\text {; }}$; Rosa26-CAG-Tdtomato ${ }^{\text {loxp } / 4}$ mice that were treated with tamoxifen at $\mathrm{P} 30$ and analyzed at P40. Sox $2^{\text {loxp/loxp }}$ mice were bred with Fgfr $3^{3^{\text {CreER+ }}}$ mice to get Fgfr $3^{i \text { CreER+ }}$; Sox $2^{\text {loxp } /+}$. 
Fgfr $3^{\text {iCreER+ }}$; Sox $2^{\text {loxp } /+}$ mice were interbred to get $F g f r 3^{i C r e E R+}$; Sox $2^{\text {loxp/loxp }}$ mice as the experimental group, while $\mathrm{Fg} f \mathrm{r} 3^{i \mathrm{CreER+}}$; Sox $2^{+++}$mice in the same littermate were used as the control group.

\subsubsection{Tissue preparation, immunofluorescence and analysis}

Detailed protocols have been described in Chapter 2. The following primary antibodies were used: anti-Myosin-VI (rabbit, 1:200, 25-6791, Proteus Bioscience, Ramona, CA), anti-Myosin-VIIa (rabbit, 1:200, 25-6790, Proteus Bioscience), anti-Prox1 (rabbit, 1:500, AB5475, Millipore, Billerica, MA), anti-Calbindin ( rabbit,1:500, AB1778, Millipore), anti-p75NGFR (rabbit, 1:1000, AB1554, Millipore), anti-GFP (chicken, 1:1000, ab13970, Abcam, Cambridge, UK), anti-p27 (mouse, 1:500, 610242, BD Transduction Laboratories, Franklin Lakes, NJ), anti-Sox2 (goat, 1:1000, sc-17320, Santa Cruz Biotechnology, Santa Cruz, CA), and Alexa Fluor 488-conjugated anti-pH3 (rabbit, 1:20, 9708, Cell Signaling, Danvers, MA). The following secondary antibodies were used: donkey anti-rabbit Alexa Fluor 647 (1:1000, A31573, Invitrogen, Carlsbad, CA), donkey anti-chicken DyLt 488 (1:200, 703-486-155, Jackson ImmunoResearch, West Grove, PA), donkey anti-goat Alexa Fluor 568 (1:1000, A11057, Invitrogen), goat anti-rabbit Alexa Fluor 647 (1:1000, A21245, Invitrogen), goat anti-rabbit Alexa Fluor 568 (1:1000, A11036, Invitrogen), and goat anti-chicken Alexa Fluor 488 (1:1000, A11039, Invitrogen).

Note that for p27 whole mount staining; we performed an antigen retrieval process (H-3300, Vector Laboratories, Burlingame, CA), followed by the Tyramide Signal Amplification Kit (T20912, Invitrogen) protocol recommended by Invitrogen. For cell death measurements, TUNEL staining was performed according to the protocol provided with the In Situ Cell Death Detection Kit, Fluorescein/TMR Red (11684795910/12156792910, Roche Applied Science, Indianapolis, IN).

\subsubsection{Luciferase assays and cell lines}

Plasmids for the $p 27$ promoter driving the luciferase and the empty luciferase control were obtained from Dr. Toshiyuki Sakai, Kyoto Prefectural University of Medicine, Kyoto, Japan. LacZ, E2F1, and Sox2 expression vectors were obtained from Addgene (Cambridge, MA, plasmid 18816, 10736 and 13459). Luciferase and beta-galactosidase activity were assayed using the Applied Biosystems Dual-Light kit (Carlsbad, CA) and quantitated on a Promega (Madison, WI) Glomax Multi plate reader. Plasmids were co-transfected into approximately 10,000 MEF/HELA/HEK cells utilizing Lipofectamine LTX (Invitrogen, Carlsbad, CA), following the manufactures protocol at a 7:1 ratio of LTX to DNA. HEK and immortalized MEF cells were obtained from Dr. Kundu (St. Jude Children's Research Hospital). HELA cells were obtained from ATCC (Manassas, VA). 


\subsubsection{Chromatin immunoprecipitation (ChIP) assay}

ChIP was performed utilizing the Simple ChIP Magnetic kit (9003, Cell Signaling, Boston, MA). DNA was immunoprecipitated utilizing the ChIP formulated Sox2 antibody (5024, Cell Signaling, Boston, MA), or the H3 antibody (5275, positive control), liberated, purified and probed utilizing primers specific for either the putative Sox2 binding site (Forward: 5'-CAAGGCCTGCTTAGGATACT-3', Reverse: 5'-GGGGTTTTATTTATGACCTC-3') or RPL30 (Cell Signaling).

\subsubsection{Auditory brainstem response and statistical analyses}

Detailed information was described in Chapter 2.

\subsection{Results}

\subsubsection{Neonatal inner pillar cells proliferate after acute deletion of Sox2}

In the neonatal and adult mouse cochlea, Sox2 is highly expressed in SCs inside the organ of Corti, in cells of the greater epithelium ridge (GER), and in Hensen cells lateral to the organ of Corti. Inside the organ of Corti, there are two types of pillar cells (PCs), inner pillar cells (IPCs) and outer pillar cells (OPCs), and Deiters' cells (DCs), all of which reside underneath the auditory HCs (Figure 3-1A). To determine the roles of Sox 2 in IPCs, OPCs and DCs, we first characterized the $\mathrm{Fg} F \mathrm{r}^{3{ }^{i \mathrm{CreER}+}}$ transgenic mouse line by crossing it with the Rosa26-EYFP ${ }^{l o x p /+}$ reporter mouse line. Fgfr $3^{i \mathrm{CreER+}}$; Rosa26-EYFP ${ }^{\text {loxp } /+}$ mice were given tamoxifen at $\mathrm{P} 0$ and $\mathrm{P} 1$ and analyzed at $\mathrm{P} 6$. There were many EYFP+ cells throughout the entire cochlea (Figure 3-1B-D'). Inside the organ of Corti, the majority of EYFP+ cells were Prox 1+ IPCs, OPCs and DCs, and a very small fraction were OHCs, Hensen and Claudius cells (Figure 3-1E). Note that EYFP+ IHCs were never found. When EYFP+ DCs, PCs or OHCs were normalized to the total number of DCs, PCs or OHCs in the same confocal scanning region $(n=3)$, approximately $70 \%$ of PCs and DCs were EYFP+, and only $1 \%-2 \%$ of OHCs were EYFP+ (Figure $3-1 \mathrm{~F})$. In addition, in each $\sim 160 \mu \mathrm{m}$ cochlear duct, a few $(2 \pm 2, n=3)$ EYFP+ Hensen or Claudius cells were frequently observed in the lesser epithelium ridge (LER), which are not the cell types of our focus here. Although iCre activity of $\mathrm{Fgfr} 3^{i \mathrm{CreER}+}$ mice is not $100 \%$ specific to SCs, it is suitable for our study because Sox 2 is undetectable in all postnatal HCs.

Next, we crossed Fgfr $3^{\text {iCreER+ }}$ with Sox $2^{\text {loxplloxp }}$ mice and gave $F g f r 3^{\text {iCreER+ }}$; Sox 2 loxp/loxp pups tamoxifen at P0 and P1, EdU at P2 and analyzed their cochleae 6 hours later. While HCs remained intact, Sox 2 was deleted in IPCs, OPCs and DCs across the entire cochlea and many EdU+ cells were identified. We quantified EdU+ cells along the entire cochlea $(5544 \mu \mathrm{m} \pm 88 \mu \mathrm{m}, n=3)$. In each sample, there were $86 \pm 11(n=3)$ EdU+ cells. Among the total $259 \mathrm{EdU}+$ cells in three samples (all of which were Sox2-negative), 

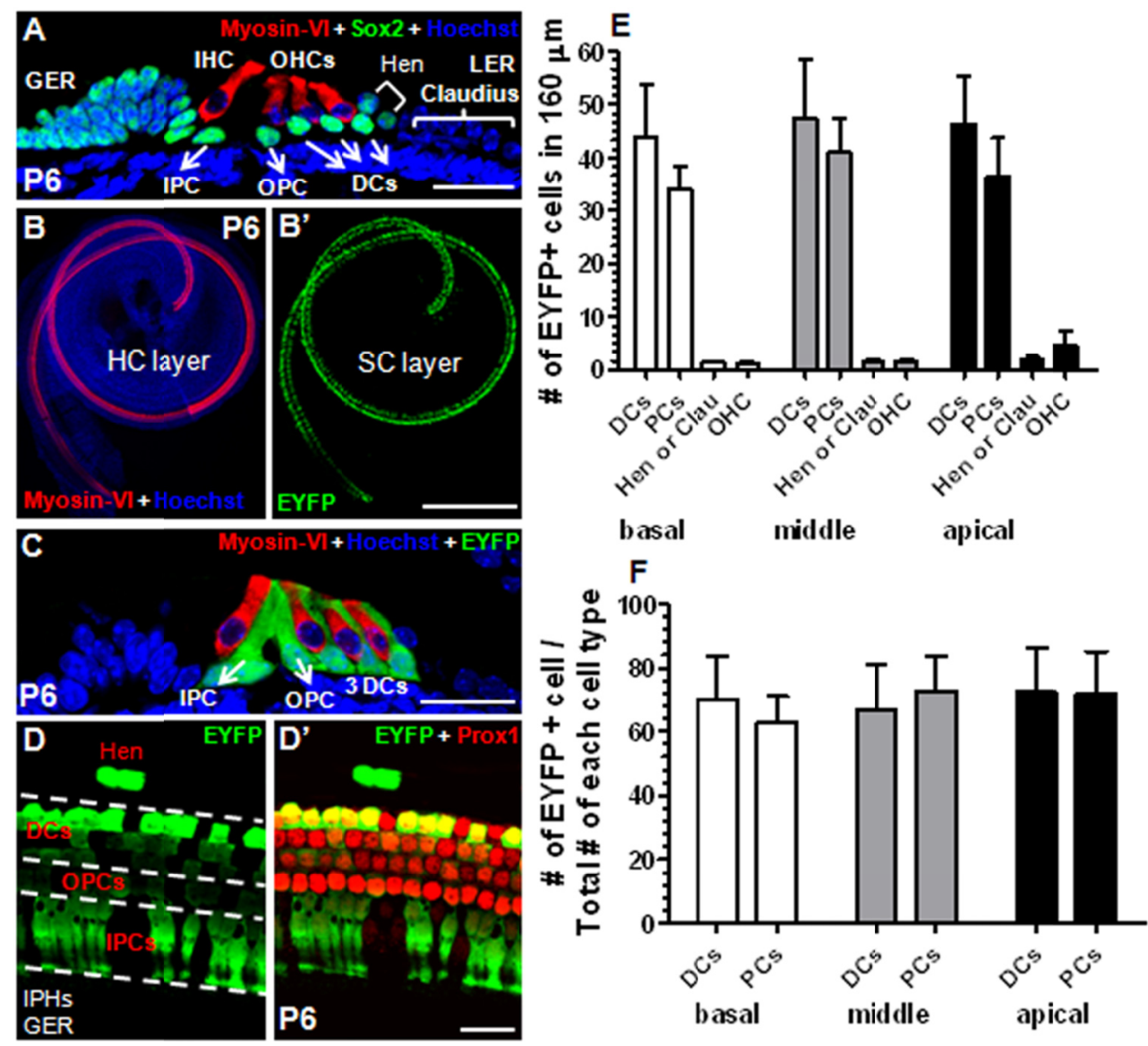

Figure 3-1 High iCre activity of Fgfr $3^{\mathrm{iCreER+}}$ mice.

(A) Histology of mouse organ of Corti. (B-C) Whole mount image at HC layer (B) and SC layer (B'), or trans-section image (C) of cochlear sample from $\mathrm{Fg} f \mathrm{r}^{\mathrm{iCreER+}}{ }^{+}$;

Rosa26-EYFP ${ }^{l o x p /+}$ mice that were subjected to tamoxifen at P0/P1 and analyzed at P6. (D-D') Double labeling of Prox 1 and EYFP at SC layer of cochlea. EYFP expression was not found in IPHs and cells in GER region. (E) Quantification of EYFP+ cells in $\sim 160$ $\mu \mathrm{m}$ cochlear length. (F) Proporation of EYFP + cells among the total number of each cell type. OHCs: outer hair cells; IHC: inner hair cell; IPC: inner pillar cell; OPC: outer pillar cell; DCs: Deiters' cells; IPHs: inner phalangeal cells; GER: greater epithelium ridge; LER: lesser epithelium ridge; Hen: Hensen cells; Bars: 20 m (A, C, D'); $200 \mu \mathrm{m}$ (B). 
$81 \%(210 / 259)$ were in the apical turn, $19 \%(49 / 259)$ in the middle turn, while no EdU+ cells were found in the basal turn. Intriguingly, all of the 259 EdU+ cells were IPCs which were defined by their unique oblong shape and location (Figure 3-2A-B'). This implies that ablation of Sox 2 at P0 and P1 leads to S phase reentry only in IPCs. In control mice, Sox 2 was expressed in all PCs and DCs, and consequently there were no EdU+ SCs observed (Figure 3-2C). As an internal positive control, EdU + mesenchymal cells underneath SCs were found in control mice (Figure 3-2C').

When $\mathrm{Fg} f \mathrm{r} 3^{\text {iCreER+ }}$; Sox $2^{\text {loxp } / \text { loxp }}$ mice were given tamoxifen at P0 and P1, EdU at P4 and analyzed 6 hours after EdU injection, we found EdU+ IPCs but no other SC subtypes across the whole turns were labeled by EdU. Strikingly, when triple staining of p27, Sox 2 and EdU was performed, we observed EdU+/Sox2-negative/ p27-negative IPCs ( $8 \pm 3$, averaged in 9 randomly picked $160 \mu \mathrm{m}$ length of cochlea in three samples) across the entire cochlea at P4 (Figure 3-2D-D"'). Faint but detectable p27 was present in a few Sox2-negative/ EdU-negative IPCs (arrow heads in Figure 3-2D-D"' '). It highlights the delay between Sox 2 ablation and complete degradation of the remaining p27 protein; however, all EdU+ IPCs were Sox2-negative/p27-negative. We did not analyze older ages because we were no longer confident in our ability to distinguish OPCs from new daughter cells produced by the proliferating Sox2-negative IPCs. Interestingly, expression of p27 was still maintained in all Sox2-negative OPCs and DCs (Figure 3-2D-D"'), which might account for why only Sox2-negative IPCs could proliferate. To further determine whether EdU+ IPCs could also enter the mitotic (M) phase, we performed double staining of EdU and phospho-histone 3 (pH3, mitotic marker) and a few EdU+/ pH3+ IPCs were observed (Figure 3-2E). This suggests that Sox2-negative IPCs are able to finish S phase and enter M phase within 6 hours. Taken together, these findings demonstrate that Sox 2 is required to maintain the quiescence of IPCs at neonatal ages.

Sox 2 is believed to be an upstream regulator of Prox 1 at embryonic ages. We determined the Prox1 expression pattern in Sox2-negative SCs, and found that Prox1 was maintained in all Sox2-negative IPCs, OPCs and DCs. This suggests that Sox2 is dispensable in maintaining Prox 1 expression by P4 (Figure 3-2F-G'). We did not analyze samples at older ages due to the fact that Prox1 expression is down-regulated with age under control conditions. Finally, in all cochlear samples analyzed at both P2 and P4, we never observed EdU+/Myosin-VI+ cells or extra HCs. It not only supports our assumption that deletion of Sox 2 in $1 \%-2 \%$ of Cre+ $\mathrm{OHCs}$ does not result in a detectable phenotype, but also suggests that no new HCs were generated upon Sox2 deletion in SCs.

\subsubsection{Juvenile inner pillar cells proliferate after acute deletion of Sox2}

Because cochlear development advances significantly during the first week after birth, we deleted Sox2 in juvenile SCs (P6 and P7) and tested whether they still need Sox 2 to remain quiescent. To measure the amount of iCre activity upon tamoxifen induction at $\mathrm{P} 6$ and P7, we crossed $C A G-E G F P+$ with $F g f r 3^{i C r e E R+}$ mice. $F g f r 3^{i C r e E R+}$; 


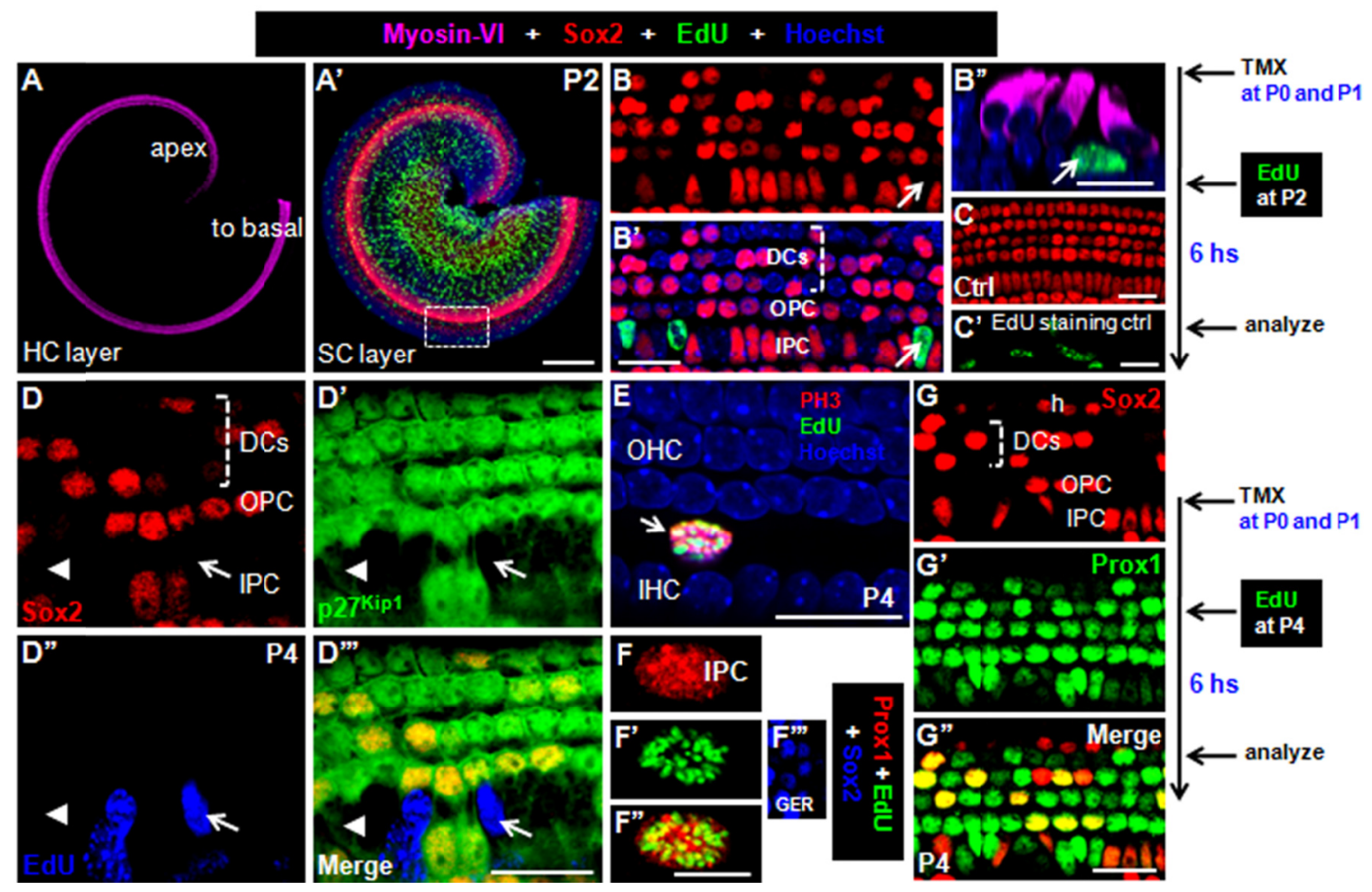

Figure 3-2 Deletion of Sox2 leads to proliferation of neonatal inner pillar cells (IPCs).

(A-B') Triple staining of Myosin-VI, Sox2 and EdU at HC layer (A) and SC layer (A'). (B-B') is the high-magnified image of the white rectangular area in (A'). Arrows point to the same EdU+/ Sox2- negative IPC. (C-C') Normal expression of Sox2 at SC layer (C) and presence of EdU+ cells in the mesenchymal cell layer $\left(C^{\prime}\right)$ in the same control sample. (D-D"' ') Triple staining of Sox2, p27 and EdU. Arrows point to the same EdU+ / Sox2-negative / p27-negative IPC. Arrow heads point to the same Sox2-negative IPC with faint p27 expression which is EdU- negative. (E) The PH3+/ EdU+ IPC (arrow) migrates to HC layer. (F-F',') Triple staining of Prox1, EdU and Sox2. The EdU+ I Sox2-negative IPC maintains Prox1 expression. ( $F^{\prime \prime}$ ') is the image of GER cells of the same sample, used as positive control for Sox2 staining. (G-G'') Image of double staining of Sox 2 and Prox1. Prox1 is maintained in all Sox 2 -negative SCs. OHCs: outer hair cells; IHCs: inner hair cells; IPC: inner pillar cell; OPC: outer pillar cell; DCs: Deiters' cells; h: Hensen cells. Bars: $200 \mu \mathrm{m}$ (A'); $20 \mu \mathrm{m}$ (B'-D',', E, G' '); $10 \mu \mathrm{m}$ (F', '). 
$C A G-E G F P+$ mice were given tamoxifen at $\mathrm{P} 6$ and $\mathrm{P} 7$ and analyzed at $\mathrm{P} 15$. We switched to $C A G-E G F P+$ reporter mice because we found that the Rosa26 promoter activity becomes weaker with age in the cochlea. Again, the majority of EGFP+ cells in the organ of Corti were IPCs, OPCs and DCs, while a few OHCs, Henson and Claudius cells were also EGFP+. Approximately $93 \%$ of PCs and DCs were EGFP+ (Figure 3-3A-B). We then used $\mathrm{Fgfr} 3^{i \mathrm{CreER}+}$; Sox $2^{\text {loxp/loxp }}$ mice as the experimental group and $\mathrm{Fg} f \mathrm{r} 3^{\text {iCreER+}}$; Sox $2^{+/+}$mice as the control group, both of which received the same treatments.

Fgfr $3^{\text {iCreER+ }}$; Sox $2^{\text {loxp/loxp }}$ mice were treated with tamoxifen at P6 and P7, EdU at P8 and analyzed 6 hours after EdU injection at P8 (Figure 3-3C-C' '). In each cochlea, there were $45 \pm 12(n=3)$ EdU+ cells. In three cochleae $(5733 \mu \mathrm{m} \pm 75 \mu \mathrm{m})$, a total of $137 \mathrm{EdU}+$ cells were found throughout the entire cochlea and all were Sox2-negative. Of these, $34.3 \%(47 / 137)$ were distributed in the apical turn, $37.1 \%(51 / 137)$ in the middle turn and $28.5 \%(39 / 137)$ in the basal turn. All EdU+ cells were IPCs, again confirming that Sox2-negative IPCs reentered S phase after acute Sox2 deletion (Figure 3-3C' '). We were still confident in our ability to define EdU+ cells as IPCs because of their location and the unique structure of organ of Corti at P8. In addition, a few EdU+/ $\mathrm{pH} 3+/$ Sox2-neative IPCs $(8 \pm 2, n=3)$ were observed (Figure 3-3D-D','). Furthermore, triple staining of EdU, Sox 2 and p27 showed that p27 became undetectable in all EdU+/ Sox2-negative IPCs. Again, while the vast majority of Sox2-negative IPCs were also p27-negative (arrows in Figure 3-3E-E','), some Sox2-negative IPCs still had a detectable level of p27 (arrow heads in Figure 3-3E-E'"'). This observation is either due to a delay between Sox 2 ablation and elimination of $\mathrm{p} 27$, or suggests the possibility that a small minority of IPCs do not need Sox 2 to maintain p27 expression at P6 and P7. Note that both Sox2+ and Sox2-negative OPCs and DCs continued to express p27, consistent with the absence of EdU in these cell types (Figure 3-3E-E','). It is worthy to highlight that the total number of EdU+ IPCs here (137 in three cochleae) was much less than the total number of EdU+ IPCs when Sox 2 was deleted at P0 and P1 (259 in three cochleae). Because more SCs are Cre+ when tamoxifen was given at P6 and P7 (93\%) than at P0 and P1 (70\%), it suggests that proliferative capacity of IPCs likely declines with maturation. In addition, no EdU+/Calbindin (another $\mathrm{HC}$ marker) + cells or extra HCs were found, which again illustrates that deletion of Sox 2 in a few Cre+ OHCs does not cause a phenotype and that Sox2-negative SCs did not switch into HCs. Finally, in the control mice, we did not find any EdU+ or pH3+ cells inside the organ of Corti (data not shown).

\subsubsection{Sox2 deletion in adult inner pillar cells leads to limited proliferation}

Because Sox 2 expression is maintained in adult $\mathrm{SCs},{ }^{50}$ we acutely deleted Sox 2 in adult SCs at P30. To characterize Cre activity at this age, $\mathrm{Fgfr} 3^{{ }^{\mathrm{CCeER+}} \text {; }}$

Rosa26-CAG-Tdtomato ${ }^{\text {loxp } /+}$ mice were given tamoxifen once at $\mathrm{P} 30$ and analyzed at P40 (Figure 3-4A). In contrast to our tamoxifen induction at neonatal and juvenile ages, all Tdtomato+ cells were SCs, which highlights the specificity of Cre activity in the Fgfr $3^{i C r e E R+}$ mice at adult ages. Approximately $95 \%$ of all DC and PC were Tdtomato+, demonstrating the high efficiency of one tamoxifen injection. Fgfr $3^{\text {iCreER+ }}$; Sox $2^{\text {loxp/loxp }}$ 


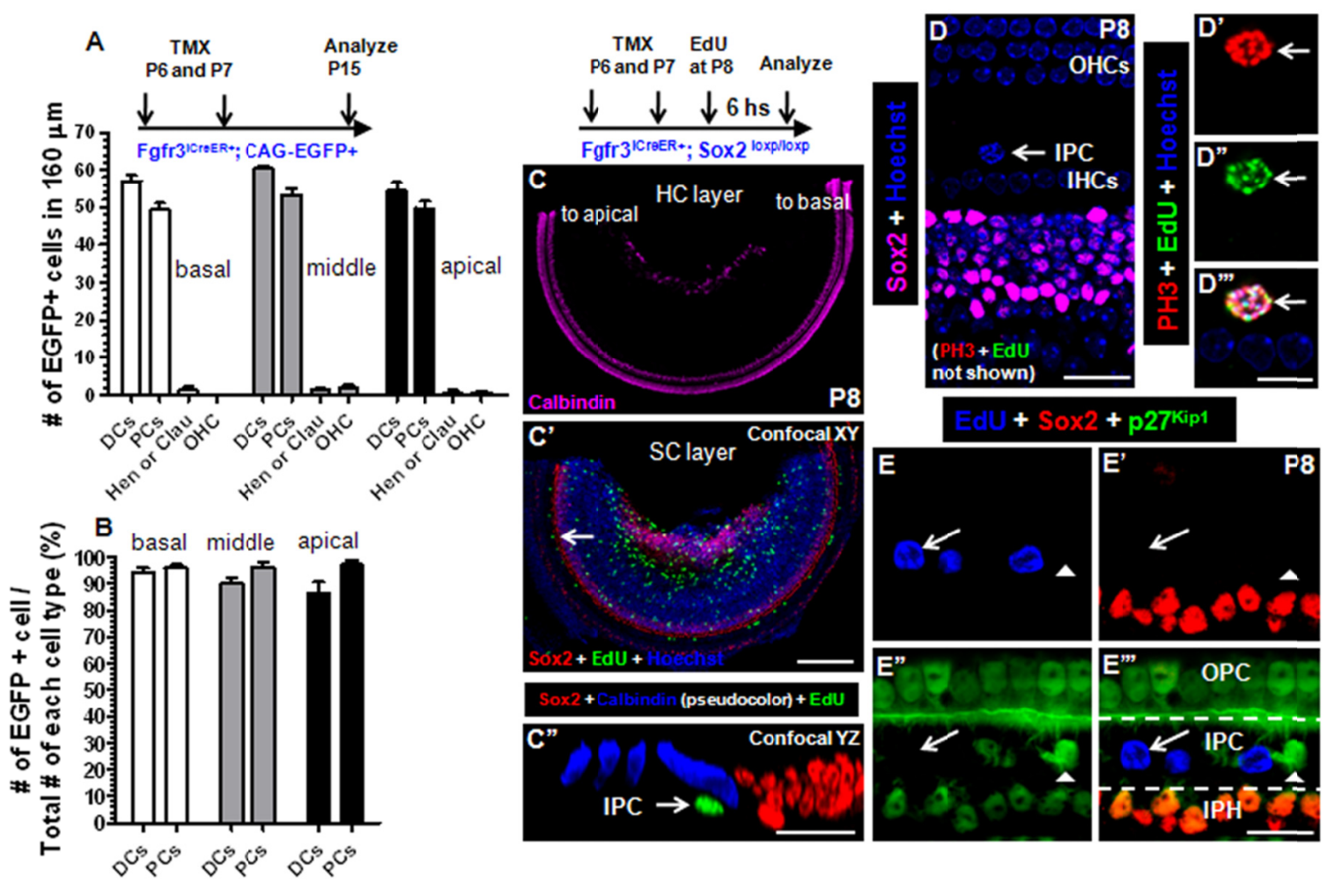

Figure 3-3 Sox2 ablation leads to proliferation of juvenile inner pillar cells.

Quantification of EGFP + cells in $\sim 160 \mu \mathrm{m}$ cochlear length. (B) Percentage of EGFP+ cells among the total number of each cell type. (C-C') Images were taken at HC layer (C) and SC layer (C'). The same EdU+ / Sox2-negative IPC is viewed in confocal XY plane (arrow in C') and YZ plane (arrow in C'). (D-D'") The PH3+ / EdU+ /

Sox2-negative IPC (arrow) migrates to HC layer. Arrows point to the same IPC. (E-E'"') Triple staining of Sox2, p27 and EdU. Arrows point to the same EdU + / Sox2-negative / p27-negative IPC. Arrow heads point to the same Sox2-negative IPC with normal p27 expression which is EdU-negative. OHCs: outer hair cells; IHCs: inner hair cells; IPC: inner pillar cell; OPC: outer pillar cell; DCs: Deiters' cells; IPH: inner phalangeal cell. Bars: $200 \mu \mathrm{m}\left(\mathrm{C}^{\prime}\right) ; 20 \mu \mathrm{m}$ (C', D, E'"); $10 \mu \mathrm{m}$ (D','). 
mice were subjected to tamoxifen at P30, EdU at P32 and were analyzed 6 hours after EdU injection. Only $5 \pm 3(n=3)$ EdU+/Sox2-negative IPCs were found across the entire cochlea (Figure 3-4B-B'), though almost all SCs have lost Sox2 expression (Figure 3-4C-C", '). Adult IPCs have lost their oblong shape, which forced us to define cells as IPCs based on their location. The limited number of EdU+ cells prompted us to speculate that adult Sox2-negative SCs might require a longer time to reenter S phase. Because adult mice can tolerate multiple EdU injections, Fgfr $3^{i C r e E R+}$; Sox $2^{\text {loxploxp }}$ mice were given tamoxifen at P30, followed by EdU injection at P34, P36, P38 and P40 and analyzed 6 hours after their last EdU injection. In each sample Sox2 staining was performed to confirm that deletion of Sox 2 did occur (data not shown). However, only $1 \pm 1(n=4)$ EdU+/Sox2-negative IPC was found across the entire cochlea (Figure 3-4D-D'). EdU+/Sox2-negative OPCs and DCs were never observed. As expected, in Fgfr $3^{i \mathrm{CreER+}}$; Sox $2^{+/+}$control mice, no EdU+ cells were observed inside the organ of Corti (data not shown). Taken together, these data suggest that adult IPCs show a very limited capacity to proliferate in the absence of Sox2.

All data described above suggest that Sox2 is a positive upstream regulator of p27. In support with this conclusion, as described in Chapter 2, deletion of p27 in neonatal PCs caused proliferation of both IPCs and OPCs. Because p27 $7^{\mathrm{Kip} 1}$ expression is maintained in adult SCs, ${ }^{65,94}$ we then determined whether acute ablation of p27 in adult (P30) PCs would also lead to a similar phenotype as Sox2 ablation in adult SCs. Because Prox 1 becomes undetectable at adult ages, ${ }^{83} \operatorname{Prox} 1^{\mathrm{CreER} /+}$ mice cannot be used. Instead we analyzed $\mathrm{Fgfr} 3^{i \mathrm{CreER}{ }^{+}} ; p 27^{\text {loxp/loxp }}$ mice that were given tamoxifen once at P30, and given EdU once at P32, P33, P34 and analyzed at P35 (Figure 3-5A-A','). We observed a very limited number of EdU+ cells $(4 \pm 2, n=3)$ (Figure 3-5B-C"',). The lack of SC specific markers does not allow us to further characterize their identities. In contrast, as internal controls, $116 \pm 20(n=3)$ EdU+ cells could be found in the lesser epithelium ridge (LER) area in the whole cochlea (arrows in Figure 3-5B).

\subsubsection{Functional consequences due to inducible loss of Sox 2 in the mouse cochlea}

Previous studies have shown that uncontrolled proliferation of cochlea SCs may result in hair cell death, ${ }^{64,65,87-89}$ which prompted us to examine the effect of inducible loss of Sox 2 on the long term health of the HCs, and the ability of the mouse to hear.

Fgfr $3^{\text {iCreER+ }} ;$ Sox $2^{\text {loxp/loxp }}$ mice were given tamoxifen at P0 and P1, EdU once at P15 and analyzed at P16 to characterize the morphology of the organ of Corti and to ask if Sox2-negative cells continue to proliferate until P15 (Figure 3-6A-B'). As expected, we found significant $\mathrm{HC}$ loss. The majority of the lost $\mathrm{HCs}$ were $\mathrm{OHCs}$, while a small fraction of them were IHCs. More HCs were lost in apical turns than middle or basal turns. Across the entire cochlea, $5 \pm 2(n=3)$ EdU+ cells were found at P16, suggesting that some Sox2-negative IPCs keep proliferating between P2 and P16 (Figure 3-6C-D). Inside the tunnel of Corti, there were supernumerary SCs (almost all were Sox2-negative) which were likely daughter cells produced from Sox2-negative IPCs (Figure 3-6E). Presence of TUNEL+ cells at P16 suggests that cell death is occurring in the area (Figure 


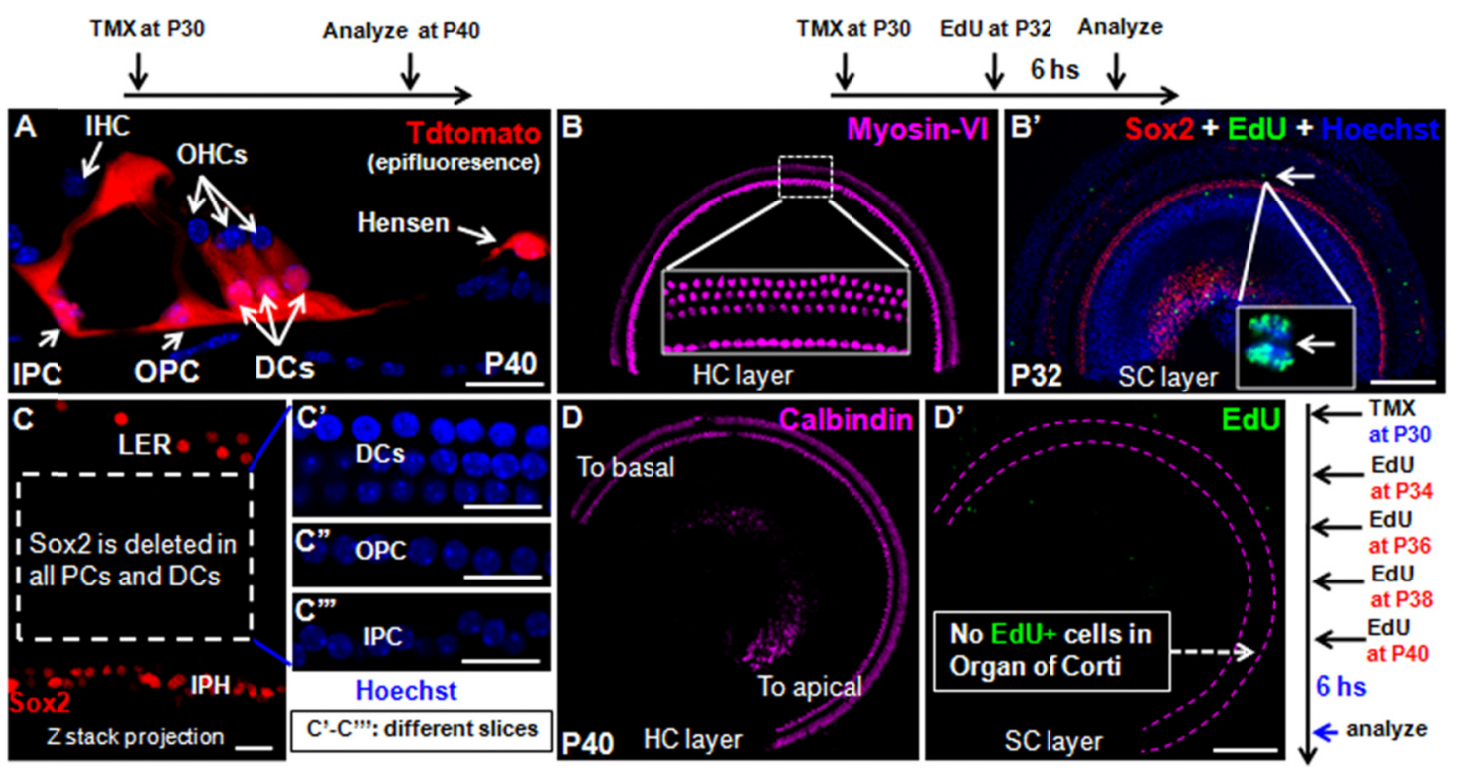

Figure 3-4 Limited proliferation of inner pillar cells (IPCs) occurs after Sox2 is ablated at adult age.

(A) Trans-section image of cochlea from $\mathrm{Fgfr} 3^{\text {iCreER+ }^{+}}$; Rosa26-CAG-Tdtomato ${ }^{\text {loxp/+ }}$ mice that were subjected to tamoxifen at P30 and analyzed at P40. (B-B') Whole mount image at HC layer (B) and SC layer (B') from $F g f r 3^{i C r e E R+}$; Sox $2^{\text {loxp/loxp }}$ mice. (C-C',') Z-stack projection image of Sox 2 staining. (D-D') Image taken from cochlear sample with multiple EdU injections at HC layer (D) and SC layer (D'). Dashed line (D') represents the organ of Corti region. OHCs: outer hair cells; IHC: inner hair cell; IPC: inner pillar cell; OPC: outer pillar cell; DCs: Deiters' cells; IPH: inner phalangeal cell; LER: lesser epithelium ridge. Bars: $20 \mu \mathrm{m}$ (A, C-C','); $200 \mu \mathrm{m}$ (B', D'). 

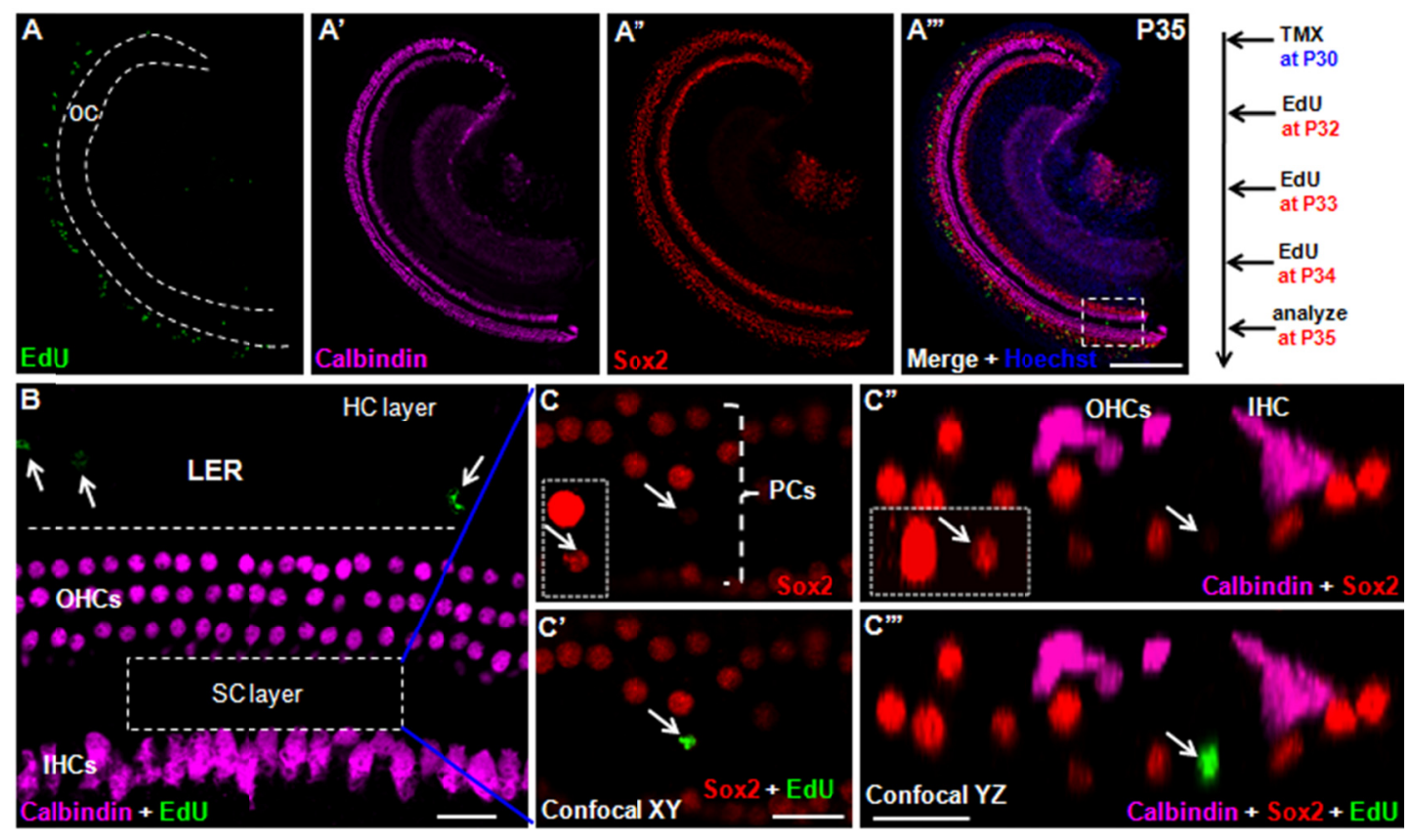

Figure 3-5 Limited proliferation of pillar cells (PCs) occurs after p27 is ablated at adult age.

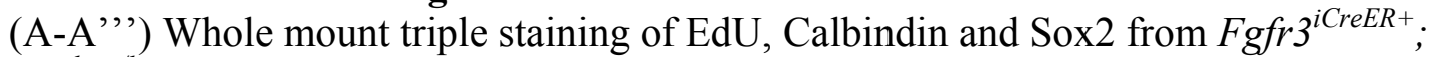
p2 $7^{\text {loxp/loxp }}$ mice. (B) High-magnified image of the square area in (A,', ). Arrows signify the EdU + cells in LER region. (C-C"') Arrows points to the same Sox2+ / EdU+ PCs viewed in confocal XY plane (C-C') and confocal $\mathrm{YZ}$ plane (C',-C','). Insets in (C) and $\left(C^{\prime}\right.$ ') are the same images taken with higher confocal laser power. IHCs: inner hair cells; OHCs: outer hair cells; PCs: pillar cells; LER: lesser epithelium ridge. Bars: $200 \mu \mathrm{m}$ (A' ', ); $20 \mu \mathrm{m}$ (B, C', C', ). 


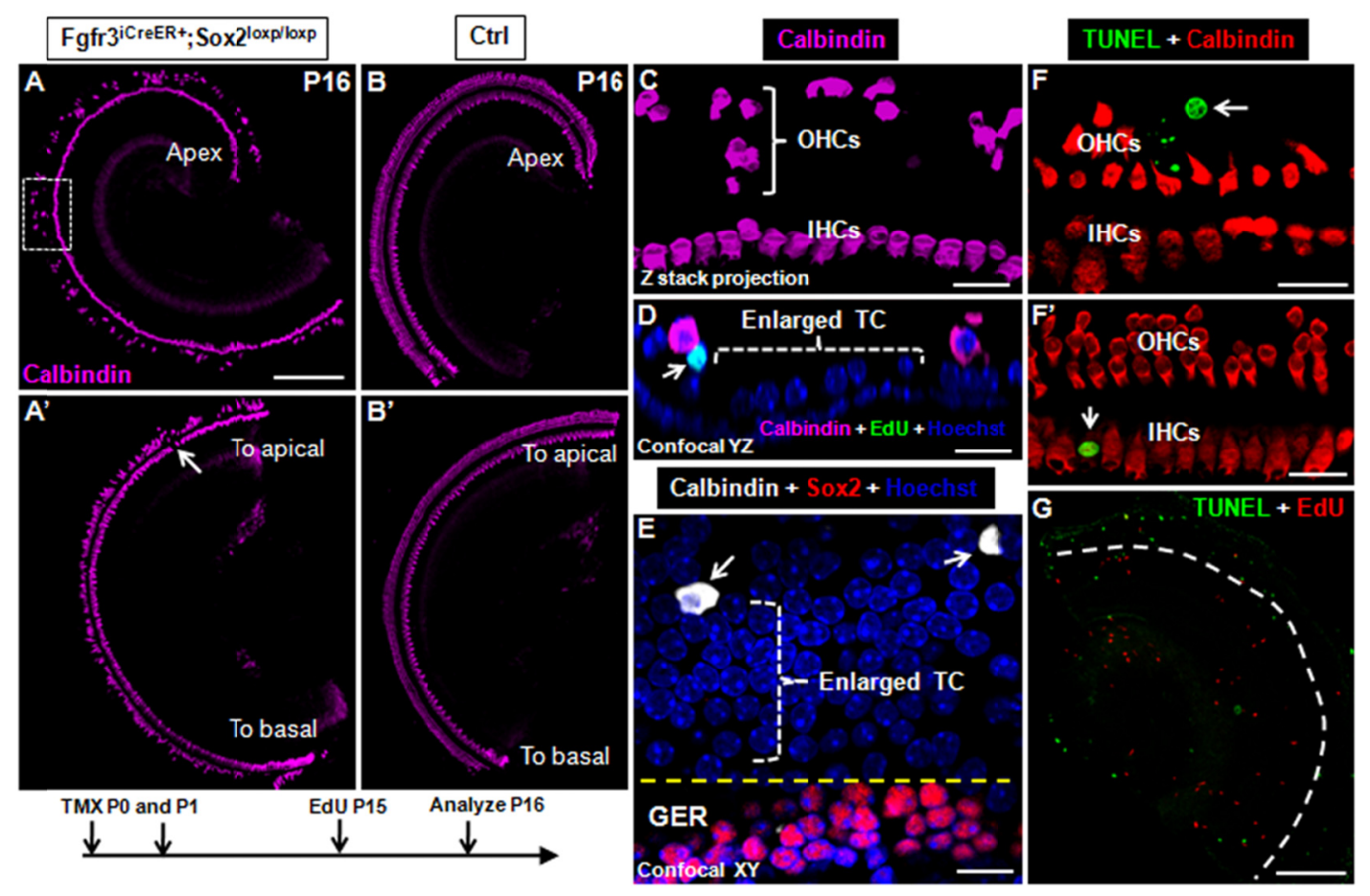

Figure 3-6 Long term effects caused by proliferation of neonatal inner pillar cells (IPCs).

(A-B') Whole mount image of Calbindin+ HCs in experimental (A, A') and ctrl (B, B') group. Arrow in (A') indicates 3 missed IHCs. (C) Confocal projection image of rectangular area in (A). (D) Image of EdU and Calbindin double-labeling viewed in confocal YZ plane. The arrow points to an EdU+ cell (likely to be daughter cells of proliferating IPCs). (E) Image of Calbindin and Sox2 double-labeling viewed in confocal $\mathrm{XY}$ plane at SC layer. Excessive Sox2-negative cells were distributed in the expanded TC. The arrows indicate endogenous HCs sinking to the SC layer. (F-F') Image of TUNEL and Calbindin double labeling. Arrows indicate dying cells. (G) Image of TUNEL and EdU double labeling. No TUNEL+ / EdU+ cells were observed. Dashed line represents the organ of Corti area. IHCs: inner hair cells; OHCs: outer hair cells; TC: tunnel of Corti. Bars: $200 \mu \mathrm{m}(\mathrm{A}) ; 20 \mu \mathrm{m}(\mathrm{C}-\mathrm{G})$. 
3-6F-F'). Absence of TUNEL+/EdU+ cells indicates that the EdU+ cells are proliferating cells, but not dying cells (Figure 3-6G).

\subsubsection{Overexpression of Sox 2 results in activation of the p27 promoter in vitro}

To understand if Sox 2 can modulate the $p 27$ promoter, we used a construct in which luciferase is driven by the $p 27$ promoter (Figure $3-7 \mathrm{~A}) .{ }^{95} \mathrm{We}$ then transfected this construct, along with an internal control of LacZ and either E2F1, Sox2 or an empty vector into different cell lines. Because Sox 2 binding has been demonstrated to be dependent on its distinct binding partners in different cell types, we chose to examine the effects of Sox 2 overexpression in three distinct cell types: Mouse Embryonic Fibroblasts (MEF), HELA, Human Embryonic Kidney Cells (HEK). When transfections were performed on all three cell types, we found that overexpression of E2F1, a known regulator of the $p 27$ promoter, resulted in up-regulation of luciferase as measured by luciferase activity. Overexpression of Sox2 led to a significant up-regulation of luciferase in both MEF and HELA cells, but had no effect in HEK cells (Figure 3-7B-D). All luciferase luminosity was normalized to beta-galactosidase luminosity in order to account for any changes due to cell viability and transfection efficiency.

\subsubsection{Endogenous levels of Sox2 bind near the p27 locus}

Recently, next generation sequencing was used to sequence all potential binding

sites of Sox 2 in the entire genome of mouse embryonic stem cells via ChIP-Seq. ${ }^{96,97}$ Both reports identified a putative Sox 2 binding site in a region downstream of the $p 27$ locus (illustrated in Figure 3-7E, chromosome 6 base pairs 134880326-134880400), as well as a potential consensus sequence for Sox 2 binding 5'-CATTGTT-3, .96 To understand if this consensus sequence is represented in the $p 27$ promoter in our construct, we performed a simple search of the $p 27$ locus (plus $2 \mathrm{~kb}$ upstream and $4 \mathrm{~kb}$ downstream) for any sequence with at least $70 \%$ homology to the published consensus sequence. We identified multiple Sox 2 consensus sites in both the 5' promoter region and the downstream region near the Sox 2 binding site identified in the ChIP-seq studies. ${ }^{96}$ The two most conserved sequences in both the 5' promoter region and the downstream region are shown in Figure 3-7E, demonstrating that the putative Sox 2 recognition sequence exists in both the promoter region and the downstream Sox 2 binding site.

However, the very limited amount of IPCs in the cochlea prevented us from demonstrating that this interaction exists in IPCs. To understand if it is possible for Sox 2 to regulate p27 we performed ChIP in MEF cells, a cell type which responded strongly to Sox 2 overexpression in the luciferase reporter assays and obtained a positive band when primers specific for the putative Sox 2 binding site were used (Figure 3-7F-G). Taken together these data suggest that not only can overexpression of Sox 2 activate the $p 27$ promoter, but endogenous Sox 2 can directly bind to its regulatory regions and control this interaction. 
A
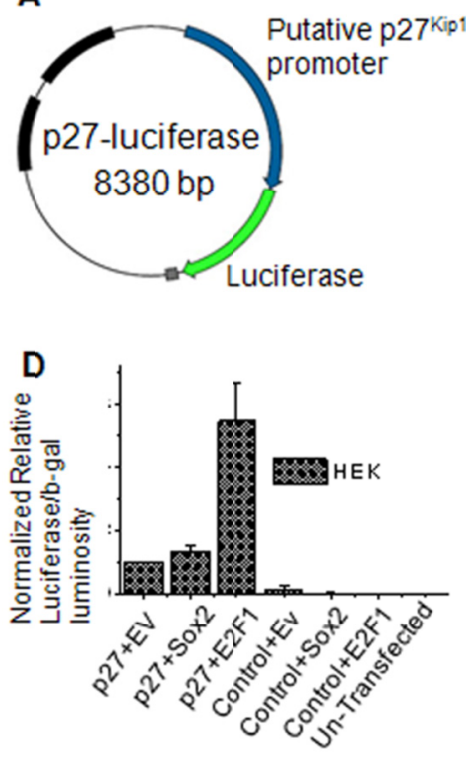

F Crosslink DNA/Protein IP for Sox2

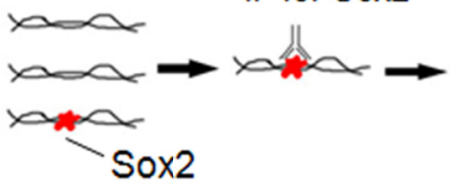

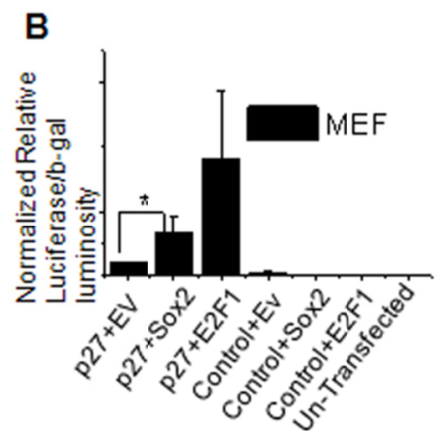
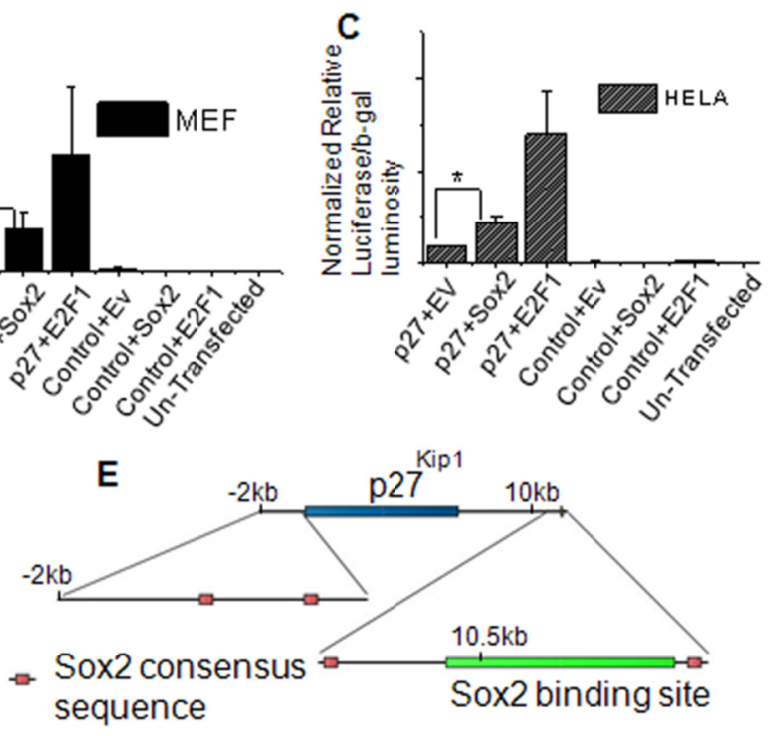

PCR for specific sequence $\sim$

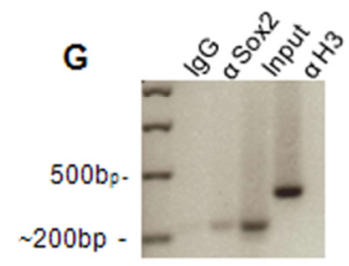

\section{Figure 3-7 Sox2 directly regulates p27 in-vitro.}

(A) Schematic of the luciferase construct utilized in the experiments. (B-D) The effect of Sox 2 overexpression on p27 transcriptional activity was measured in MEF (B), HELA (C), and HEK cells (D). All values were normalized to the negative control empty vector (Ev), then compared to the positive control E2F1, or Sox 2 overexpression. Minimal luciferase activity was detected when a promoter-less luciferase vector was used (Control), with no increases occurring in the presence of Sox 2 or E2F1. (E) Schematic of the $p 27$ locus including sites with high homology to the Sox 2 consensus sequence. (F) Schematic of the ChIP experiment utilized in this study. (G) ChIP experiments were performed in MEF cells, and a positive PCR band was obtained when a Sox 2 antibody was utilized, or total input was probed. No band was seen when rabbit IgG was used instead of the Sox 2 antibody. Positive control H3 antibody showed a band when probed with specific primers. 


\subsection{Summary and Discussion}

Here we demonstrate that Sox 2 is required to maintain the quiescent state of neonatal and juvenile IPCs. Deletion of Sox 2 led to repression of p27 and subsequent proliferation of IPCs. Deleting p27 also resulted in proliferation of IPCs, as well as OPCs; however these cells continued to express Sox2, suggesting that Sox 2 is upstream of $\mathrm{p} 27$. In addition, luciferase reporter assays and Sox2-ChIP demonstrated that Sox 2 can act as an activator of the $p 27$ promoter, and can interact with regulatory regions of the $p 27$. Together these data demonstrate that regulation of $p 27$ by Sox 2 is an important pathway involved in maintaining quiescence of IPCs. Furthermore these data clearly demonstrate the inherent heterogeneity that exists within the SC population of the organ of Corti. Only IPCs are sensitive to the Sox2-p27 repression of proliferation. Whereas OPCs need p27 but no longer Sox2, and DCs require neither proteins for cell cycle reentry. Finally this signaling cascade seems to be age-dependent because adult IPCs showed very limited response to the absence of either Sox 2 or p27.

\subsubsection{A novel role for Sox2 in postnatal cochlear supporting cells}

In many mouse embryonic tissues such as the retina, Sox 2 is highly expressed in proliferating progenitors and its loss leads to defective proliferation. ${ }^{52}$ This suggests a general role for Sox 2 to promote proliferation in embryonic tissue cells, which might explain the severe defective inner ear development in the two Sox 2 hypomorphic mouse models. ${ }^{46}$ However, by deleting Sox 2 specifically in postnatal cochlear SCs, we found the opposite and demonstrated that Sox 2 is required to keep neonatal and juvenile IPCs quiescent in part by maintaining expression of p27. Interestingly, Sox 2 is dispensable for expression of $\mathrm{p} 27$ in OPCs and DCs. Also, it is unlikely that Sox 2 deletion in postmitotic SCs would dedifferentiate them into their original progenitor state because prosensory progenitors are also Sox $2+$. This novel role for Sox 2 in regulating its target p27 may be important for other tissues and cell types.

\subsubsection{Heterogeneous effects of Sox 2 ablation in different supporting cells}

Sox 2 is robustly expressed in SCs, but loss of Sox 2 results in drastically different phenotypes in IPCs versus OPCs or DCs. To explain the different phenotypes caused by Sox 2 deletion, we need to consider our luciferase reporter experiments, which showed that different cell types respond differently to Sox 2 overexpression. We saw a lack of Sox2-p27 regulation in HEK cells, implying that this cell type does not express the appropriate co-factors for Sox 2 to regulate p27 promoter, whereas MEF and HELA cells are able to maintain this regulation. This is also in line with the current notion that Sox2 must cooperate with diverse binding partners in different cell types to efficiently drive expression of target genes. ${ }^{45}$ It is possible that like HEK cells, OPCs and DCs lack the Sox 2 binding partners needed for interaction with $p 27$, whereas IPC, MEF and HELA cells retain them, allowing for Sox2-dependent p27 expression. Alternatively, it is also possible that other Sox family members such as Sox $9^{49}$ and Sox $10^{98}$ may compensate for 
Sox 2 ablation in these cell types. Nonetheless, it will be informative in future studies to identify the potential binding partner (s) for Sox2 in IPCs. Finally, adult Sox2-negative IPCs showed limited proliferation which is in line with the general proposal that fully matured cells have declined proliferative capacity. This may also reflect a time-dependent shift away from the Sox2-p27 pathway for regulation, potentially through a shift in Sox2 binding partners available within IPCs.

\subsubsection{Heterogeneous signals of cell cycle regulation in different supporting cells}

Interestingly, different proliferative phenotypes were observed when different cell cycle inhibitors were acutely deleted in neonatal SCs. For instance when Rb was deleted in neonatal DCs and PCs, both cell types proliferated. ${ }^{73}$ As described in Chapter 2, deletion of p27 in neonatal DCs and PCs resulted in proliferation of only PCs. Deletion of Sox2 in neonatal and juvenile DCs and PCs caused proliferation in only IPCs, but not OPCs or DCs. This partial overlap of phenotypes demonstrates the importance and potential hierarchy of the cell cycle control pathway in which $\mathrm{Rb}$ is the key downstream player.

This finding is somewhat surprising in that p27 is not necessary to keep DCs quiescent because p27 is the only cell cycle inhibitor reported in postnatal DCs. ${ }^{94}$ However it is possible that DCs lack key positive cell cycle regulators, and thus do not need p27 to maintain quiescence. Consistently, cyclin D1 controls the proliferative capacity of HCs in $p 19^{--} ; p 21^{-/-}$double knockout mice, ${ }^{94}$ and only those HCs with cyclin D1 expression proliferate. Because postnatal DCs have undetectable or very low levels of cyclin D1, ${ }^{94}$ it might account for why p27-negative DCs do not proliferate. Consistently, neonatal but not adult PCs have high levels of cyclin D1, ${ }^{94}$ and p27-negative neonatal but not adult PCs do proliferate.

\subsubsection{Transient modulation of Sox 2 and p27 for mammalian hair cell regeneration}

When $\mathrm{HC}$ damage occurs in non-mammalian vertebrates such as birds, fish and amphibians, the surrounding SCs proliferate and transdifferentiate into HCs to rescue hearing capacity. When the process of $\mathrm{HC}$ regeneration is completed, SCs return to a state of quiescence again. Decreased proliferation of SCs in Phoenix mutant Zebrafish leads to defective $\mathrm{HC}$ regeneration, ${ }^{99}$ which further highlights the importance of SC proliferation in $\mathrm{HC}$ regeneration. However, mammals SCs are strictly kept quiescent even after $\mathrm{HC}$ damage occurs. This might partially account for the inability of mammals to regenerate HCs. Therefore, to restore mammals with the competence to regenerate HCs after damage, driving SCs to proliferate may be a necessary step. We have shown that the Sox2-p27 signaling pathway in IPCs and the signaling pathway involving p27 and unknown upstream regulator in OPCs are required to keep these cells quiescent. Given the high levels of both Sox2 and p27 expression in SCs, the development of transient inhibitors of either Sox 2 or p27 would allow SCs to proliferate and, in combination with local delivery, such treatment could have specific effects on inner ear cochlear SCs. 


\subsection{Future Work}

Our data clearly showed that Sox2-negative IPCs but not OPCs can proliferate. One interesting question is what are the factors that prevent Sox2-negative OPCs from proliferating. Answering this question definitively will further help to elucidate the molecular mechanisms keeping SCs quiescent at postnatal ages and provide more potential strategies to stimulate $\mathrm{SC}$ proliferation to promote $\mathrm{HC}$ regeneration. While there are many possible explanations accounting for this, one simple scenario is that some unknown factors in OPCs can compensate for the loss of Sox2. Two criteria might be used to choose such candidate factors: 1) they should be enriched, if not exclusively, in OPCs but not in IPCs; 2) as suggested by the literature, they should be able to regulate the proliferative state of SCs.

Based on the above criteria, we propose that Hes5, a Notch1 signaling target gene, could compensate for the loss of Sox 2 in neonatal and juvenile OPCs. This is supported by the two following preliminary data: First, in Hes $5^{\text {LacZ/+ }}$ knock-in mice in which the Hes 5 open reading frame is replaced by LacZ, causing that Hes5-LacZ allele is a null mutation. ${ }^{100}$ LacZ is expressed in OPCs but not in IPCs, while LacZ is also expressed in DCs (Figure 3-8A-B). Second, besides regulating the prosensory region formation and further controlling cell fate specification, Notch1 signaling (or its target genes) is also involved in cell cycle regulation of SCs around perinatal stages, as supported by the fact that excessive proliferative of SCs are observed in the Jagged2/Deltal double KO mice where Notch1 signaling is defective. ${ }^{14}$

To test this idea, we planned to analyze $F g f r 3^{\text {iCreER+ }} ; \operatorname{Sox} 2^{\text {loxp/loxp }} ; \mathrm{Hes}^{\text {LacZ/LacZ }}$ mice that will be injected with tamoxifen at P0 and P1, and analyzed at different time points. If OPCs without Sox 2 and Hes5 proliferate, it will strongly suggest that Hes5 compensates for the Sox 2 deletion in the Fgfr $3^{i \text { CreER+ }}$; Sox $2^{\text {loxp/loxp }}$ mouse model. However, one disadvantage of the $\mathrm{Fgfr} 3^{\text {iCreER+ }}$; Sox $2^{\text {loxp/loxp }}$; Hes $5^{\text {LacZ/LacZ }}$ model is that homozygous germline loss of Hes5, to some extent, causes defective embryonic cochlear development. ${ }^{28}$ Alternatively, to similarly block Hes5 function or Notch1 signaling

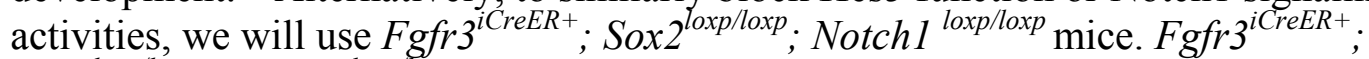
Sox $2^{\text {loxp/loxp }} ;$ Notch $1^{\text {loxp/loxp }}$ mice will be injected with tamoxifen at P0 and P1, and analyzed at different ages. Because Notch1 is the only Notch receptor expressed in mouse cochlea, ${ }^{18}$ deletion of Notch1 should block Notch activities. To guarantee that other Notch receptors will not compensate the loss of Notch1, we have analyzed $C A G^{\mathrm{CreER}{ }^{+}}$; Notch $1^{\text {loxp/loxp }} ; \mathrm{Hes}^{\text {LacZ/+ }}$ mice (experimental group) where all cells including SCs are targeted and Notch $1^{\text {loxp/loxp }} ;$ Hes $^{\text {LacZ/+ }}$ mice (control group). Both groups were injected with tamoxifen at P0 and P1, and analyzed at P7 (Figure 3-8B-C). Hes5 was used as a reporter gene for Notch activities. We found that the number of Hes5+cells were significantly decreased in $\mathrm{CAG}^{\mathrm{CreER+}} ; \mathrm{Notch} 1^{\text {loxp/loxp }} ; \mathrm{Hes} 5^{\mathrm{LacZ} /+}$ mice, relative to Notch $1^{\text {loxp/loxp }} ;$ Hes $5^{\text {LacZ/+ }}$ mice (Figure 3-8B-C). Notch $1^{\text {loxp/loxp }} ;$ Hes $5^{\text {LacZ/+ }}$ mice (Figure 3-8B-C). It further confirmed that loss of Notch1 will cause defective Notch activities in SCs. 


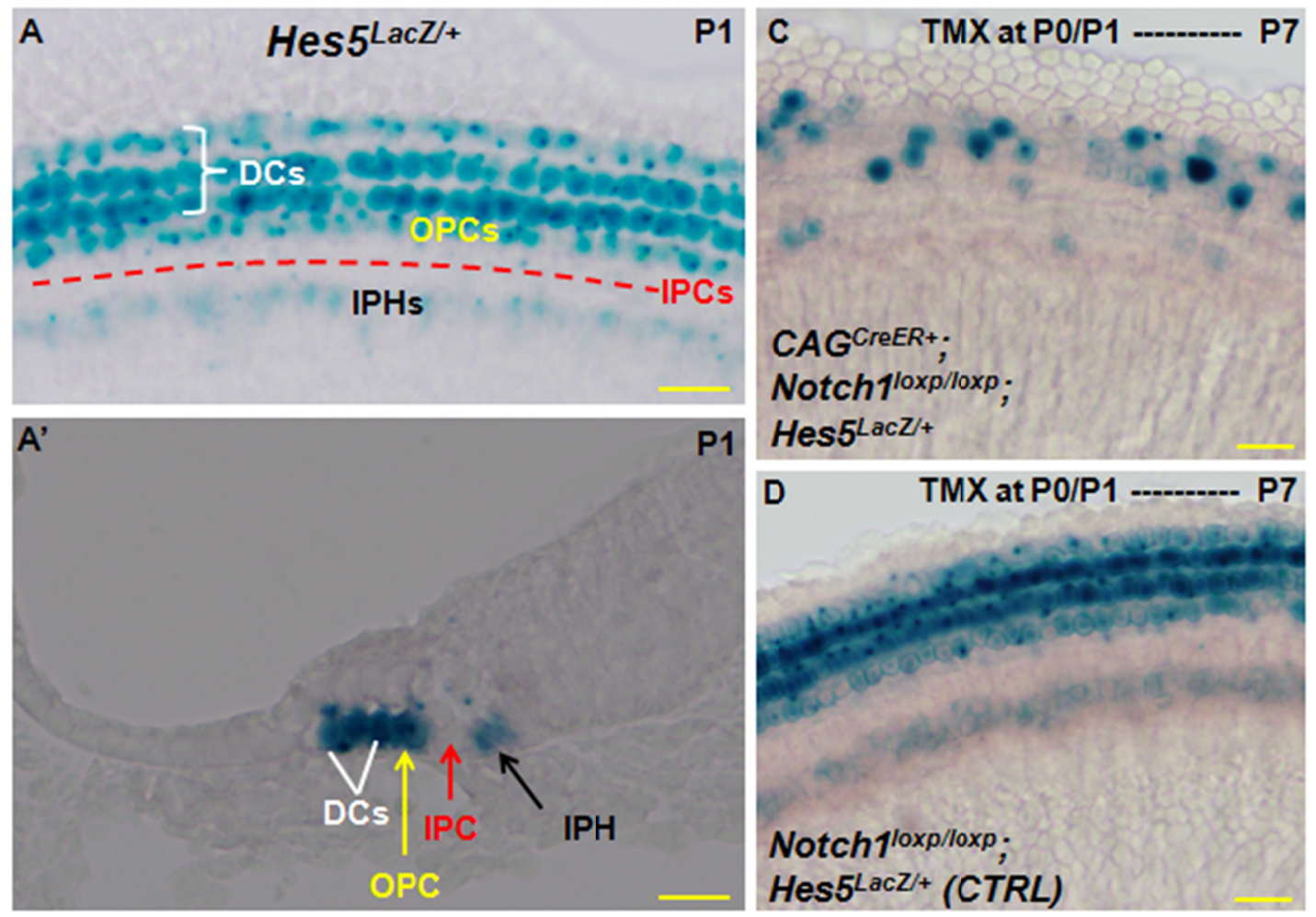

Figure 3-8 Hes5 expression pattern in wild type and Notch mutant mice.

(A-B) Whole mount (A) and trans-section image (B) of the X-gal staining of Hes $5^{\mathrm{LacZ} /+}$ mice at P1. Hes5-LacZ is expressed in DCs, OPCs and IPHs, but not IPCs. (C-D) X-gal staining of cochlear samples without Notch1 (C) and with Notch1 (D) at P7. Bars: $20 \mu \mathrm{m}$. 


\section{CHAPTER 4. OVERACTIVATION OF NOTCH 1 SIGNALING GENERATES NEW HAIR CELLS AT EARLY EMBRYONIC AGES}

\subsection{Introduction}

The otocyst derived cochlear cells roughly can be divided into three groups, 1) sensory HCs and SCs inside the organ of Corti; 2) non-sensory cells in LER or GER regions; 3) the cochlear spiral ganglion neurons (SGNs). There is a belief that mouse $\mathrm{HCs}$, SCs and SGNs originate from the same early common neural-sensory progenitor cells, mainly based on studies of the chick inner ear development. ${ }^{101}$ Recently, such a model has been further supported but it has not been definitively proven by mouse inner ear development studies. ${ }^{102,103}$ These neural-sensory progenitor cells further commit into two subgroups, prosensory progenitors that develop into HCs or SCs, and neural progenitor cells that differentiate into SGNs.

Morphogenesis of cochlear HCs is a complex but step-by-step process. Notch1 signaling is able to specify progenitor cell populations among cochlear cells. Therefore, it is interesting to test whether overactivation of Notch1 signaling in cochlear non-sensory cells can convert them into progenitor cells which are further permissive to become $\mathrm{HCs}$ or SCs, by using genetic mutant mouse models.

To induce ectopic Notch1 signaling independently of Notch1 receptor proteolysis, a genetic knock-in mouse model has been generated where the Notch1 intracellular domain (NICD) is driven by a ubiquitously active Rosa26 promoter. ${ }^{104}$ However, there is a "stop" sequence flanked by two loxP sequences in the same orientation, thus upon Cre-mediated deletion of the "stop" sequence, NICD transcription is induced. To induce ectopic NICD expression in non-sensory cells, we used a $C A G^{\text {CreER+ }}$ mouse line in which tamoxifen inducible Cre activity is ubiquitously expressed.

\subsection{Materials and Methods}

\subsubsection{Mouse strains}

$$
\text { Rosa26-NICD }{ }^{\text {loxp } /+}, \text { Notchl }^{\text {Cre (low) /+ }} \text {, Rosa26-EYFP }{ }^{\text {loxp } /+} \text { and }
$$

Rosa26-CAG-Tdtomato ${ }^{\text {loxp } /+}$ were purchased from Jackson Laboratory (USA). The $C A G^{\text {CreER+ }}$ mouse line was obtained from Dr. Guillermo Oliver (St. Jude Children's Research Hospital) with permission from Dr. Andrew McMahon (Harvard University). Mice were crossed at $5 \mathrm{pm}$ and the next morning was designated as E0.5 when vaginal plugs were found. Pregnant female mice were given tamoxifen (intraperitoneal, $100 \mu \mathrm{g} / \mathrm{g}$ body weight) once when embryos were at $\sim \mathrm{E} 10.5, \sim \mathrm{E} 13 .{ }^{105}$ All animal work conducted in this study was approved by the Institutional Animal Care and Use Committee at St. Jude Children's Research Hospital and performed according to NIH guidelines. 


\subsubsection{Histology and immunofluorescence}

Detailed histological procedures have been described in Chapter 2. Cochlear samples of the inner ear were processed by our routine protocols described previously. All images were examined using a Zeiss LSM 700 confocal microscope. The following primary antibodies were used: anti-Myosin-VI (rabbit, 1:200, 25-6791, Proteus Bioscience, Ramona, CA), anti-Sox10 (goat, 1:250, sc-17342, Santa Cruz Biotechnology), anti-TUJ1 (mouse, 1:1,000, MMS-435P, Covance, Princeton, NJ), anti-GFP (chicken, 1:1000, ab13970, Abcam, Cambridge, UK), The following secondary antibodies were used: donkey anti-rabbit Alexa Fluor 647 (1:1000, A31573, Invitrogen), donkey anti-chicken DyLt 488 (1:200, 703-486-155, Jackson ImmunoResearch, West Grove, PA), donkey anti-goat Alexa Fluor 568 (1:1000, A11057, Invitrogen), goat anti-rabbit Alexa Fluor 568 (1:1000, A11036, Invitrogen), goat anti-mouse Alexa Fluor 647 (1:1000, A21236, Invitrogen) and goat anti-chicken Alexa Fluor 488 (1:1000, A11039, Invitrogen).

\subsubsection{Cell counting}

The entire cochlear was carefully divided into basal, middle and apical turns. Using the preliminary low magnification confocal image, the length of each turn was first measured by drawing a line in the middle of the outer hair cells (OHCs) and the inner hair cells (IHCs). By defining the basal hook part as $0 \%$ and the most apex portion as $100 \%$, the $25 \%, 50 \%$, and $75 \%$ areas were used to represent basal, middle and apical turns, respectively. Confocal $\mathrm{Z}$ stack scanning was then performed at $1 \mu \mathrm{m}$ intervals.

\subsubsection{Statistical analyses}

All data were expressed as mean \pm SEM. Cell counts were compared by a one-way ANOVA, followed by a Student's t test with a Bonferroni correction. GraphPad Prism 5.0 was used for all statistical analyses.

\subsection{Results}

\subsubsection{Constitutive overactivation of NICD in otocyst cells generates hair cells}

$C A G^{\text {CreER+ }}$ mice were bred with Rosa26-NICD ${ }^{\text {loxp } /+}$ mice to get the $C A G^{C r e E R+}$; Rosa26-NICD ${ }^{\text {loxp/+ }}$ as experimental groups, and $C A G^{\text {CreER- }}$; Rosa26-NICD ${ }^{\text {loxp } /+}$ as control groups. In this study, cochlear samples were treated with tamoxifen at different developmental stages. The earliest tamoxifen injection was at $\sim \mathrm{E} 10.5$, because it was difficult to obtain live mutant embryos at perinatal ages when the pregnant mother was given tamoxifen at $\sim$ E9.5 or earlier. After Cre-mediated recombination, NICD and EGFP transcription are coupled to the same Rosa26 promoter. Thus, EGFP should faithfully 
reflect NICD expression. Although the $C A G$ promoter is ubiquitously active, with a single tamoxifen injection the mice can be considered mosaic, similar to chimeric mice in which wild-type and mutant cells (with ectopic NICD) are randomly mixed together. Because tamoxifen frequently caused dystocia, we analyzed embryos at $\sim$ E19 (equivalent to P0 in most cases).

Ectopic sensory areas containing Myosin-VI+/EGFP- HCs were also found in the cochlea (Figure 4-1). There are three regions with ectopic HCs. The first was the cochlear spiral ganglion (SGN) area (Figure 4-1G-G', and Figure 4-1K-L'). Among the EGFP+ patches $(15 \pm 5, n=4)$, approximately half contained variable numbers $(7 \pm 2)$ of new HCs. Interestingly, patches with new HCs were larger than those without new HCs. The second region was the endogenous organ of Corti. An expanded organ of Corti with supernumerary HCs was found in the basal or apical turn $(n=4)$ (Figure $4-1 \mathrm{H}-\mathrm{H}$ ') $)$. The third region was lesser epithelium ridge (LER) where ectopic clustered HCs $(6 \pm 4, n=4)$ were observed (Figure 4-1J). In all three areas, cells (either EGFP+ or EGFP-) surrounding these new HCs expressed Sox10, a SC marker (Figure 4-2). Notably, the greater epithelial ridge (GER) areas $(n=4)$, which contained many EGFP+ cells, had no Myosin-VI+ HCs (Figure 4-1H-I). It is consistent with another recent report where different chick inner ear cells respond differently to overactivation of Jagged $1 .{ }^{106}$ No abnormal phenotype was found in control $C A G^{\text {CreER - }}$; Rosa26-NICD ${ }^{\text {loxp } /+}$ embryos subjected to tamoxifen at $\sim \mathrm{E} 10.5$ ( $n=3$, data not shown).

\subsubsection{Cellular responsiveness to ectopic NICD declines during mouse cochlear development}

Next, $C A G^{C r e E R+}$; Rosa26-NICD ${ }^{\text {loxp/+ }}$ embryos were treated with tamoxifen once at $\sim$ E13. Although numerous EGFP + cells were found in cochleae $(n=4)$, no ectopic HCs were detected (Figure 4-3).

To confirm the age-declined response of cochlear cells to ectopic NICD expression, neonatal $C A G^{C r e E R+} ;$ Rosa26-NICD ${ }^{\text {loxp/+ }}$ mice were further treated with tamoxifen at P0 and P1, and analyzed at P10. However, the tracer EGFP became faint and difficult to visualize at P10, possibly because of decreased Rosa26 promoter activity and IRES-mediated EGFP translation. Similar difficulties have been encountered in older retina cells. ${ }^{107}$ Therefore, to better visualize cells with Cre-mediated recombination, we crossed $C A G^{C r e E R+}$; Rosa26-NICD ${ }^{\text {loxp/loxp }}$ with Rosa26-EYFP ${ }^{\text {loxp/loxp }}$ to get $C A G^{C r e E R^{+}}$; Rosa2 $6^{\text {EYFP/NICD }}$ mice as the experimental group, and $C A G^{\text {CreER - }} ;$ Rosa $26^{\text {EYFP/NICD }}$ littermates as controls. EYFP was used to visualize most of the cells with ectopic NICD. Note that, because EYFP and NICD were not strictly coupled to the same Rosa26 locus promoter, there were some EYFP+ cells that might not have ectopic NICD expression or some EYFP-negative cells that indeed expressed ectopic NICD. $C A G^{\mathrm{CrER}+}$; Rosa2 $6^{E Y F P / N I C D}$ mice were treated with tamoxifen at P0 and P1, and analyzed at P10. Similar to Figure 4-3, many EYFP+ cells were observed, but again no new HCs were identified (data not shown). 


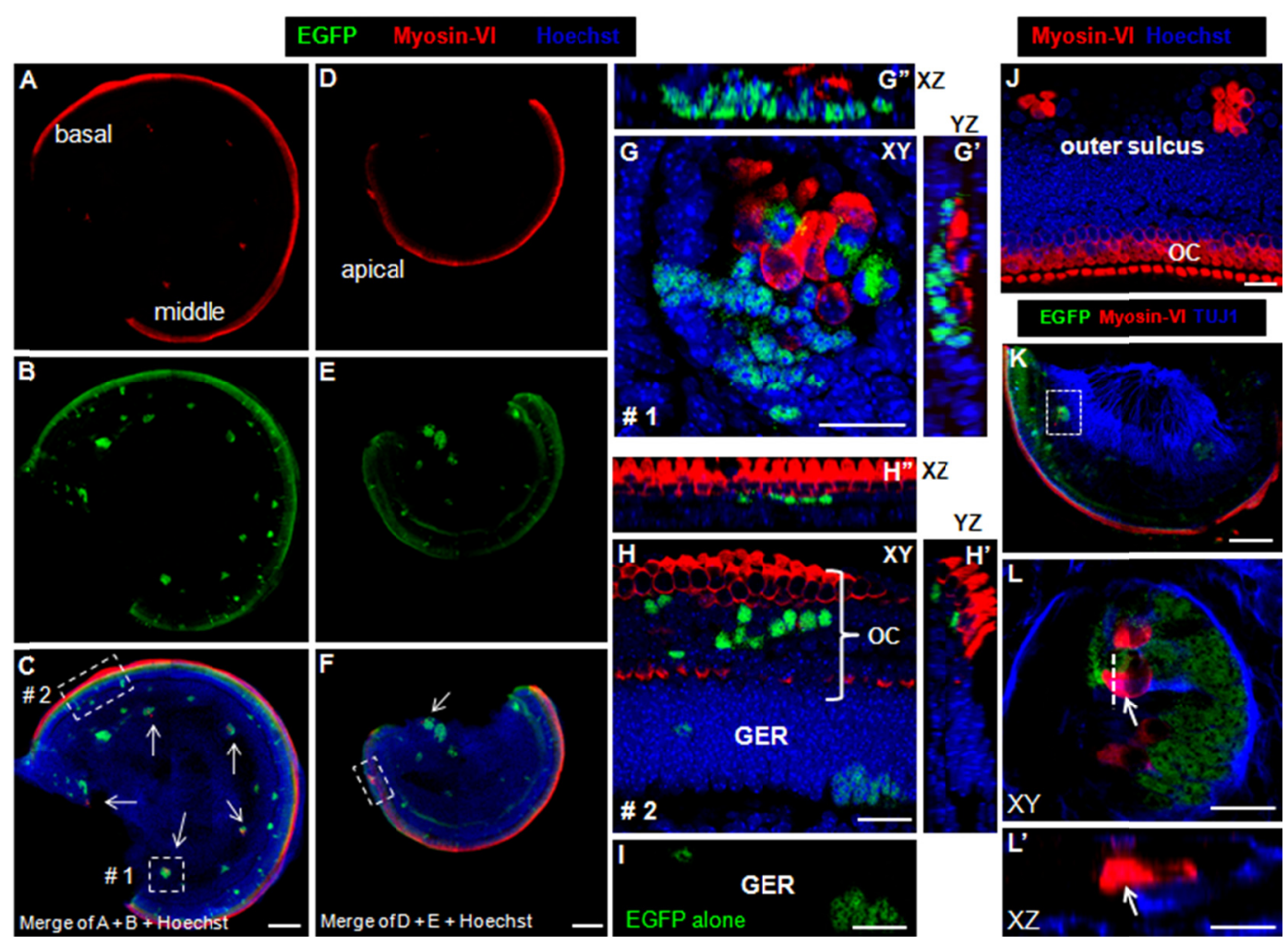

Figure 4-1 Ectopic HCs in the cochlea with constitutive Notch1 signaling. (A-F) Images of the cochlea taken from embryos of $C A G^{\text {CreER+ }}$; Rosa26-NICD ${ }^{\text {loxp } /+}$ treated with tamoxifen at $\sim$ E10.5 and analyzed at $\sim$ E19. White arrows in $(C, F)$ point to ectopic sensory epithelia with new HCs in the spiral ganglion area. Dotted rectangular areas in (C, F) mark the expanded organ of Corti. (G-G') Confocal three-dimensional, high-magnification images of an ectopic sensory patch (\#1 in C). (H-H' ') Highmagnification images of an expanded organ of Corti (\#2 in C). Extra rows of outer hair cells were present and were EGFP-negative, whereas some, but not all, SCs were EGFP+. (I) The same GER area shown in $(\mathrm{H})$, where no new HCs were observed. (J) Presence of extra HCs in the outer sulcus area. (K-L') Triple staining of TUJ1, Myosin-VI and EGFP. (L) High-magnification image of the square area in $(\mathrm{K})$. (L') Image of the confocal XZ plane through the dashed line in (L). Arrows target the same ectopic HC. OC: organ of Corti. GER: greater epithelium ridge. Scale bars: $200 \mu \mathrm{m}$ in $(\mathrm{C}-\mathrm{F}, \mathrm{K})$ and $20 \mu \mathrm{m}$ in (G-I, J, L-L'). 


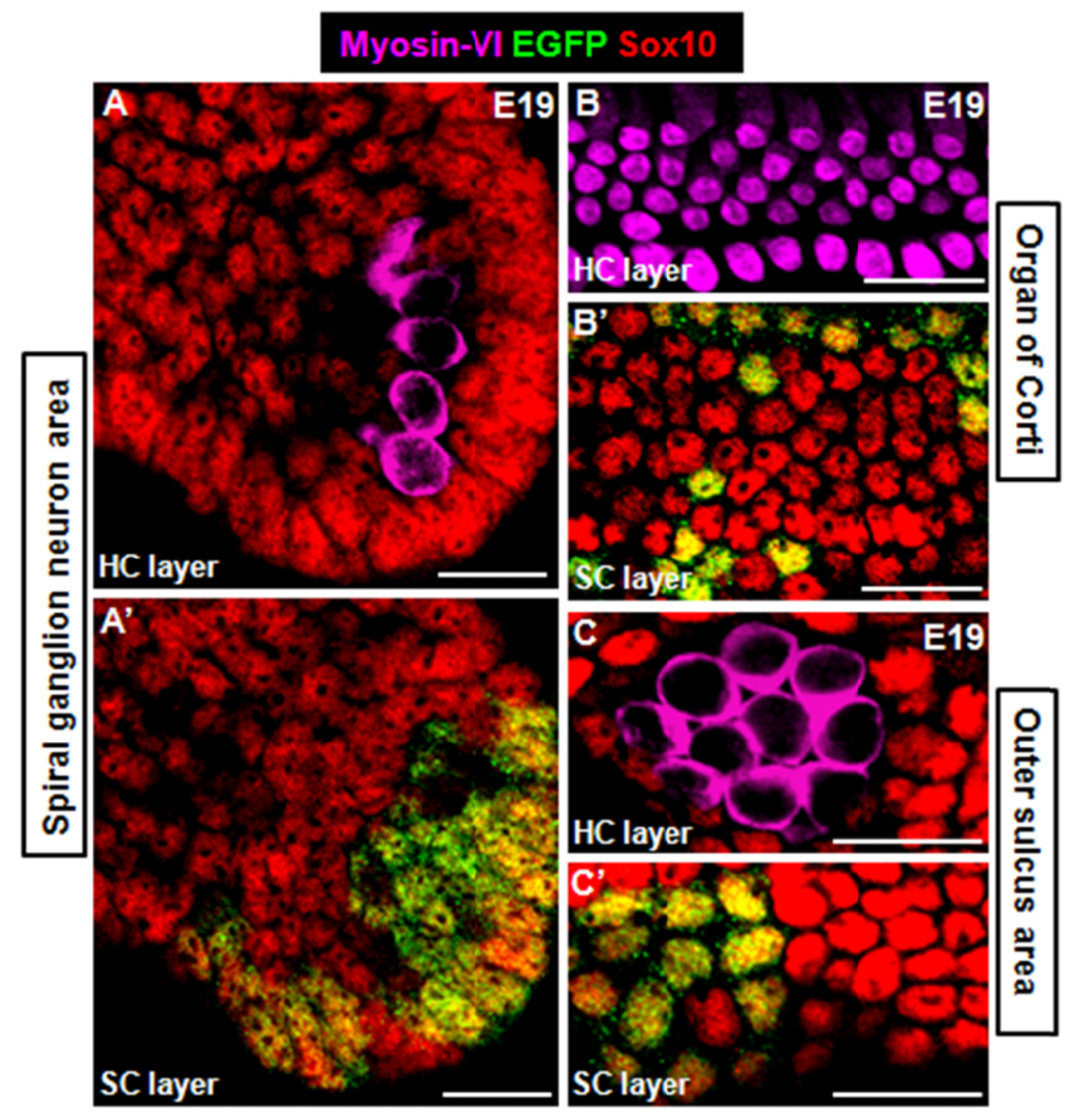

Figure 4-2 Expression pattern of Sox10 in ectopic cochlear SCs.

Triple staining of Myosin-VI, Sox 10 and EGFP of cochlear samples from $\mathrm{CAG}^{\mathrm{CreER}{ }^{+} \text {; }}$ Rosa26-NICD ${ }^{\text {loxp } /+}$ given tamoxifen at $\sim$ E10.5 and analyzed at $\sim$ E19. Ectopic HCs (Myosin-VI+) were Sox10- and SCs (EGFP+ or EGFP-) were Sox 10+ in spiral ganglion neuron area (A-A'), organ of Corti (B-B') and outer sulcus area (C-C'). Scale bars: 20 $\mu \mathrm{m}$. 


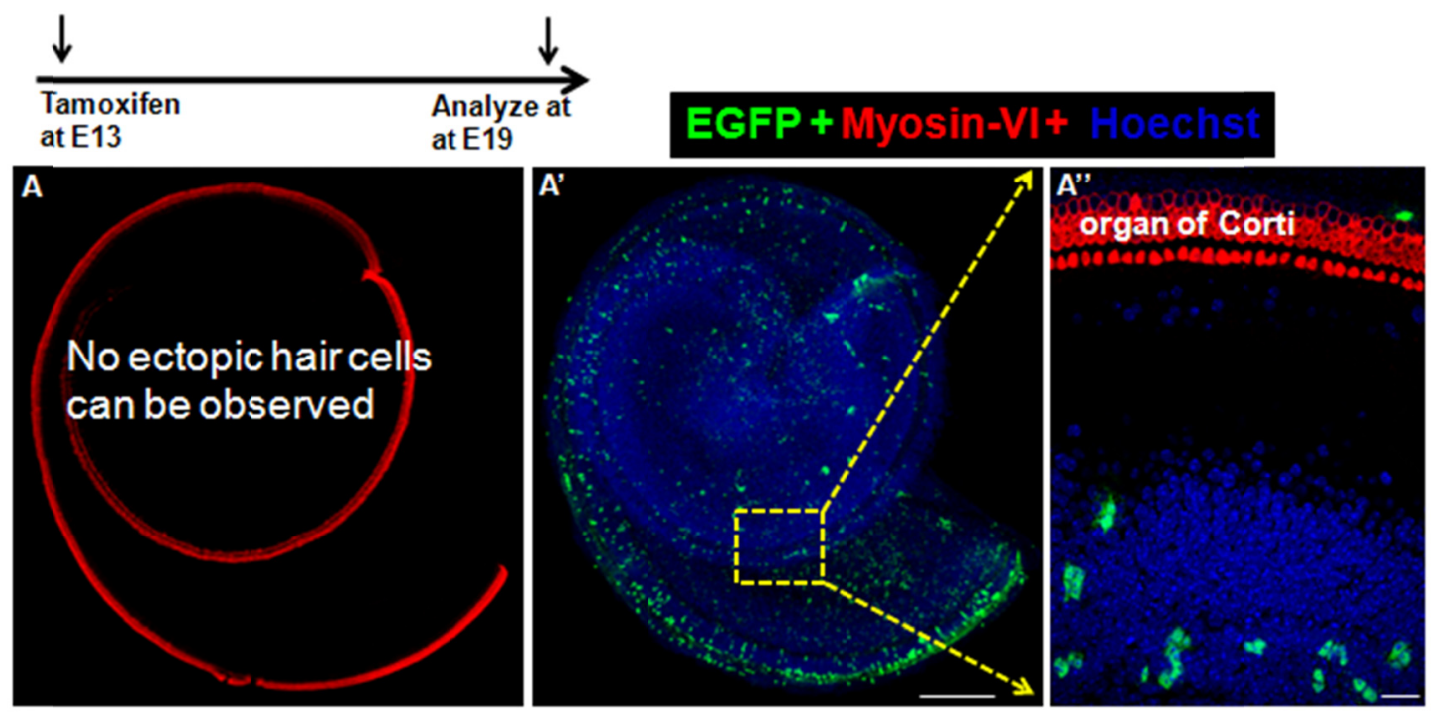

Figure 4-3 Declined response of older embryonic cochlear cells to ectopic NICD expression.

(A-A') Images of the cochlea taken from embryos of $C A G^{C r e E R+} ;$ Rosa26-NICD ${ }^{\text {loxp }}{ }^{+}$that were treated with tamoxifen at E13 and analyzed at E19. While there were many EGFP+ cells everywhere in the cochleae, ectopic hair cells were no longer observed. (A") High magnification image of the yellow square region in ( $\left.A^{\prime}\right)$. Inside the organ of Corti lied the normal three rows of outer hair cells and one row of inner hair cells. Bars: $200 \mu \mathrm{m}\left(\mathrm{A}^{\prime}\right)$ and $20 \mu \mathrm{m}\left(\mathrm{A}^{\prime \prime}\right)$. 


\subsubsection{Almost all cochlear progenitors experiencing Notch1 signaling commit to supporting cell fate in normal development}

Our data suggest that, although NICD overactivation can specify ectopic sensory patches or progenitors, cells with constitutive NICD exclusively develop into SCs. To further understand whether it is also true in normal cochlear development, we planned to perform genetic fate mapping analysis of the cochlear cells experiencing Notch1 signaling.

We crossed the Notch1 $1^{\text {Cre (Low)/+ }}$ mouse line where Notch1 intracellular domain (NICD) was replaced by $6 \times \mathrm{Myc}-$ tagged Cre recombinase with the nuclear localization signal (NLS), resulting in a null mutation of Notch $1 .{ }^{108}$ Heterozygous mice are fertile and viable whereas homozygous mice die at $\sim \mathrm{E} 9.5$, which is consistent with results of two Notchl knockout mouse lines previously characterized. ${ }^{109,110}$ In Notch $1^{\text {Cre }(L o w) /+}$ mouse, binding of Notch ligands to the intact extracellular domain of the Notch1 receptor triggers proteolytic cleavage of the Notch1 receptor transmembrane domain, release of Cre from the plasma membrane, and subsequent translocation of Cre into nuclei. When Notch ${ }^{\text {Cre (Low)/4 }}$ mice are crossed with conditional Rosa26-CAG-Tdtomato ${ }^{\text {loxp } /+}$ mice, cells with Notch1 activity and their descendants can be permanently traced with red fluorescent protein Tdtomato in Notch ${ }^{\text {Cre }(\text { Low }) /+} ;$ Rosa26-CAG-Tdtomato ${ }^{\text {loxp/+ }}$ mice (Figure 4-4).

Because the cell fate commitment is completed after birth, we characterized the Notch $1^{\text {Cre (Low)/+ }}$; Rosa26-CAG-Tdtomato ${ }^{\text {loxp } /+}$ at P6. In each cochlea $(n=3)$, we quantified the Tdtomato + cells inside the organ of Corti, which include SCs (PCs and DCs) and HCs. Along the entire cochlea, only $10 \pm 2(n=3) \mathrm{HCs}$ were Tdtomato+ occupying $\sim 0.4 \%$ of the total Tdtomato + cells, while $3075 \pm 100(n=3)$ were SCs, occupying $\sim 99.6 \%$ of the total Tdtomato+ cells (Figure 4-4H). It further proves that during cochlear development, almost all cochlear progenitors experiencing Notch1 signaling develop into SCs but not $\mathrm{HCs}$, and suggests that Notch1 signaling blocks the $\mathrm{HC}$ fate commitment and/or further differentiation. ${ }^{48}$

\subsection{Summary and Discussion}

Our data suggests that the potential of Notch1 signaling in specifying sensory patches is transient and declines with maturation, which highlights the need for additional signals (i.e. Atoh1 overactivation in Chapter 5) for $\mathrm{HC}$ regeneration in mammals after birth.

\subsubsection{Lateral induction and lateral inhibition effects of Notch1 signaling}

Notch1 signaling elicits lateral induction effects at early embryonic ages (E12 E14.5) when prosensory progenitors are being specified. At later embryonic ages (after E14.5), Notch1 signaling induces lateral inhibition effects when HC and SC 


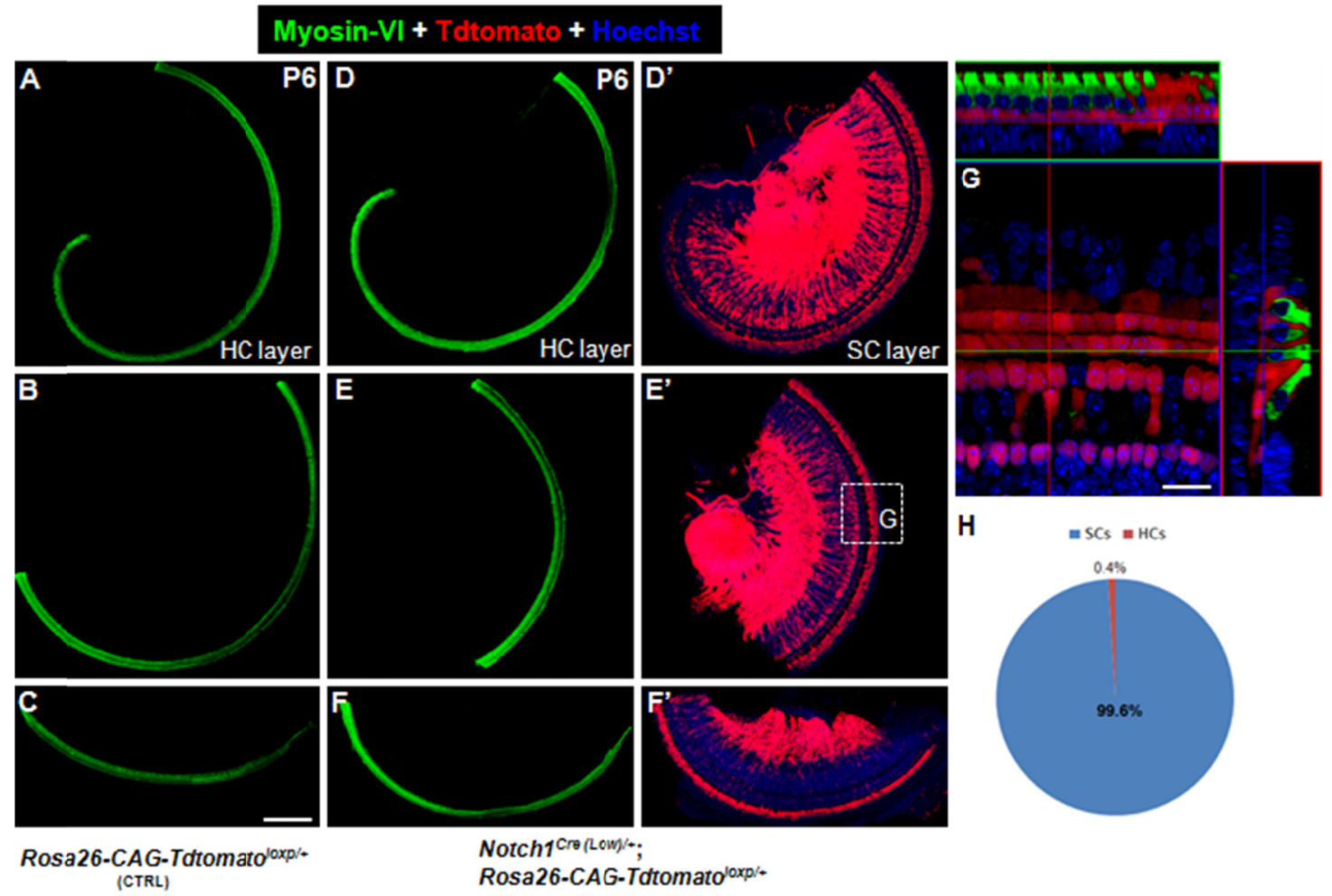

Figure 4-4 Genetic fate mapping analysis of cells experiencing Notch1 signaling. (A-C) Myosin-VI immunostaining of cochlear samples from Rosa26-CAG-Tdtomato ${ }^{\text {loxp } /+}$ mice at $\mathrm{P} 6$ in apical (A), middle (B) and basal (C) turn. No Tdtomato+ cells can be found in the cochlea. (D-F') Myosin-VI immunostaining and epifluorescent Tdtomato of samples dissected from Notch1 ${ }^{\text {Cre }(\text { Low }) /+}$; Rosa26-CAG-Tdtomato ${ }^{\text {loxp } /+}$ at P6 in apical (D, D'), middle (E, E') and basal (F, F') turn. $(\mathrm{G})$ is the confocal 3-D high magnification image of the white square region in $\left(\mathrm{E}^{\prime}\right) .(\mathrm{H})$ Percentage of Tdtomato+ cells. SCs occupied $99.6 \%$, while HCs only did $0.4 \%$. 
differentiation starts. The phenotypes observed in our $C A G^{\text {CreER+ }}$; Rosa26-NICD ${ }^{\text {loxp/+ }}$ model can be explained by these two sequential but different effects. EGFP and NICD share the same cis-transcription element. Therefore, we defined NICD+ cells as EGFP+ cells and NICD - cells as EGFP- cells. Such a definition has been used in previous studies in other systems involving this Rosa26-NICD ${ }^{\text {loxp/4 }}$ strain. ${ }^{13,15,107}$

Given the permissive cellular environment, NICD overactivation at $\sim \mathrm{E} 10.5$ induced new HCs in the EGFP+ patches. All new HCs were EGFP-, and some SCs were $\mathrm{EGFP}+$. While the emergence of EGFP + SCs can easily be explained by the prosensory promoting ability of Notch1 signaling, new EGFP- HCs and SCs should be generated by the communication between EGFP+ cells and EGFP- cells, known as the lateral induction effects of Notch1 signaling. In other words, single tamoxifen injection first transforms some non-sensory cells into prosensory progenitors (EGFP+). These EGFP+ prosensory progenitors in turn further trigger their neighboring non-sensory cells, which are not targeted by tamoxifen and are EGFP-, to turn on Notch1 signaling. It eventually leads to the transformation of these EGFP - cells into prosensory progenitors. Thus, with EGFP as a lineage tracer, new prosensory progenitors can be divided into EGFP + and EGFP-groups. EGFP- progenitors can differentiate into HCs or SCs. The final cell fate of each EGFP- progenitor might be mediated by Notch1 signaling among different EGFP- progenitors (here NICD from the endogenous Notch1 but not Rosa26 locus might be involved), referred to as lateral inhibition between HCs and SCs. In contrast, EGFP+ progenitors were prevented from committing into HCs because they constitutively expressed NICD (at the Rosa26 locus), and hence EGFP+ progenitors exclusively developed into SCs.

\subsubsection{Roles of Notch1 signaling in development of the cochlear neuronal lineage}

Mouse inner ear neural and sensory progenitors are believed to originate from the Ngn1+ neural-sensory progenitors. ${ }^{101}$ This hypothesis, especially in the inner ear vestibular part, is further supported by the lineage tracing study with $\mathrm{Ngnl}^{\mathrm{CreER+}} ; \mathrm{Z} / \mathrm{EG}$ mice $^{102}$ and another study reporting that deletion of NeuroD1 leads to ectopic HCs in vestibular ganglia. ${ }^{103}$ However, despite cochlear SGNs being traced in $\mathrm{Ngnl}^{\mathrm{CreER+}} ; \mathrm{Z} / \mathrm{EG}$ mice, cochlear prosensory progenitors were not, raising the question that whether cochlear SGNs and prosensory progenitors derive from the same neural-sensory progenitors.

The observation that delaminating neuroblasts (neural progenitors) express the Notch ligand Delta $1^{9}$ supports the idea that sensory progenitors are gradually specified among the neural-sensory progenitors by Notch1 signaling. In our study, presence of ectopic HCs in cochlear SGN regions of $\mathrm{CAG}^{\mathrm{CreER+}}$; Rosa26-NICD ${ }^{\text {loxp/+ }}$ embryos suggests that cochlear SGN progenitors with ectopic NICD are converted to prosensory progenitors, which can further differentiate into either HCs or SCs, even though we cannot rule out the possibility that some of the new HCs might originate from glia cells. In addition, defective Notch1 signaling by deleting Delta1 resulted in expanded neural 
regions. ${ }^{16}$ Taken together, these results suggest that cochlear sensory progenitors and SGN progenitors originate from identical neural-sensory progenitors.

\subsubsection{Different dosages of Notch1 signaling activity during cochlear development}

Series of studies have suggested that Notch1 signaling is induced in progenitors at early stage of cochlear development. An interesting question is why only $0.4 \%$ of HCs were traced with Tdtomato in our fate mapping analysis of Notch $1^{\text {Cre (Low)/+ }}$; Rosa26-CAG-Tdtomato ${ }^{\text {loxp } /+}$ mice (Figure 4-4). There are at least two potential explanations; one is that SCs and HCs are indeed from two different progenitor pools that, however, are difficult to distinguish. The second is due to the different dosages of Notch1 expression levels (or Notch1 signaling) between the early (lateral induction stage) and late developmental stages (lateral inhibition). It is possible that Notch1 expression level in cochlear progenitors is too low to elicit efficient Cre-mediated recombination. However, Notch1 expression is upregulated in progenitors committing to SCs and efficient Cre-mediated recombination occurs. The different dosages of Notch1 expression is now further supported by another recent report where Notch1 signaling is shown not necessary to specify cochlear progenitors. ${ }^{111}$

\subsubsection{Comparison of three different models explaining the induction of ectopic hair cells in mice}

Besides our $C A G^{C r e E R+}$; Rosa26-NICD ${ }^{\text {loxp/+ }}$ model, two other mouse genetic models have been recently used to show that overactivation of NICD can induce the generation of ectopic HCs. ${ }^{13,15}$ These three models complement each other to provide evidence of Notch1 signaling activities in the developing mouse inner ear.

The advantage of the Pan et al. mode ${ }^{13}$ is that it combines the mouse Cre/loxp and Tet-On genetic systems. ${ }^{12}$ NICD is transiently overactivated so that only lateral induction of Notch1 signaling is augmented and subsequent lateral inhibition is intact. Thus, ectopic prosensory progenitors can become either HCs or SCs. This model is different from our model in the following ways: 1) ectopic HCs can be found in cochlear SGNs regions across the entire turns in our model (Figure 4-1) but exclusively in basal turns in the Pan et al. model; 2) ectopic HCs can be found in the cochlear ventral part in our model (Figure 4-1) but in the dorsal part only in the Pan et al. model; 3) our data show that not all cells ( $\sim$ E10.5) respond to NICD and become prosensory progenitors, but all cells seem to do so in the Pan et al. model. These differences may arise because different non-sensory cells were targeted: all cells were randomly targeted in our model, whereas Col2alCre activity determined the scope of the targeted cell population in the Pan et al. model.

The Hartman et al. model ${ }^{15}$ is similar to ours in that NICD is constitutively overactivated. The tamoxifen-independent $h G F A P C$ re bypassed the dystocia problem encountered in our model, thereby allowing the analysis of adult/juvenile inner ears. In 
the Hartman et al. model, presence of ectopic HCs at adult ages suggests that they can survive for a long time and are possibly functional. However, $h G F A P C r e$ will overactivate NICD soon after Cre is active and this model can therefore not be used to induce Notch1 signaling at various ages. Our tamoxifen-dependent $C A G^{\mathrm{CreER+}}$ allowed the overactivation of NICD at E10.5, E13 and P0/P1, in which we were able to show that there is an age-dependent decrease in the responsiveness of inner ear non-sensory cells to Notch1 signaling.

\subsubsection{Implication of Notch1 signaling in $\mathrm{HC}$ regeneration in mammals}

Similar to the chick inner ear study, ${ }^{20}$ our study and two other reports ${ }^{13,15}$ show that either constitutive or transient overactivation of NICD can induce ectopic HCs in mice. These results can have significant implications on studies of $\mathrm{HC}$ regeneration after $\mathrm{HC}$ damage in mammals. The non-sensory cells adjacent the endogenous sensory epithelium might be good candidates for manipulating Notch1 signaling.

Not all EGFP+ patches contain ectopic HCs and only embryonic non-sensory cells respond to NICD overactivation and generate new HCs, which highlights that other factors or signals besides Notch1 signaling may be needed to generate a bona fide sensory epithelium permissive for mechanosensory $\mathrm{HC}$ formation. Consistent with these data, Notch1 signaling has been shown to be required to maintain but not to initiate prosensory patch formation. ${ }^{113}$ Although these factors have yet to be identified, previous studies have shown that during cochlear morphogenesis, $\mathrm{Fgfr}^{114,115}$ and $\mathrm{Wnt}^{116,117}$ signaling pathways are involved in patterning the prosensory area. It is possible that a combined modulation of these signals triggers the conversion of postnatal non-sensory cells to HCs. 


\section{CHAPTER 5. ECTOPIC ATOH1 REPROGRAMS SUPPORTING CELLS INTO HAIR CELLS}

\subsection{Introduction}

Reprogramming one cell type into another after cell damage is an effective approach used in regenerative medicine. ${ }^{118}$ The landmark study where four key transcription factors can successfully reprogram somatic fibroblast cells into pluripotent embryonic stem cells highlights the concept that individual cell fate and function is mainly determined by combinations of different lineage specific transcription factors. ${ }^{119}$ This concept was further supported by a series of studies including reprogramming fibroblasts into neurons, ${ }^{120}$ cardiomyocytes ${ }^{121}$ and reprogramming pancreatic exocrine cells into their lineage-related insulin producing beta cells ${ }^{122}$ by overexpressing different specific linage transcription factors. Such lineage reprogramming therefore establishes a general basis for regenerative medicine including how to regenerate auditory hair cells (HCs) after HC damage to heal human deafness.

Atohl has been suggested to be a good candidate gene to define the HC fate, and possibly used for $\mathrm{HC}$ regeneration in mammals. In vitro overexpression of Atoh 1 in the non-sensory GER region of neonatal rat cochlear explants generates ectopic $\mathrm{HCs},{ }^{43}$ which supports the sufficiency of Atoh1 in specifying a HC fate in a permissive cellular environment. In vivo overactivation of Atoh1 in mouse otocysts ${ }^{41}$ and in adult cochleae of guinea pig $^{42}$ also leads to ectopic HCs.

Unfortunately, in these studies Atoh1 was overactivated in all cell types, making it impossible to determine, at single cell resolution, whether each individual ectopic HC is born from the endogenous $\mathrm{HC}$ lineages or from the SC lineage. Also, it remains elusive whether these ectopic HCs express prestin, a terminal differentiation marker responsible for the cochlear amplifier. ${ }^{123,124}$ Moreover, it is undetermined which SC subtype within the organ of Corti can give rise to inner or outer HCs. In addition, it is unclear whether constitutive or transient Atoh1 overexpression is required for in vivo reprogramming of SCs into HCs. Finally, it is unclear whether postmitotic mouse SCs at different postnatal ages still respond to ectopic Atoh1 expression and can be reprogrammed into HCs.

To induce ectopic Atoh1 expression at different developmental stages, we generated a CAG-flox-stop-flox-Atohl-HA+ (hereafter named as Atohl-HA+) transgenic mouse model. By crossing Atohl-HA+ mouse line with different SC specific CreER mouse lines (i.e. Plp ${ }^{\mathrm{CreER}+}$, Prox $1^{\mathrm{CreER+}}$ or $\mathrm{Fgfr} 3^{\mathrm{iCreER+}}$ ), ectopic Atoh1 expression is induced in SCs specifically at different postnatal ages, permitting single cell resolution analysis. 


\subsection{Materials and Methods}

\subsubsection{Mouse strains}

The Atohl-HA+ transgenic mouse line was generated with the following steps: 1) the Atoh1-HA construct was designed by creating primers that attached two HA tags to the 3' end of the pGEM-T-EASY Atoh1 from Dr. Martine Roussel's laboratory in St. Jude Children's Research Hospital; 2) It was then put back into the PGEM-T-EASY Vector; 3) Not I restriction enzyme was used to cut out the Atoh1-HA; 3) The vector pCAGGS-loxp-stop-loxp-IRES-GFP (from Dr. Tyler Jacks's laboratory in Massachusetts Institute of Technology) was also digested with Not I to remove the IRES-GFP; 4) the Atoh1-HA was then ligated into the pCAGGS-loxp-stop-loxp vector. The final size of the fragment after the backbone was digested out with Sal I was $4.8 \mathrm{~Kb}$.

$P l p^{C r e E R+}$ mice were purchased from Jackson Laboratory (USA). Prox $1^{\text {CreER/+ }}$ mice were kindly provided by Dr. Guillermo Oliver's laboratory in St. Jude Children's Research Hospital. Fgfr $3^{i C r e E R+}$ mice were kindly provided by Dr. William Richardson's laboratory in the University College London (UK). CAG-loxp-stop-loxp-EGFP+ reporter mice were kindly provided by Dr. Jeffrey Robbins's laboratory in the Cincinnati Children's Hospital Medical Center. Atoh1-EGFP+ mice were kindly provided by Dr. Jane Johnson's laboratory in the University of Texas Southwestern Medical Center.

\subsubsection{Antibodies and reagents}

With the same experimental procedures described in Chapter 2, the following antibodies were used: anti-Myosin-VI (rabbit, 1:200, 25-6791, Proteus Bioscience), anti-Calbindin (rabbit,1:500, AB1778, Millipore), anti-Parvalbumin (mouse, 1:2000, P3088, Sigma), anti-HA (rat, 1:100, 11867431001, Roche), anti-Prestin (goat, 1:200, sc-22692, Santa Cruz Biotechnology), anti-Lhx3 (rabbit, 1:2000, AB3202, Millipore), anti-GFP (chicken, 1:1000, ab13970, Abcam), anti-Sox2 (goat, 1:1000, sc-17320, Santa Cruz Biotechnology), anti-espin (a gift from Dr. Stefen Heller in Stanford University). The following secondary antibodies from Invitrogen Company were used: chicken anti-rat Alexa Fluor 488 (A-21470), goat anti-rat Alexa Fluor 568 (A11077), goat anti-rabbit Alexa Fluor 647 (A21245), donkey anti-rabbit Alexa Fluor 647 (A31573), donkey anti-goat Alexa Fluor 568 (A11057), goat anti-rabbit Alexa Fluor 568 (A11036), goat anti-mouse Alexa Fluor 647 (A21236) and goat anti-chicken Alexa Fluor 488 (A11039). All different secondary antibodies were used as 1:1000 dilutions. All images were taken with a confocal microscope (Zeiss LSM 700).

\subsubsection{Quantification of newly generated hair cells}

The cochlear samples are cut into three parts. After scanning each part with a confocal microscope at $10 \times$ lens, we measured the total length of the cochleae. Then, 
each cochlea was divided into three turns (basal, middle and apical) with equal length. For the Prox $1^{\mathrm{CreER/+}}$; Atohl-HA+ model, because of the limited number of new HCs, each entire cochlea was Z-stack scanned with $40 \times$ lens $\left(1 \mu \mathrm{m}\right.$ interval). For $\mathrm{Fgfr}^{\mathrm{iCreER+}}{ }^{+}$; Atoh $1-H A+$ model, because there were many new HCs with relatively even distribution across the cochlear turns, two regions at each turn were randomly chosen and an averaged $\mathrm{HC}$ number was calculated in each sample. For the $\mathrm{Plp}^{\mathrm{CreER+}}$; Atoh $1-\mathrm{HA}+$ model, the new HCs across the entire cochlear turns were quantified at P6 when only a few new HCs were present. Two regions at each turn were randomly chosen and an averaged $\mathrm{HC}$ number was calculated in each sample at P22 or P60 when many new HCs were present and their distribution was even.

\subsection{Results}

\subsubsection{Generation of inducible Atoh1 overexpressing mouse lines}

Atoh1 is normally undetectable in SCs at postnatal ages. ${ }^{39,40}$ Here, we focused on three subtypes of SCs, inner phalangeal cells (IPHs), Pillar cells (PCs) and Deiters' cells (DCs) that are directly underneath the endogenous HCs, with the belief that they are good sources to regenerate HCs in situ (Figure 5-1A). To reactivate Atoh1 in postnatal IPHs, PCs and DCs, we generated inducible Atohl-HA+ transgenic mouse lines in which Atoh1 (tagged with HA at the C-terminus) is driven by the $C A G$ promoter but its expression is blocked until the "STOP" fragment is deleted in a Cre-mediated manner (Figure 5-1B). In addition, HA tag does not affect the activity of Atoh1, as wild type cerebellum granule neuronal progenitors (GNPs) transfected with retroviruses expressing the same HA-tagged Atoh1 and Gli1 led to medulloblastoma twice as rapidly as GNPs transfected with retroviruses expressing Gli1 alone (unpublished observation from Dr. Martine Roussel's laboratory), when they are orthotopically transplanted into the cortex of CD1-nu/nu recipient mice. ${ }^{125}$

We obtained four different transgenic founders after pronuclear injection of the transgenic construct. While all four founders (10,11, 12 and 19) expressed ectopic Atoh1-HA expression in IPHs, PCs and DCs when crossed with different CreER mouse lines, lineage reprogramming of IPHs, PCs and DCs into HCs only occurred in founder 10. Therefore we focused on the founder 10 for subsequent analyses.

\subsubsection{Neonatal inner phalangeal cells (IPHs) can be reprogrammed into inner hair cell-like cells}

Analysis of $P l p^{\mathrm{CreER}^{+}}$; Rosa26-Lac ${ }^{\text {loxp/+ }}$ mice showed that Cre activity is limited to IPHs inside the organ of Corti. ${ }^{126}$ We also independently analyzed $P l p^{C r e E R+}$; Rosa26-EYFP ${ }^{l o x p /+}$ mice at $\mathrm{P} 6$ which were subjected to tamoxifen at $\mathrm{P} 0$ and $\mathrm{P} 1$. Inside the organ of Corti, most of the EYFP traced cells were IPHs, while a small fraction were PCs and DCs. EYFP+ HCs were never observed. Because there is no difference among 

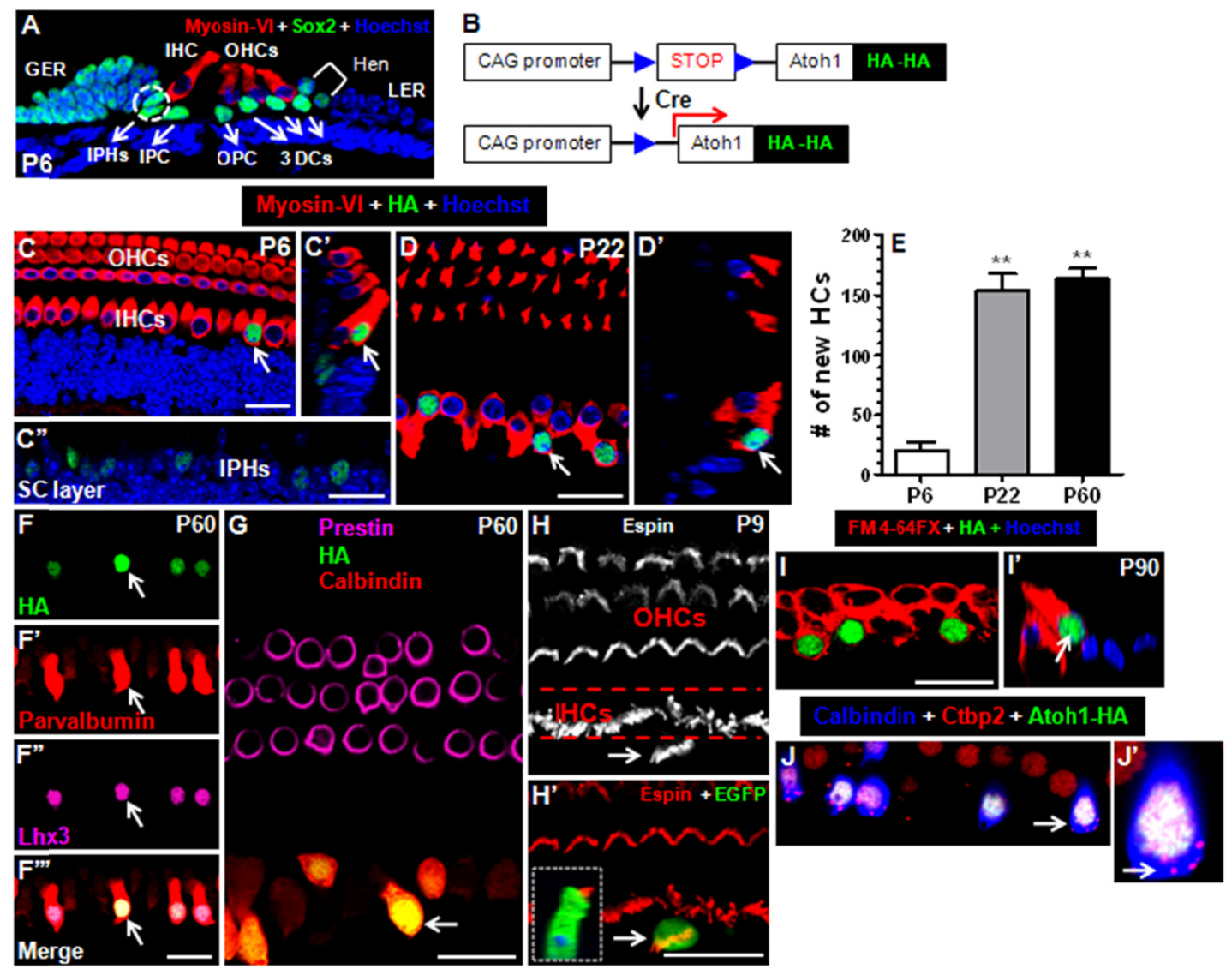

Figure 5-1 Atoh1 reprogrammed neonatal inner phalangeal cells (IPHs) to hair cells.

(A) Histology of the mouse cochlea. (B) Scheme of ectopic Atoh1-HA expression. (C-D') Double staining of Atoh1-HA and Myosin-VI of cochlear samples from $\mathrm{Plp}^{\mathrm{CreER+}}$; Atoh $1-H A+$ mice that were given tamoxifen at $\mathrm{P} 0$ and $\mathrm{P} 1$, and analyzed at $\mathrm{P} 6$ (C-C' ') and P22 (D-D'). Arrows point to the same new HCs. (E) Quantification of the new HCs at P6, P22 and P60 (** p<0.01). (F-F',') Triple staining of Atoh1-HA, Parvalbumin and Lhx3. Arrows label the same new HCs. (G) Triple staining of Atoh1-HA, Calbindin and Prestin. Arrow labels the new HC. (H-H') EGFP and espin double staining of cochlear samples from $\mathrm{Plp}^{\mathrm{CreER+}}$; Atohl-HA+; CAG-EGFP+ mice. Arrows label the same new HC that were traced with EGFP (originated from IPHs) and were espin+. (I-I') FM-4-64 dye and Atoh1-HA double staining visualized at confocal XY plane (I) or YZ plane (I'). The arrow labels one new HC that could uptake the red FM-4-64 dye. (J-J') Double staining of Calbindin, Ctbp2 and Atoh1-HA visualized in XY plane (J) or YZ plane (J'). Arrows label the same new HC that had punctuated Ctbp2 staining. Bars: $20 \mu \mathrm{m}$. 
different cochlear turns, we grouped three turns together and normalized EYFP+ cells to the total number of each SC subtype. About $47.71 \%$ of IPHs were EYFP+, and $3.42 \%$ of PCs and $5.16 \%$ of DCs were EYFP+ as well (Figure 5-2). Because the majority of EYFP+ cells were IPHs, we used $P l p^{C r e E R+}$ mice to determine whether ectopic Atoh1 can reprogram IPHs into HCs.

We crossed Plp ${ }^{\mathrm{CreER}+}$ with Atohl-HA+ mice to obtain $\mathrm{Plp}^{\mathrm{CreER+}}$; Atohl-HA+ mice as the experimental group, while Plp ${ }^{\mathrm{CreER}-}$; Atoh1-HA+ or Plp $\mathrm{CreER+}^{\text {; Atohl-HA- }}$ littermates were the control group. Both experimental and control groups received identical treatments: first with tamoxifen at $\mathrm{P} 0$ and $\mathrm{P} 1$, and second analysis at various ages. In our study, we used the HA antibody to label and visualize ectopic Atoh1 expression. Permanent Atoh1-HA expression allowed us to perform lineage tracing of the Atoh1-HA+ PCs and DCs, and to further determine whether they could be reprogrammed into HCs by co-labeling Atoh1-HA and different HC specific markers. Many Atoh1-HA+ cells $(830 \pm 40, n=3)$ were observed across the entire cochlear turns at P6, among which $2.5 \%$ of Atoh $1-\mathrm{HA}+$ cells $(20 \pm 6, n=3)$ migrated up to $\mathrm{HC}$ layer and turned on Myosin-VI (Figure 5-1C-C'), which is the earliest marker to define a HC fate within the organ of Corti. ${ }^{127,}{ }^{128}$ Intriguingly, the majority of the Atoh1-HA+/Myosin-VI- IPHs remained in the SC layer (Figure 5-1C').

When samples were analyzed at P22, however, there were much more Atoh1-HA+/Myosin-VI+ cells $(153 \pm 14, n=3)$ across the entire cochlea, occupying about $18 \%$ of the total Atoh1-HA+ cells $(851 \pm 36, n=3)$ (Figure 5-1D-D'). The number of Atoh1-HA+/Myosin-VI+ cells was not further increased when samples were analyzed at P60 (Figure 5-1E). In addition, Atoh1-HA+/ Parvalbumin+/Lhx3+ (Figure 5-1F-F'"') or Atoh1-HA+/Calbindin+/Prestin-negative cells (Figure 5-1G) were present across the entire cochlear turns. Calbindin and Parvalbumin are normally expressed in HCs but not SCs. ${ }^{129} \mathrm{Lhx} 3$ is a transcriptional factor that is expressed during the $\mathrm{HC}$ differentiation process and genetic interactions among Lhx3, Pou $4 \mathrm{f} 3$ and Atoh1 have been proposed previously. ${ }^{130,131}$ It suggests that with ectopic Atoh1 expression some but not all IPHs can be reprogrammed into $\mathrm{HC}$ fate and turn on multiple $\mathrm{HC}$ specific markers, and that the reprogramming speeds are heterogeneous among different IPHs. Cells deriving from $\mathrm{Cre}+\mathrm{IPHs}$ and reactivated $\mathrm{HC}$ markers were tentatively defined as new HCs. Interestingly, the Atoh1-HA expression level in these HCs was always much higher than that in Atoh1-HA+ IPHs which did not reactivate HC specific markers (or were not successfully reprogrammed). While $43 \%$ of the new HCs lost Sox 2 expression, $57 \%$ of them still maintained Sox2, suggesting a potential different extent of the reprogramming among HC-like cells. Lastly, in either of the control groups, we never saw HA+ cells nor ectopic HCs, confirming that our Atoh1-HA+ transgene was not expressed in the absence of Cre-mediated recombination.

The cilia structure on the top surface, referred as to stereocilia, is one of the characteristics of HCs. OHCs have the "V" or "W" shaped stereocilia and IHCs have the straight line shaped stereocilia, which makes it possible to further determine whether the new HCs are OHCs or IHCs. We crossed CAG-flox-stop-flox-EGFP+ (hereafter $C A G-E G F P+)$ mice $^{132}$ with $P l p^{\text {CreER+ }}$; Atohl-HA+ mice. 

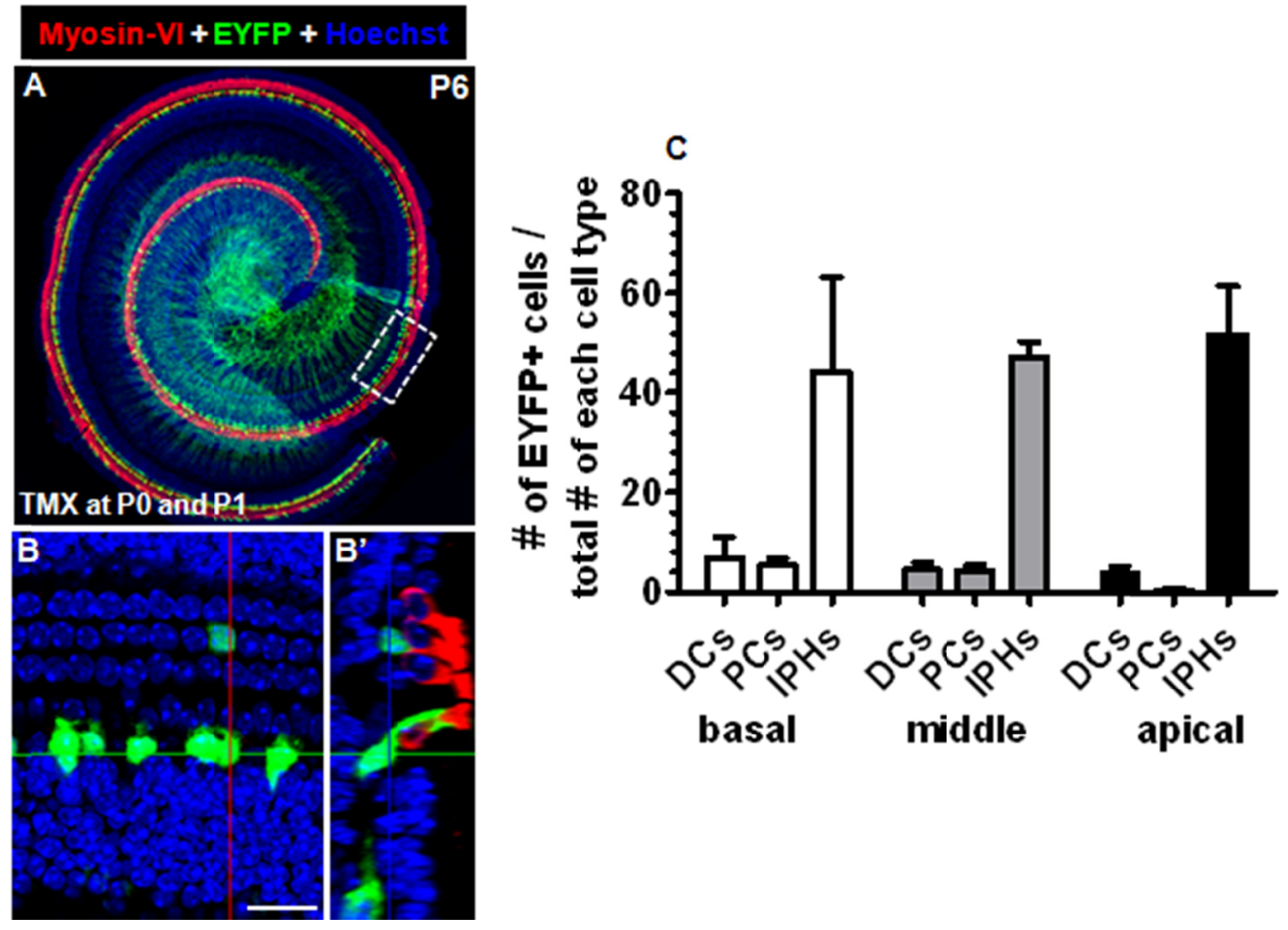

Figure 5-2 Characterization of Cre activity in $\mathrm{Plp}^{\mathrm{CreER+}}$ mouse.

(A) $P l p^{\mathrm{CreER}^{+}} ; \mathrm{R} 26-\mathrm{EYFP}^{\mathrm{Loxp} /+}$ mice were injected with tamoxifen at $\mathrm{P} 0$ and $\mathrm{P} 1$, and analyzed at P6. Image was taken in the confocal XY plane. (B-B') High magnification image of the white rectangular region in (A). Image (B) was taken in the confocal XY plane, and image (B') was in the confocal YZ plane. (C) Percentage of the EYFP+ cells among each SC subtypes. The majority of EYFP+ cells were IPHs. Bars: $20 \mu \mathrm{m}$. 
Plp ${ }^{\mathrm{CreER+}}$; Atoh1-HA+; CAG-EGFP+ mice were treated with tamoxifen at $\mathrm{P} 0$ and $\mathrm{P} 1$ and analyzed at $\mathrm{P} 9$ (Figure 5-1H-H'). The actin-binding protein espin was used to visualize stereocilia. ${ }^{133}$ Expression of espin on the top surface of the EGFP+ cells clearly showed that the stereocilia was formed at P9 in these newly generated HCs (EGFP+, derived from Cre+ IPHs). Furthermore, the stereocilia morphology of the new HCs is very similar to that of the endogenous inner but not outer HCs. Consistently, these HC-like cells did not express prestin, ${ }^{123}$ an OHC specific marker (Figure 5-1G). It supports that IPHsderived HCs adopt the inner hair cell fate.

Presence of stereocilia in the new HCs prompted us to further determine whether they had the electrophysiological function of wild type HCs. The initial step of sensing sound starts at the opening of the mechanosensory transduction (MET) channel located in the stereocilia. Whole mount prepared cochlear epithelium of $\mathrm{Plp}^{\mathrm{CreER+}}$; Atoh $1-\mathrm{HA}+$ or Plp ${ }^{\mathrm{CreER}^{+}}$; Atohl-HA+; CAG-EGFP+ were exposed transiently (30 second incubation only) to the FM4-64FX dye at P13, P30 and P90. We found that the FM4-64FX dye was taken up into Atoh1-HA+ or EGFP+ new HCs at all ages (Figure 5-1I-I'). Furthermore, when cochlear samples were treated with FM4-64FX dye and reagents interfering or blocking the MET channel, the intensity of FM4-64FX inside the Atoh1-HA+ or EGFP+ new HCs were either absent or dramatically decreased (Figure 5-1I-I'). Taken together, it suggests that the MET channel is present and presumably functional in the new HCs.

To determine whether these new HCs were innervated by spiral ganglion neuronal fibers, CtBP-2 was used to visualize the presynaptic ribbons. ${ }^{134}$ Presence of CtBP-2+/Atoh1-HA+/Calbindin+ cells strongly suggests that synapses are formed between HC-like cells and spiral ganglion neurons (Figure 5-1J-J'), which was further supported by the fact that Tuj1+ neuronal fibers were connected to these HC-like cells. Moreover, the distribution of CtBP-2 in the synaptic regions of these new HCs appeared punctate, similar to what was reported in inner HCs, ${ }^{134}$ again supporting that these IPH-derived HCs are functional inner HCs. Further conclusive experiments are needed to prove the functionality of IPHs-derived new HCs.

Last, because only very limited IPHs could be targeted when tamoxifen was injected at P12 and older ages, we did not analyze the response of the juvenile IPHs to ectopic Atoh1-HA expression.

\subsubsection{Ectopic Atoh1-HA reprogrammed neonatal pillar and Deiters' cells to hair cells}

To induce ectopic expression of Atoh1-HA specifically in neonatal PCs and DCs, we crossed Proxl ${ }^{\mathrm{CreER} /+}$ mice with Atohl-HA+ mice. The Cre activity of Proxl ${ }^{\mathrm{CreER} /+}$ was limited to PCs and DCs when tamoxifen was given at $\mathrm{P} 0$ and $\mathrm{P} 1 .{ }^{73}$ Prox $^{\mathrm{CreER} /{ }^{+}}$; Atoh1-HA+ mice were experimental groups, while Prox ${ }^{\text {CreER/+ }}$; Atoh1-HA- or Prox $1^{+/+}$; Atohl-HA+ mice were used as control groups. All experimental and control mice from the same litters received the same tamoxifen treatment at P0 and P1. Similar to Plp ${ }^{\mathrm{CreER+}}$; Atohl-HA+ mouse models, we used HA antibody to label and visualize ectopic 
Atoh1-HA expression. Consistent with the graded Cre activity of Prox $1^{\text {CreER/+ }}$ in different turns, ${ }^{73} \sim 5 \%$ of PCs and $\sim 16 \%$ of DCs were Atoh1-HA+ in apical turns, while $\sim 3 \%$ of PCs and $\sim 7 \%$ of DCs were Atoh1-HA+ in middle and basal turns of Prox $1^{\text {CreER+ }}$; Atoh1-HA+ mice at P6. Again, permanent Atoh1-HA expression allowed us to perform lineage tracing of the Atoh1-HA+ PCs and DCs, and to further determine whether they could be reprogrammed into HCs by co-labeling Atoh1-HA and different $\mathrm{HC}$ specific markers.

We analyzed samples at P6, P12, P22, P60 and P90. P22 is the earliest age when we could find Atoh1-HA+/Myosin-VI+, Atoh1-HA+/Calbindin+ and Atoh1-HA+/Parvalbumin+ cells (Figure 5-3A-C). These cells expressing Atoh1-HA (derived from PCs or DCs) and multiple HC markers were defined as new HCs at single cell resolution. The number of new HCs per cochlea was much higher at $\mathrm{P} 60(30 \pm 6$, $n=3)$ than P22 (12 $\pm 2, n=3)$ (Figure 5-3D). The number of new HCs was not further increased at P90. This supports the idea that different PCs and DCs will take different times to be reprogrammed. Similar to the IPHs derived new HCs, Atoh1-HA expression level in the new HCs always seemed much higher than the Atoh1-HA+ PCs and DCs that did not reactivate multiple HC markers (Figure 5-3E-E'). These new HCs migrated to the $\mathrm{HC}$ layer and resided in $\mathrm{OHC}$ regions, causing cell death of endogenous OHCs. Among the total Atoh1-HA+ PCs and DCs at P60 or P90, 6\% $\pm 2 \%(n=3)$ can be reprogrammed into HCs.

To determine whether the new-HCs could be fully differentiated, we further performed double labeling of Atoh1-HA and prestin, which is a terminal differentiation marker for OHCs and required for the cochlear amplifier. ${ }^{123,124}$ All the new HCs did not express prestin at P22 (Figure 5-3A-A') or P90 (data not shown), suggesting they are not fully differentiated. To confirm our data, we further analyzed the Prox $1^{\mathrm{CreER} /+}$; Atoh1-HA+; CAG-EGFP+ mice at P30 $(n=3)$ or $\mathrm{P} 60(n=3)$. Espin is an actin binding protein highly expressed in HC stereocilia. Absence of the EGFP+/Espin+ cells suggests that there were no obvious stereocilia in the surface of the new HCs (Figure 5-3F). In addition, their overall round morphology suggests that they are not properly polarized (Figure 5-3). Last, in neither of the control groups, did we observe Atoh1-HA expression, ectopic HCs or sporadic endogenous OHC loss.

\subsubsection{Juvenile pillar and Deiters' cells with ectopic Atoh1-HA also can be reprogrammed to hair cells}

Because human deafness can occur at any postnatal ages due to various ototoxic factors, it is of particular interest to determine whether juvenile PCs and DCs (at P6 and P7 or P12 and P13) that are in the process of differentiation can still respond to ectopic Atoh1-HA expression and be reprogrammed to HCs. As Prox1 declines rapidly after birth in mouse SCs, ${ }^{83}$ Prox $1^{\mathrm{CreER} /+}$ cannot be used to drive ectopic Atoh1-HA expression. We therefore used the $\mathrm{Fgfr} 3^{\mathrm{iCreER+}}$ mouse line. We analyzed Fgfr $3^{i C r e E R+}$; Atohl-HA+ mice, in comparison with Fgfr $3^{\text {iCreER+ }}$; Atohl-HA - or Fgfr $3^{\text {iCreER- }}$; Atohl-HA+ littermate controls. We analyzed $\mathrm{Fg} f r 3^{\text {iCreER+ }}$; Atoh1-HA+ mice one month (P36) after tamoxifen 

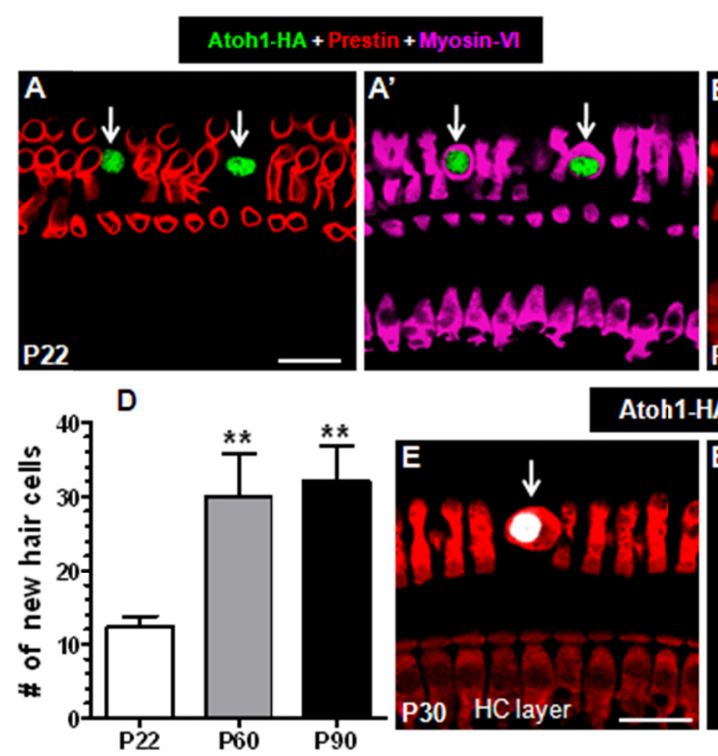
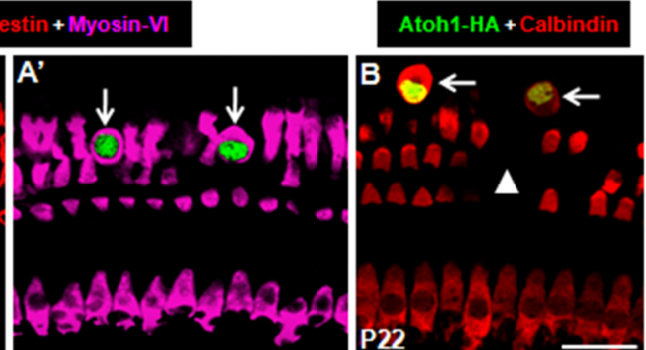

HA + Calbindin

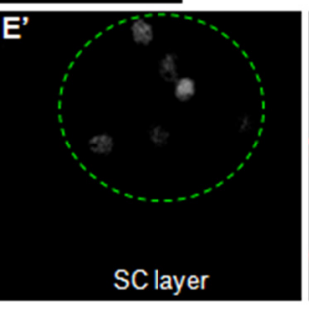

Atoh1-HA + Parvalbumin

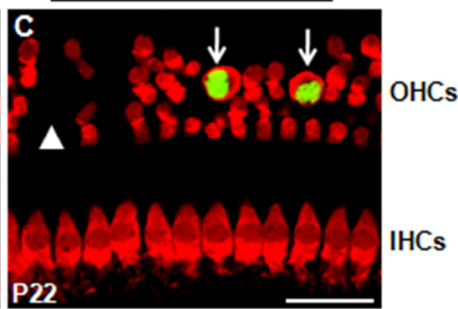

EGFP + Espin

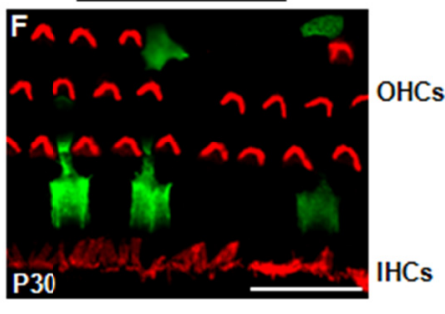

Figure 5-3 Reprogramming of neonatal PCs and DCs into HCs by ectopic expression of Atoh1-HA.

(A-A') Triple staining of HA, prestin and Myosin-VI. Arrows are the two newly generated HCs. (B-C) Double labeling of HA with Calbindin (B) or Parvalbumin (C). Arrows are the generated HCs. Arrows heads point to the regions where endogenous OHCs are missing. (D) Quantification of the new HCs at P22, P60 and P90 (** p<0.01). (E-E') Comparison of Atoh1-HA expression level between new HCs (the arrow in E) and Atoh1-HA+/Calbindin-negative PCs or DCs (inside the green dotted circle in E'). (F) Double labeling of EGFP and espin in cochlear samples from Prox $1^{\mathrm{CreER} /+}$; Atoh 1-HA+; $C A G-E G F P+$ mice. No EGFP+/espin+ cells were found. Bars: $20 \mu \mathrm{m}$. 
injection P6 and P7, or P42 when tamoxifen were given at P12 and P13. Because of the similar phenotypes between the two tamoxifen treatment schedules, here we only described in details the latter one. About $80 \% \pm 5 \%(n=3)$ of the PCs and DCs were Atoh1-HA+. In the averaged $160 \mu \mathrm{m}$ cochlear length, $11 \pm 2(\mathrm{n}=4)$

Atoh1-HA+/Calbindin+ cells (defined as new HCs) were found in the $\mathrm{OHC}$ region at P42, which occupied approximately $11.7 \%$ of the total Atoh1-HA+ PCs and DCs (Figure 5-4A-A" '). But none of the new HCs expressed prestin (arrows in Figure 5-4A-A"'). Additionally, these new HCs also expressed Parvalbumin and Lhx3 (Figure 5-4B-B','). The juvenile PCs and DCs derived new HCs migrated to the HC layer, resulting in cell death of endogenous OHCs. As expected, in either control group, there were no endogenous $\mathrm{OHC}$ loss and no newly generated HCs.

Studies in other tissue cells support the notion that endogenous master cell fate determination genes need to be turned on for efficient reprogramming. ${ }^{122,135,136}$ To determine whether in the new HCs the external Atoh1-HA could turn on the endogenous Atohl at single cell resolution, $\mathrm{Fgfr} \mathrm{CreER+}^{\mathrm{i}}$; Atoh $1-\mathrm{HA}+$ mice were bred with the transgenic Atohl-EGFP+ mice where EGFP is driven by Atoh1 enhancer. ${ }^{137}$ In Fgfr $3^{\text {iCreER+ }}$; Atoh1-HA+; Atohl-EGFP+ mice that were treated with tamoxifen at P12 and P13, EGFP was used to reflect the activation of endogenous Atohl due to the positive auto-regulation of Atoh $1 .{ }^{138}$ In the averaged $160 \mu \mathrm{m}$ cochlear length, $10 \pm 4$ $(n=3) \mathrm{EGFP}+/$ Atoh1-HA+/Calbindin+ cells were found at P42 (Figure 5-5A-A"'), Interestingly, Atoh1-HA+/Calbindin- PCs and DCs were EGFP-(Figure 5-5B-C','). The EGFP expression in endogenous HCs was faint that could only by detectable when the confocal laser had to be switched to a high power, in which condition EGFP signals in the Atoh1-HA+/Calbindin+/EGFP+ cells had been already oversaturated.

\subsubsection{Adult pillar and Deiters' cells are insensitive to ectopic Atoh1-HA expression}

We determined whether ectopic Atoh1 reprogrammed adult fully differentiated PCs and DCs into HCs. When $\mathrm{Fgfr}^{\mathrm{iCreER+}}$; Atoh $1-\mathrm{HA}+$ mice were treated with tamoxifen once at P30, 90\% of PCs and $\sim 50 \%$ of DCs were Atoh1-HA+ at P60. Surprisingly, we no longer observed $\mathrm{HA}+/$ Calbindin+ newly generated HCs or OHC loss (Figure 5-6A-C). We further analyzed mice at P90 and P120 and allowed Atoh1-HA+ PCs and DCs to have longer time to respond to Atoh1-HA expression (data not shown). Again, no new HCs were found. Consistently, 100\% of Atoh1-HA+ PCs and DCs maintained expression of SC marker Sox2 (Figure 5-6D-D'”). Therefore, we concluded that fully matured PCs and DCs at P30 were not sensitive to ectopic Atoh1-HA expression.

Next we speculated that combination of prior OHC damage might promote adult PCs and DCs to respond to ectopic Atoh1-HA expression (Figure 5-6E). First, adult OHCs were damaged at P30 with Kanamycin and Furosemide. ${ }^{51}$ Second, OHC damage was further confirmed by auditory brainstem response (ABR) tests at P33, followed by tamoxifen injection once at P33. While there were many Atoh1-HA+ PCs and DCs, we did not find any new HCs and hearing impairment by drug damage was not improved 

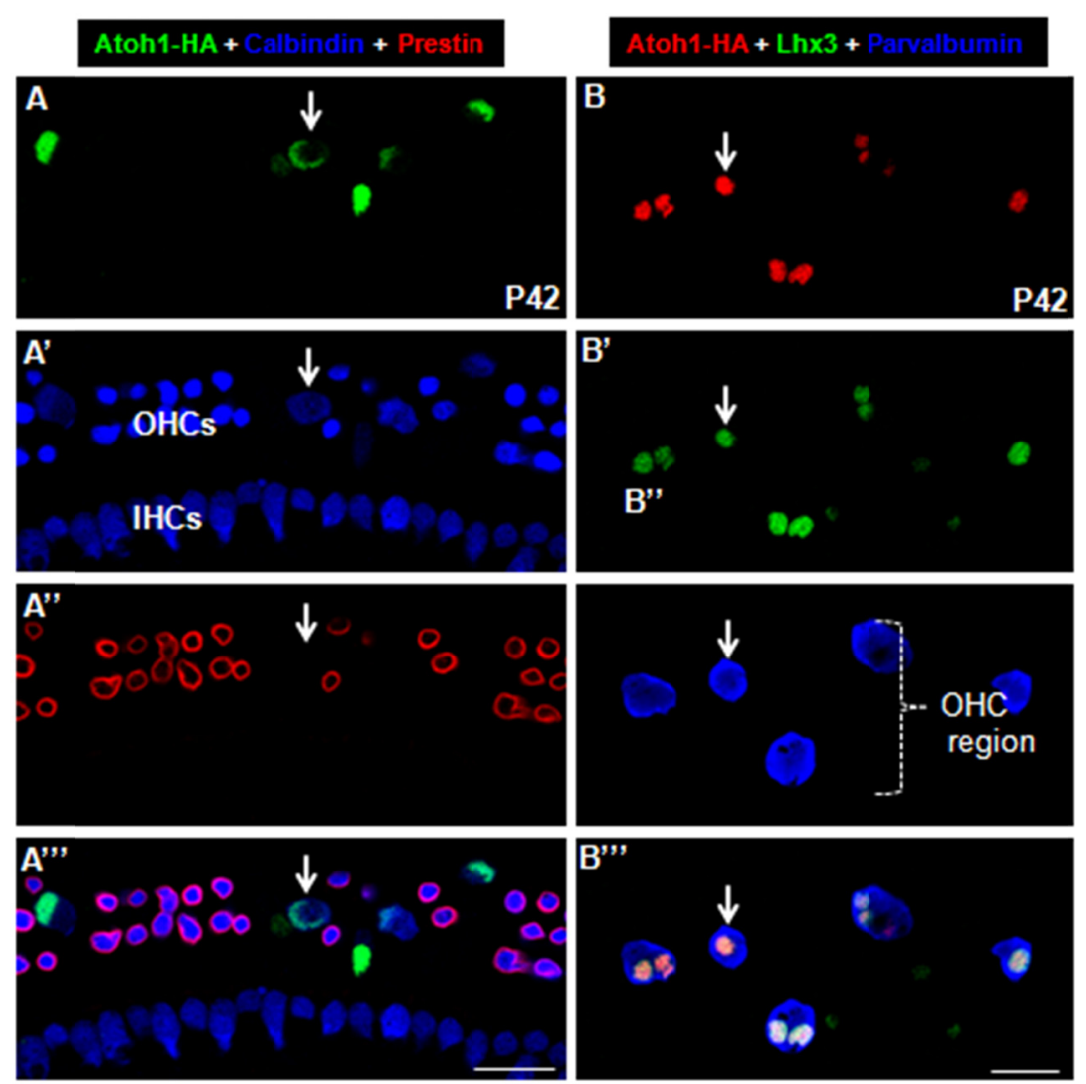

Figure 5-4 Ectopic Atoh1-HA reprogrammed juvenile PCs and DCs into HCs. (A-A"') Triple staining of HA, Prestin and Calbindin. Arrow is one new HC. Many endogenous prestin+ OHCs were missing, while IHCs were normal. (B-B"') Triple staining of HA, Parvalbumin and Lhx3. Arrow is one new HC. Arrow heads in (B') were endogenous Lhx3+ HCs. Note that the endogenous HCs actually had Parvalbumin but it was hard to visualize with the current confocal laser setup because the level was much lower than that in new HCs. OHCs: outer hair cells; IHCs: inner hair cells. Bars: $20 \mu \mathrm{m}$. 


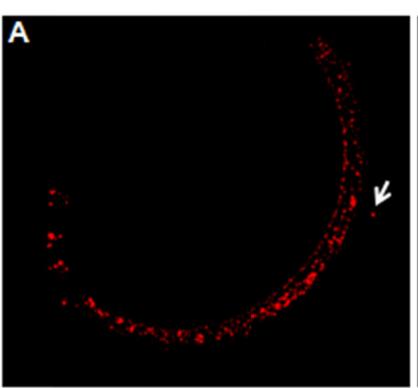

\section{HA + Calbindin + EGFP +}
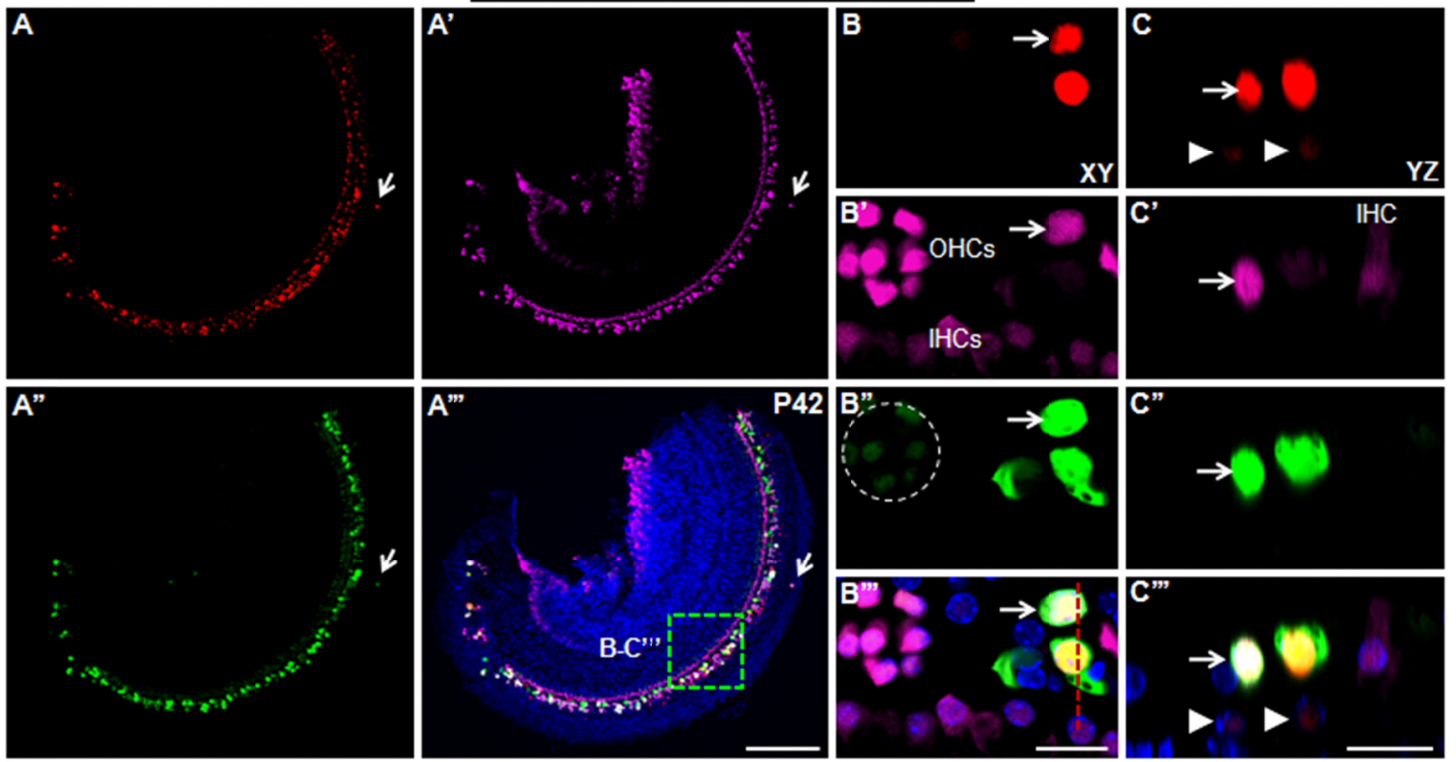

Figure 5-5 Endogenous Atoh1 gene in new HCs is reactivated.

(A-A"') Triple labeling of Atoh1-HA, Calbindin and EGFP. Arrows label the new HC distributed in LER region. (B-C"') are the high magnification images of the square area in (A"'). Arrows are the same new HC visualized at confocal XY plane (B-B"') and YZ plane (C-C" "). Cells inside white dotted circle are the endogenous OHCs with faint EGFP expression. Two arrow heads in (C) are the Atoh1-HA+/Calbindin-negative PCs or DCs. Red vertical lines in (B"') is the angle to generate images in (C-C"'). OHCs: outer hair cells; IHCs: inner hair cells. Bars: $200 \mu \mathrm{m}$ (A"'); $20 \mu \mathrm{m}$ (B"', C'"). 

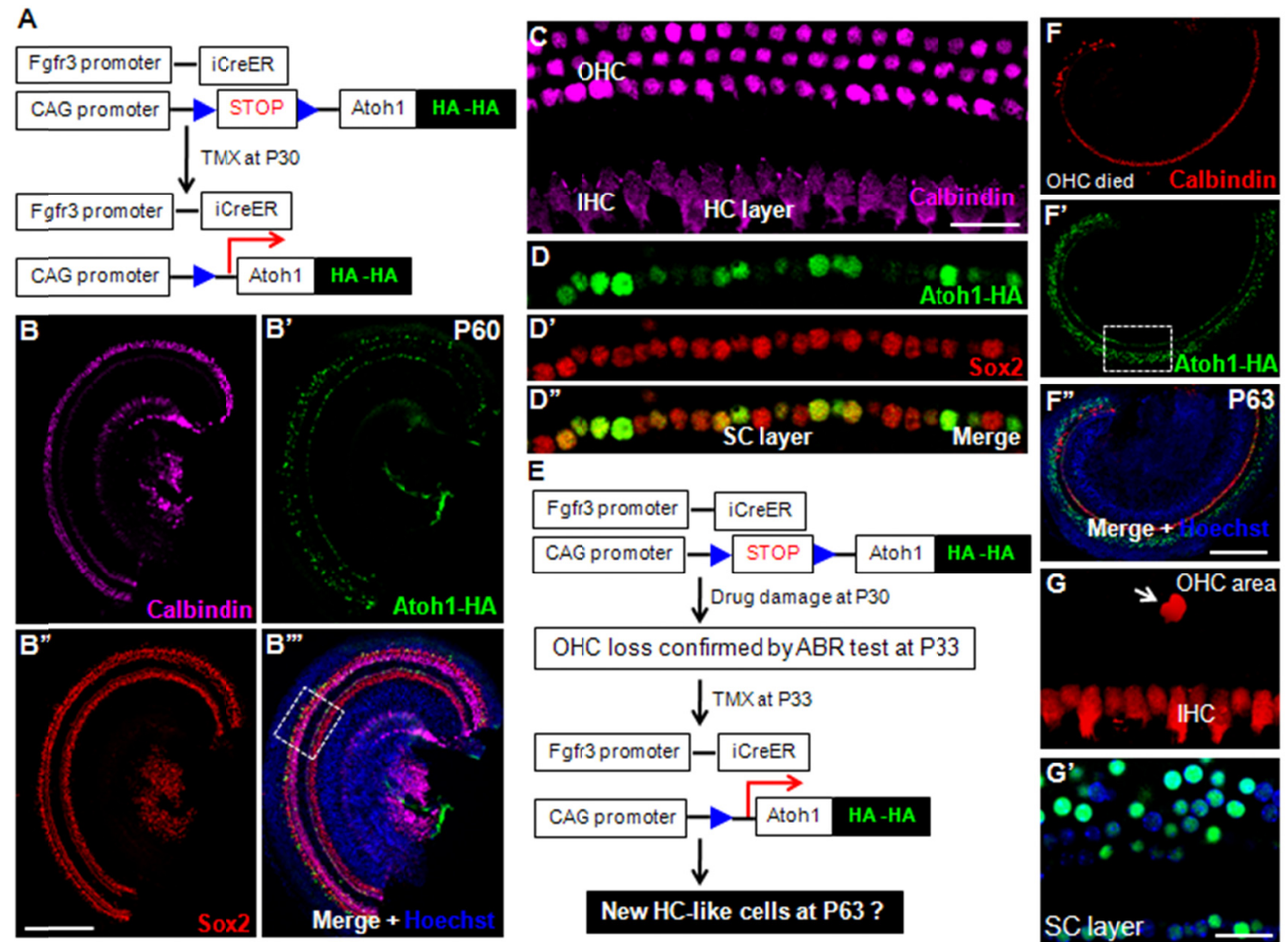

New $\mathrm{HC}$-ike celis at P63?

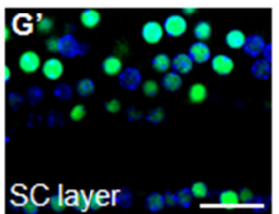

Figure 5-6 Adult PCs and DCs were insensitive to the ectopic Atoh1-HA.

(A) Illustration of the experimental schedule. (B-B'"') Low magnification images of triple staining of Atoh1-HA, Calbindin and Sox2. (C-D') are the high magnification images of the square area in (B','). Neither OHC loss nor new HCs were found at HC layer (C). Using inner pillar cells as example, all Atoh1-HA+ cells in (D-D' ') expressed Sox2. (E) Illustration of the experimental schedule for drug damage followed by turning on ectopic Atoh1. (F-F') Double labeling of Atoh1-HA and Calbindin. Almost all OHCs were damaged. (G-G') are the high magnification images of the square area in (F'). Arrow in $(\mathrm{G})$ is the single left OHC. All Atoh1-HA+ PCs and DCs in (G') did not turn on Calbindin. OHC: outer hair cell; IHC: inner hair cell. Bars: $200 \mu \mathrm{m}\left(\mathrm{B}^{\prime}\right.$, , F' '); $20 \mu \mathrm{m}(\mathrm{C}$, G'). 
when mice were analyzed one month later (P63) (Figure 5-6E-G') or two month later (P83). Alternatively, to allow adult PCs and DCs to have ectopic Atoh1-HA before OHC damage, we also tried tamoxifen injection at P30, and performed drug damage three days later (P33). Again, neither new HCs were found nor hearing improvement was achieved.

\subsubsection{Permanent expression of Atoh1-HA in differentiating endogenous hair cells causes cell death}

In the above models, ectopic Atoh1-HA expression is permanent. We found that PCs- and DCs-derived HCs may not be fully differentiated, even though multiple HC-specific genes were activated. This may be caused by the lack of additional unknown factors that are needed to fully reprogram PCs and DCs into mature HCs or that permanent Atohl expression prevents new HCs from completing terminal differentiation. While the former scenario remains an attractive idea to be explored in the future, we tested the latter by using the $\mathrm{Gfil}^{\mathrm{Cre} /+}$; Atohl-HA+ mouse model, with the assumption that embryonic differentiating endogenous HCs (when Gfil is turned on) are similar to $\mathrm{PC}$ and DC-derived new HCs. Inside the organ of Corti, Cre activity in $\mathrm{Gfil}^{\mathrm{Cre} / \mathrm{t}}$ mice starts at embryonic day (E) 15.5 and is specific to HCs; ${ }^{139}$ thus, in $\mathrm{Gfil}^{\mathrm{Crel+}}$; Atohl-HA+ mice differentiating endogenous HCs around E16 or E16.5 started to have both endogenous Atoh1 and ectopic Atoh1-HA expression in a basal to apical gradient.

We analyzed cochlear samples of $\mathrm{Gfil}^{\mathrm{Cre} /+}$; Atohl-HA+ mice at P3, P14 and P35. All HCs were Atoh1-HA+/Myosin-VI+ and looked normal at P3 ( $n=3)$ (Figure 5-7A-A" "); however, at P14, $60 \%$ of HCs were lost in the basal turn $(n=3)$, while middle and apical $\mathrm{HCs}$ were normal except for the most apical region where varied $\mathrm{OHC}$ loss was present (Figure 5-7B-E"'). The remaining IHCs were Atoh1-HA+/Calbindin+ and OHCs were Atoh1-HA+/Calbindin+/prestin+. When cochlear samples were analyzed at P35, $98 \%$ of $\mathrm{HCs}$ in the middle or basal turns, and $\sim 85 \%$ of $\mathrm{HCs}$ at the apical turn were lost (Figure 5-7F). Consistently, Gfi $1^{\mathrm{Cre} /+}$; Atohl-HA+ mice were completely deaf at $\mathrm{P} 35$ by ABR test (Figure 5-7G). This finding suggests that permanent Atoh1-HA expression is not the main cause for absence of prestin expression in the newly generated $\mathrm{HCs}$, that permanent Atohl-HA expression is not ideal for $\mathrm{HC}$ regeneration and that mature, but not immature HCs are vulnerable to ectopic Atoh1-HA expression. However, it is also possible that new HCs may have their unique differentiation program.

\subsection{Summary and Discussion}

We have achieved inducible ectopic Atoh1-HA expression specifically in three subtypes of postnatal cochlear SCs, IPHs, PCs and DCs, that reside directly underneath HCs within the organ of Corti. A fraction of neonatal or juvenile Atoh1-HA+ SCs were reprogrammed into HCs expressing HC markers (Myosin-VI, Calbindin, Parvalbumin, and Lhx3) and further survived for more than 3 months in vivo. The IPH-derived new $\mathrm{HCs}$ resembled the endogenous IHCs in many aspects. However, the newly generated HCs lacked Prestin expression and morphology of mature HCs, and remained at 


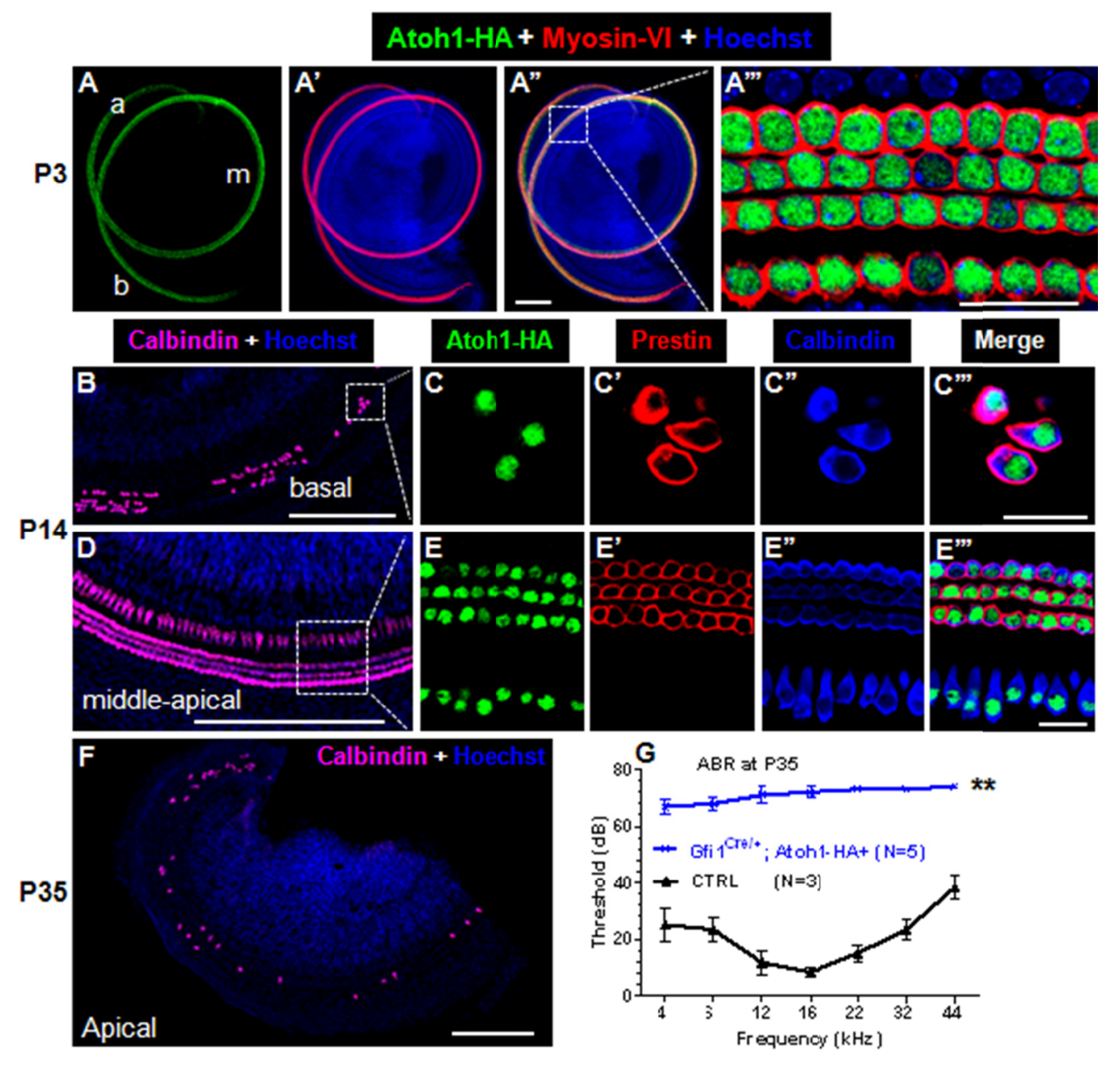

Figure 5-7 Effects of permanent Atoh1 expression in endogenous differentiating HCs.

(A-A,', Double labeling of Atoh1-HA and Myosin-VI of cochlear samples from Gfil $\mathrm{Cre}^{+}$; Atoh1-HA+ mice at P3. All Atoh1-HA+ HCs are normal. (B-E',') Triple staining of Atoh1-HA, Calbindin and prestin at P14 cochlear samples. Only Calbindin channel signal is shown in (B) and (D). Calbindin in (C') and (E') is shown as pseudo blue color. (F) Calbindin staining at P35 cochlear samples. (G) Auditory Brainstem Response (ABR) hearing comparison $\mathrm{Gfil}^{\mathrm{Cre} /+}$; Atohl-HA+ and $\mathrm{Gfil}^{\mathrm{Cr} /+}$ control mice. ${ }^{* *} \mathrm{p}<0.01$ in all frequencies. 
intermediate differentiation stages without full differentiation into functional mature $\mathrm{HCs}$ (especially the OHCs). However, the adult PCs and DCs were insensitive to ectopic Atoh1-HA expression. We also tested permanent Atoh1 expression in endogenous embryonic HCs which resulted in death of only mature, but not in the immature HCs. Taken together, we propose that either transient Atoh1 expression or other factors in addition to Atoh1 are required to reprogram PCs and DCs (especially at adult ages) into fully differentiated functional HCs.

\subsubsection{Identifying the newly reprogrammed hair cells at single cell resolution}

In the Plp ${ }^{\mathrm{CreER+}}$; Atohl-HA+ or Proxl ${ }^{\mathrm{CreER} /+}$; Atohl-HA+ model, because Cre activity is specific to $\mathrm{SCs}$, we are therefore able to distinguish new SC-derived new $\mathrm{HCs}$ from the endogenous HCs by using Atoh1-HA expression as a lineage tracer. In addition, we always found that the Calbindin or Parvalbumin expression in newly reprogrammed $\mathrm{HCs}$ (especially those deriving from IPHs) at P60 was much higher than the adjacent endogenous HCs, which allowed us to definitively identify the new HCs. Indeed, the immuofluorescence of Calbindin or Parvalbumin in the new HCs had to be significantly oversaturated; otherwise it was hard to visualize their expression in the endogenous HCs. We speculated that the high level of Calbindin or Parvalbumin expression in new HCs might be caused by constitutive Atoh1-HA expression.

However, at juvenile ages, the iCre activity of $\mathrm{Fgfr} 3^{i \mathrm{CreER+}}$ mice is not absolutely specific to PCs and DCs ( $2 \%$ of OHCs are also iCre + ); therefore there remains a very low chance that a few Atoh1-HA+/Calbindin+ cells might be endogenous OHCs. In each 160 $\mu \mathrm{m}$ cochlear length, the number $(11 \pm 2, n=3)$ of Atoh1-HA+/ Calbindin + cells was much higher than that of OHCs $(1 \pm 1, \mathrm{n}=3)$ traced by reporter gene EGFP in the $\mathrm{Fgfr} 3^{3 \mathrm{CreER}+}$; $C A G-E G F P+$ mice; Atoh1-HA+/Calbindin+/Prestin-negative cells in OHC regions should be derived from PCs or DCs, because endogenous OHCs should have Prestin at adult ages (unless endogenous OHCs somehow dedifferentiated or downregulated prestin by communicating with PCs or DCs with ectopic Atoh1-HA). Note that iCre activity of $\mathrm{Fg} f 3^{i \mathrm{CrEER}{ }^{+}}$also acted sporadically in a very small fraction of Hensen and Claudius cells. When we defined and quantified HCs, Atoh1-HA+/Calbindin + cells (in LER region) laterally far away from the organ of Corti were not included. It remains possible that some Hensen and Claudius cell-derived Atoh1-HA+/Calbindin+ cells may migrate into the organ of Corti.

\subsubsection{Atoh1 dosage is a determinant factor for the hair cell fate}

Although the Atoh1 gene is required in $\mathrm{HC}$ fate commitment and possibly in the differentiation process,${ }^{38}$ not all Atoh1 expressing cochlear progenitors develop into HCs. ${ }^{36,37,40}$ However, in the Atoh1-EGFP+ transgenic mouse, ${ }^{137}$ EGFP is only detected in HCs. A simple explanation of the different results is that heterogeneous levels of Atoh1 are present between progenitors and $\mathrm{HC}$ lineages (or progenitors who later on commit to HC progenitors). We therefore propose: 1) that, between E12.5 and E14.5, the 
undifferentiated cochlear progenitors have lower levels of Atoh1, and Atoh1 turns on many target genes in progenitors that then permit further cell fate selections, as suggested in cerebellum tissues, ${ }^{140}$ and 2) that after E14.5, the Atoh1 level is somehow dramatically elevated in $\mathrm{HC}$ lineages which will irreversibly differentiate into HCs. ${ }^{39,40}$

Such a hypothesis is further supported by our data that only a small fraction of Atoh1-HA+ SCs turned on HC specific genes and most of the Atoh1-HA+ SCs kept SC fate. In addition, in either Plp ${ }^{\mathrm{CreER}+}$; Atohl-HA+; Atohl-EGFP+ or Fgfr $33^{3^{\text {CreER+ }}{ }^{+} \text {; }}$ Atohl-HA+; Atohl-EGFP+ mice, EGFP+/HA+/Calbindin+ newly generated HCs had high levels of EGFP expression (indicative of endogenous Atoh1 activation). However, Atoh1-HA+/Calbindin- SCs are EGFP-. The Atoh1-EGFP+ is a transgenic line in which only a portion of Atoh1 promoter region, is used to drive EGFP. The recently characterized knock-in Atoh1-EGFP mice ${ }^{141}$ might be required to solidify the endogenous Atoh1 expression pattern in the future. This result also supports that heterogeneous ectopic Atoh1 levels were detected in different Atoh1-HA+ cells. It might account for the fact that only a fraction of SCs could be reprogrammed into HCs, and supports the idea that there is a threshold for ectopic Atoh1-HA level to turn on endogenous Atoh1, only above which can reprogramming occur. It is also consistent with the fact that our other 3 Atoh1-HA transgenic founders cannot drive effective reprogramming. This may be due to the fact that they have overall lower levels of Atoh1 expression related to genome insertion sites or copy numbers.

\subsubsection{Diverse reprogramming pathways among different supporting cells}

In our $P l{ }^{\mathrm{CreER}+}$; Atohl-HA+ model, the IPHs-derived HCs were adjacent (either medial or lateral) to the endogenous IHCs. In many aspects, they resembled the endogenous IHCs of the overall morphology of the entire cell body, shape of the stereocilia with the presence of MET channels, their innervations by spiral ganglia and characteristic patterns of ribbon synaptic structure and the presence of multiple $\mathrm{HC}$ markers. It remains elusive why IPHs-derived HCs prefer the IHC fate and we speculate that it might be correlated to their geographic proximity to spiral ganglion neurons. Furthermore, the reprogramming speeds appear different and unsynchronized among different Atoh1-HA+ IPHs, as the percentage of new HCs increases with post-induction time. It might highlight the heterogeneity among different IPHs. It is possible that the intrinsic differentiation state is different among IPHs, and the less mature IPHs will respond to ectopic Atoh1-HA more rapidly than the more mature ones. Alternatively, it is possible that various dosages of the initial ectopic Atoh1-HA (plus the potential different levels of endogenous Atoh1 that is turned on later) were present in Atoh1-HA+ IPHs, and that reprogramming is faster in cells with the higher net dosage of Atoh1. The various dosages of the initial ectopic Atoh1-HA might be caused by the heterogeneous CAG promoters among different IPHs or the different efficiency of Cre-mediated recombination (or different numbers of floxed stop fragments are deleted if multiple copies of transgene are present) or both. Actually, our southern blot results (by Atoh1 probe) suggest multiple copies of the Atoh1-HA transgene. The more floxed "stop" fragments are deleted, the more copies of Atoh1-HA will be transcribed. 
In our Prox ${ }^{\mathrm{CreER/+}}$; Atoh1-HA+ model, we did not observe new HCs until P22, which was 16 days later than P6 when new HCs were first identified in $\mathrm{Plp}^{\mathrm{CreER+}}$; Atoh1-HA+ model. Why is the reprogramming efficiency higher in the Atoh1-HA+ IPHs? We proposed 3 explanations: 1) The IPHs are more adjacent to GER region, and they might be less mature and more plastic than PCs and DCs; 2) Possible signals such as Notch and Fgfr signaling ${ }^{91}$ are present only in PCs and DCs but not in IPHs that can interfere with the efficiency of Atoh1 in reprogramming PCs and DCs; 3) As supported by the reporter assays, the Cre activity in $P l p^{\mathrm{CreER+}}$ is higher than Prox $1^{\mathrm{CreER/+}}$ mice. More floxed stop fragments can be deleted and more copies of Atoh1-HA will be transcribed, as discussed above.

\subsubsection{Effects of constitutive Atoh1 expression on hair cell differentiation}

Atoh1 is believed to be expressed transiently during the $\mathrm{HC}$ differentiation process and fully differentiated adult HCs do not express Atoh $1 .{ }^{39,40} \mathrm{Such}$ a proposal is further supported by our $\mathrm{Gfil}^{\mathrm{Cre} /+}$; Atohl-HA+ mice. Because the newly generated HCs in $\mathrm{OHC}$ regions were prestin-negative, we first predicted that constitutive overactivation of Atoh1 in embryonic differentiating HCs, especially in OHCs, would interfere with $\mathrm{HC}$ differentiation, with the hypothesis that constitutive Atoh1 expression blocks prestin expression.

In contrast, prestin was present in the surviving OHCs at P14, which suggests that absence of Prestin in new HCs, especially in Prox ${ }^{\mathrm{CreER} /+}$; Atohl-HA+ or Fgfr $3^{\text {iCreER+ }}$; Atohl-HA+, is not caused by constitutive Atoh1 expression. Instead, it is more likely that additional transcriptional factors are needed which can turn on Prestin. Nonetheless, the significant $\mathrm{HC}$ death in $\mathrm{Gfil}^{\mathrm{Cre} /+}$; Atohl-HA+ at P35 clearly showed that constitutive Atoh1 expression is detrimental to the survival of adult HCs. Interestingly, the new HCs can survive till at least P90. Two explanations are proposed: 1) new HCs might not exactly follow the differentiation path (i.e. the global gene expression profiles) of the wild type HCs and somehow better tolerate the constitutive Atoh1;2) Because in Gfil $^{\text {Cre/+ }}$; Atohl-HA+ model, cell death of HCs started from the basal turns (where HCs finish terminal differentiation first) and eventually most HCs died by P35, it is possible that only the adult fully differentiated HCs somehow can sense the aberrant Atoh1 expression and die. If it is true, it is consistent with the notion that our newly generated HCs are not fully matured. Thus, they can survive in the presence of the constitutive Atoh1.

\subsubsection{Overactivating hair cell fate determining and differentiation genes in supporting cells for hair cell regeneration in mammals}

Reprogramming one cell fate to another by overactivating key transcriptional factors is a general and powerful approach for regenerative medicine. SCs in the nonmammalian vertebrates are the sources for regenerating HCs after $\mathrm{HC}$ damage occurs. Atohl is reactivated in the process of $\mathrm{HC}$ regeneration. ${ }^{6}$ To give mammals the capacity to 
regenerate $\mathrm{HCs}$, we tested the effects of constitutive overactivation of Atoh1 in postnatal SCs. Our data clearly supported the concept that high dosage of Atoh1 can reprogram $\mathrm{SCs}$ to adopt the $\mathrm{HC}$ fate without fully differentiation. Therefore, we proposed that high dosage of Atoh1 is sufficient to determine a $\mathrm{HC}$ fate but not to guide the entire process of $\mathrm{HC}$ differentiation. Identifying additional key transcriptional factors that control $\mathrm{HC}$ differentiation, especially which can turn on Prestin, will be informative to understand normal $\mathrm{HC}$ development and $\mathrm{HC}$ regeneration. Thus, combinational overexpression of Atoh1 and these key differentiation genes in SCs will likely generate new HCs which can be fully differentiated.

\subsection{Future Work}

The data described in Figure 5-1 demonstrated that the new HCs derived from IPHs did resemble the endogenous IHCs in many aspects. Thus, one of the most important future work is to determine whether those new HCs are really functional. We plan to choose the highest criterion, which is to damage the endogenous IHCs prior to turning on Atoh1-HA expression in the neonatal IPHs, and then to determine whether those new HCs can compensate loss of the endogenous IHCs.

To achieve such a goal, we have recently characterized a new otoferlin ${ }^{\mathrm{Cr} /+}$ knock-in mouse line in which the otoferlin open reading frame is replaced by Cre (http://www.mmrrc.org/strains/32781/032781.html). By characterizing the otoferlin ${ }^{\mathrm{Cre} /+}$; $C A G-E G F P+$ reporter mouse line, the Cre activity starts to work at $\mathrm{P} 1$ and peaks around P11 (Figure 5-8). Almost all EGFP+ cells were IHCs at P1. Interestingly, much more EGFP+ IHCs could be observed in basal and middle turns than in apical turns (Figure 5-8A-C). At P11, the distribution of EGFP+ HCs was similar among different turns, and $\sim 95 \%$ IHCs were EGFP+, and $\sim 10 \%$ OHCs were EGFP+. The significantly enriched Cre activity in IHCs of otoferlin ${ }^{\mathrm{Cre} / \mathrm{t}}$ mice allows us to use this line to damage the IHCs, while leaving OHCs intact.

$$
\mathrm{Plp}^{\mathrm{CreER}{ }^{+}} \text {; otoferlin }{ }_{\text {Crel+ }} \text {; Rosa26-loxp-stop-loxp-rtTA/+; TetO-DTA+; Atoh1-HA+ }
$$

mouse line will be used as experimental group to test the functionality of the new HCs deriving from IPHs, while $\mathrm{Plp}^{\text {CreER+ }}$; otoferlin ${ }^{\mathrm{Cre} /+} ;$ Rosa26-loxp-stop-loxp-rtTA/+; TetO-DTA+ mouse line will be used as control group. As illustrated in Figure 5-9, we planned to give Doxycycline containing food to both experimental and control group at $\sim$ E16 or E18 (precise time will be finalized after further preliminary experiments). Then, doxycycline containing food will be stopped for 3 days (hopefully doxycycline concentration can be decreased below the working threshold within 3 days), after which mice will be treated with tamoxifen around $\mathrm{P} 3$ and $\mathrm{P} 4$ (to turn on Atoh1-HA) and their hearing capacities will be determined by our ABR measurement. If those new HCs are functional, the experimental group mice should have better hearing ability than the control group. 


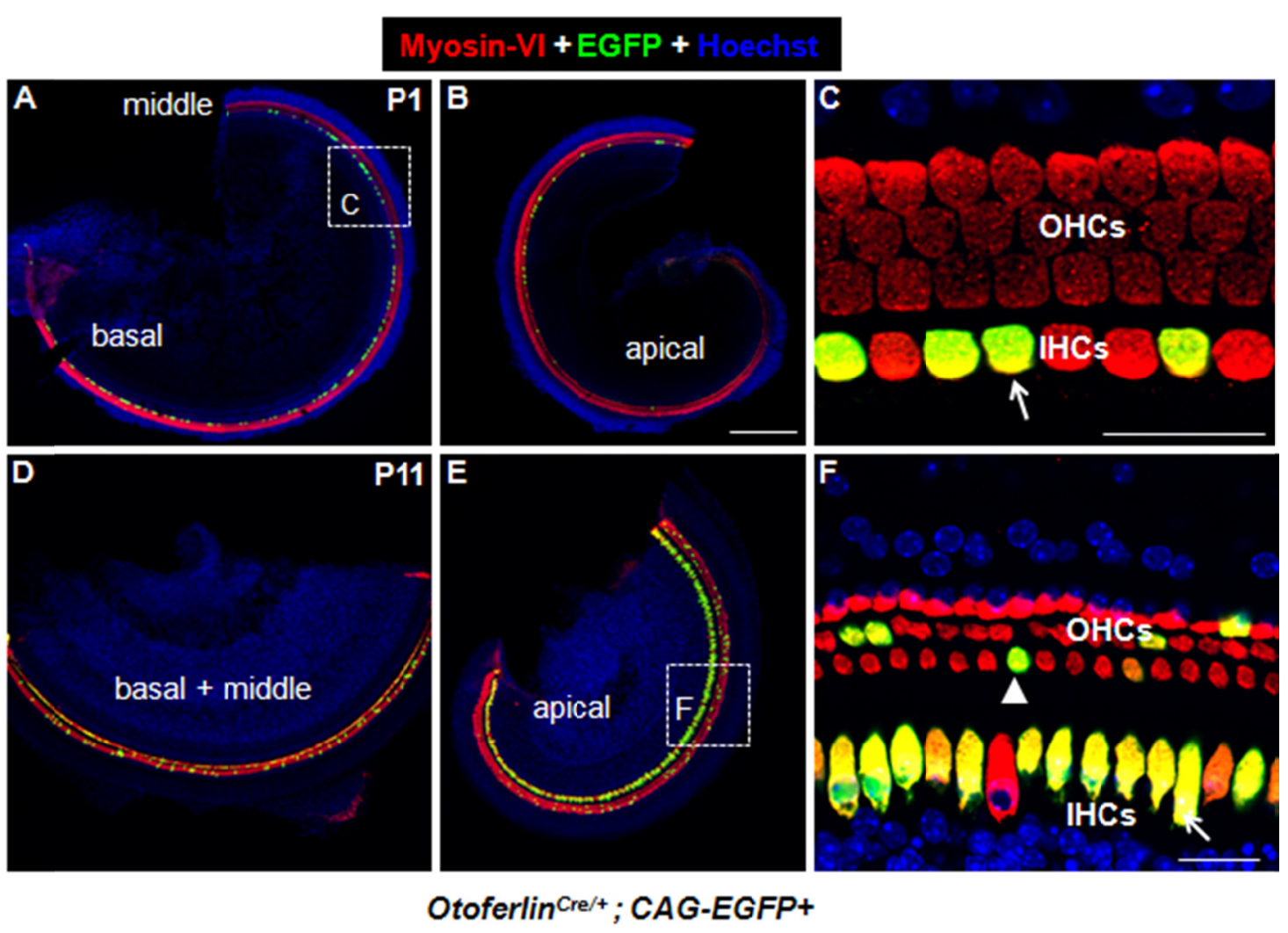

Figure 5-8 Characterization of recombinase Cre activity in Otoferlin ${ }^{\mathrm{Cre} /+}$ mice. (A-C) Double staining of Myosin-VI and EGFP of cochlear samples at P1. (C) is the high magnification image of the square region in (A). Arrow labels one EGFP+ IHC. (D-F) Double staining of Myosin-VI and EGFP of cochlear samples at P11. (F) is the high magnification image of the square region in (E). Arrow labels one EGFP+ IHC and arrow head labels one EGFP+ OHCs. Bars: $200 \mu \mathrm{m}$ in (B), $20 \mu \mathrm{m}$ in (C, F). 


\section{Experimental}

Otoferlin-Cre/+;

R26-flox-stop-flox-rtTA+;

TetO-DTA+;

PLP-CREER+;

Atoh1- flox-stop-flox-HA +;

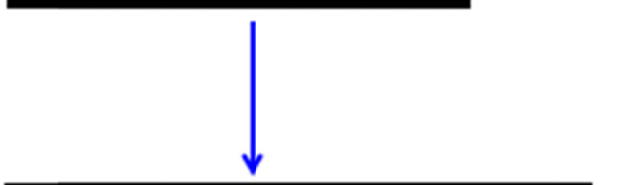

Otoferlin-Cre/+;

R26-rTTA+;

TetO-DTA+ (in IHCs and died);

PLP-CREER+;

Atoh1-HA+ (in IHCs and died),

Atoh1- flox-stop-flox-HA+ (in IPHs);

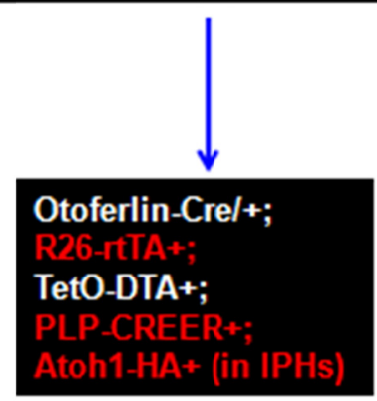

New HCs
Control

Otoferlin-Cre/+;

R26-flox-stop-flox-rtTA+;

TetO-DTA+;

PLP-CREER+;

Dox+ food

(E16-P1)

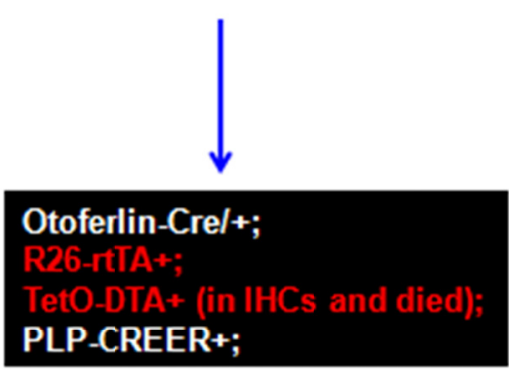

Stop Dox+ food

Tamoxifen at P4

\section{Otoferlin-Cre/+;}

R26-rTA+;

TetO-DTA+;

PLP-CREER+;

No new HCs

Figure 5-9 Experimental strategy for determining the functionality of the new hair cells deriving from inner phalangeal cells.

The left panel is the experimental group where endogenous IHCs will be damaged first by DTA before P4. DTA will cause cell death in a cell-autonomous manner. Following cell death of the endogenous IHCs, tamoxifen treatment at P4 will turn on ectopic Atoh1 in IPHs. The right panel is the control group where endogenous IHCs will be damaged first by DTA before P4 but no new HCs will be generated. If the experimental group mice have better hearing capacity than control group mice, it will strongly prove that the new HCs are functional. 


\section{CHAPTER 6. PROSPECTIVE FOR MAMMALIAN HAIR CELL REGENERATION}

\subsection{Introduction}

Our data described in previous chapters provide foundations for mammalian hair cell regeneration. To mimic the $\mathrm{HC}$ regeneration mechanism used in non-mammalian vertebrates, inactivation of either Sox 2 or $\mathrm{p} 27^{\mathrm{Kip} 1}$ can be used to induce SC subtypes to proliferate, and ectopic expression of Atoh1 can be utilized to reprogram SCs into HCs. It is worthy to emphasize that both scenarios only occur in mouse cochleae at young ages, but not adult ages. Unfortunately, it remains unclear why adult SCs become insensitive to the manipulation of Sox2, p2 $7^{\text {Kip } 1}$ and Atoh1. Furthermore, the neonatal or juvenile ages in mice correspond to the end of the first trimester/beginning of the second trimester, or within the second trimester of human embryogenesis, respectively. The comparison between mice and human is based on the information of website (http://www.translatingtime.net/). Briefly, the translational information is built on the Finlay/Darlington model, ${ }^{142,143}$ in which three factors, species factor, neural events factor and primate factor, are used to adjust the differences among different species (primates or non-primates), or different organs (cortical regions, limbic systems and non-cortical, non-limbic regions). The primate factor only applies to primates $(0.248683$ for cortical event, and -0.079280 for limbic event). Therefore, it is critical to study how to achieve reprogramming of adult mouse SCs to HCs, and to figure out why the adult SCs have declined proliferative capacities. Our final goal of will be to heal human deafness which primarily occurs at postnatal ages by various kinds of ototoxic factors.

\subsection{Conversion of Adult Cochlear Cells into Young and Plastic Ages}

With the advancement of cellular differentiation, each individual cell becomes committed to their own gene expression and unique functions. The mechanisms underlying these changes along the cellular maturation process are referred as to epigenetic. Developmental ages in many cases determine the cellular responses to overactivation or inactivation of genes, which is further supported by the recent studies in hematopoietic ${ }^{144}$ or pancreatic cell lineages. ${ }^{145}$ While the detailed mechanism underlying the different reprogramming efficient of the same lineage at different ages remains unclear, distinct status DNA acetylation or methylation is suggested to play critical roles. $^{146,147}$

In terms of how to achieve the goal of reprogramming the adult cochlear SCs into $\mathrm{HCs}$ for mammalian $\mathrm{HC}$ regeneration, as suggested by recent studies in the regenerative medicine field, ${ }^{144}$ one applicable approach is in vivo overactivating the four iPS (induced pluripotent stem cells) factors into the adult SCs (P30). ${ }^{148}$ As shown in Fig. 6, if adult SCs with ectopic expression of the four iPS factors respond to Atoh1 overactivation and are reprogrammed into $\mathrm{HCs}$, it will suggest that the four iPS factors are sufficient to convert adult cochlear SCs into the neonatal or juvenile ages that normally respond to the 
overactivation of Atoh1. This is clearly of particular interest in adult human HC regeneration that mainly occurs at aged human. In addition, it also will provide a great model to further figure out the epigenetic changes in adult SCs between with and without overactivation of four of iPS factors. However, currently there is no direct evidence to suggest why the adult SCs, compared with neonatal and juvenile SCs, have significantly declined proliferative capacities. Further studies are needed that aim at elucidating the signaling pathways promoting the proliferation of SCs.

\subsection{Application of Mouse Pre-clinical Models to Humans}

The goal of studies using genetically engineered mouse models is to provide preclinical information for clinical medicine in humans. Different from mouse studies in which genome DNA sequence are altered for various purposes, the ideal way or treating deaf patients, besides cochlear explants transplantation, could be the use of small molecules-derived drugs. These drugs roughly can be categorized into two types. The first are drugs that can promote proliferation of cells including cochlear SCs. The second are the modifiers of chromatin structure, or drugs that promote transcription of $\mathrm{HC}$ specific genes, especially Atoh1.

Drugs that promote cell proliferation are beneficial for generating new HCs, but on the other hand, they are tumorigenic especially in the fast growing organs such as intestine and stomach. To avoid the systemic side effects, the best way would be transient local delivery through cochlear oval window exposed toward the middle ear. If systemic delivery is required for some drugs to be active (i.e. needs modifications by enzymes in liver), it might still be possible to transiently use them in patients whose deafness is triggered by chemotherapy to treat pre-existing tumors (i.e. children with ALL).

The second type of drugs ideal for converting human SCs into HCs should be able to reprogram adult mouse $\mathrm{SCs}$ into $\mathrm{HCs}$, with consideration of the translational developmental events between mice and human. One type of drug here activates Atoh1 at the transcriptional level because Atoh1 normally is inactive in adult mouse SCs. ${ }^{39,40}$ To screen for such a drug in a high-throughput manner, mouse embryonic fibroblast cells (MEFs) isolated from Arf ${ }^{/-}$; Atoh $1^{\text {EGFP/EGFP }}$ double mutant mice. Lack of Arf (alternative reading frame) tumor suppressor gene makes MEFs immotile, thus growing rapidly in vitro. Atoh $1^{E G F P / E G F P}$ is a knock-in mouse model in which the EGFP coding sequence is tagged at the 3' end of Atoh1 gene. ${ }^{141}$ MEFs will be treated with different small molecules and turning on EGFP will indicate activation of Atoh1. Thus, EGFP will be used as read-out to judge what small molecules should be used in secondary assays in inner ear cells. Other drugs might be those that are able to promote efficiency of reprogramming. However, preliminary epigenetic comparison data between adult SCs with or without four ectopic iPS factors are needed, with the assumption that adult SCs with ectopic four iPS factors will respond to ectopic Atoh1 expression. These drugs might be modifier of chromatin structure. 
A

Drugs

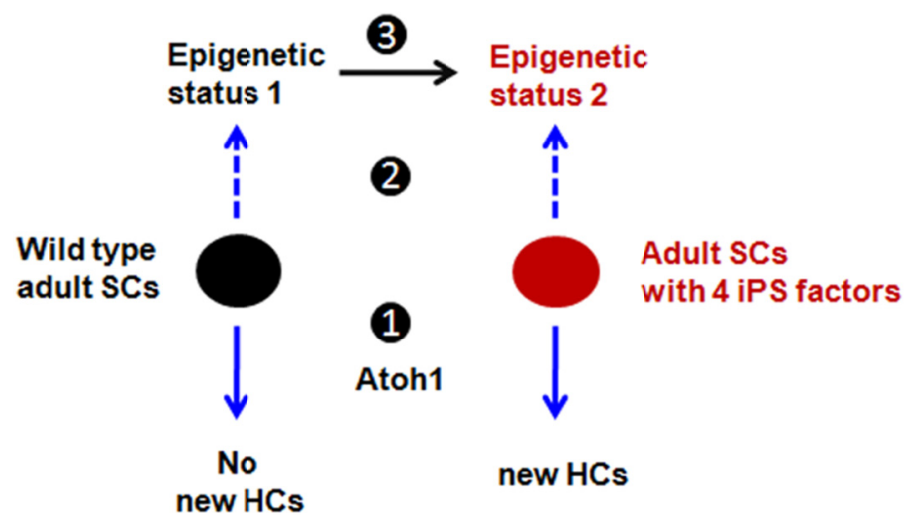

B

\section{Art $\%$; Atoh1EGFPIEGFP mice}

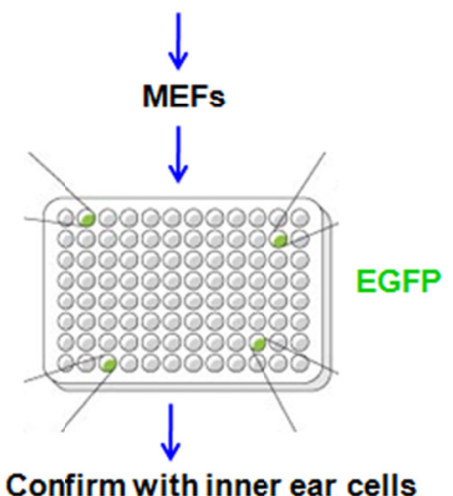

Figure 6-1 Screening drugs for translational study in human HC regeneration. (A) Three steps toward probing candidate drugs. In step 1, different responsive between two kinds of adult SCs to Atoh1 is used to demonstrate the activities of 4 iPS factors. Thus, two different epigenetic states are determined in step 2. Drugs that are able to promote transition from epigenetic status 1 to 2 are screened as candidate drugs. (B) MEF cells produced from $\mathrm{Arf}^{-}$; Atoh $1^{\text {EGFP/EGFP }}$ mice will be treated with different drugs and EGFP+ wells indicate that drugs inside can promote Atoh1 transcription. 


\section{LIST OF REFERENCES}

1. Corwin JT, Cotanche DA. Regeneration of sensory hair cells after acoustic trauma. Science 1988; 240:1772-4.

2. Cruz RM, Lambert PR, Rubel EW. Light microscopic evidence of hair cell regeneration after gentamicin toxicity in chick cochlea. Arch Otolaryngol Head Neck Surg 1987; 113:1058-62.

3. Cotanche DA. Regeneration of hair cell stereociliary bundles in the chick cochlea following severe acoustic trauma. Hear Res 1987; 30:181-95.

4. Stone JS, Cotanche DA. Hair cell regeneration in the avian auditory epithelium. Int J Dev Biol 2007; 51:633-47.

5. Ryals BM, Rubel EW. Hair cell regeneration after acoustic trauma in adult Coturnix quail. Science 1988; 240:1774-6.

6. Cafaro J, Lee GS, Stone JS. Atoh1 expression defines activated progenitors and differentiating hair cells during avian hair cell regeneration. Dev Dyn 2007; 236:156-70.

7. Fekete DM, Muthukumar S, Karagogeos D. Hair cells and supporting cells share a common progenitor in the avian inner ear. J Neurosci 1998; 18:7811-21.

8. Kopan R, Ilagan MX. The canonical Notch signaling pathway: unfolding the activation mechanism. Cell 2009; 137:216-33.

9. Morrison A, Hodgetts C, Gossler A, Hrabe de Angelis M, Lewis J. Expression of Delta1 and Serrate1 (Jagged1) in the mouse inner ear. Mech Dev 1999; 84:16972.

10. Lewis AK, Frantz GD, Carpenter DA, de Sauvage FJ, Gao WQ. Distinct expression patterns of notch family receptors and ligands during development of the mammalian inner ear. Mech Dev 1998; 78:159-63. 
11. Luo B, Aster JC, Hasserjian RP, Kuo F, Sklar J. Isolation and functional analysis of a cDNA for human Jagged2, a gene encoding a ligand for the Notch1 receptor. Mol Cell Biol 1997; 17:6057-67.

12. Hartman BH, Hayashi T, Nelson BR, Bermingham-McDonogh O, Reh TA. Dl13 is expressed in developing hair cells in the mammalian cochlea. Dev Dyn 2007; 236:2875-83.

13. Pan W, Jin Y, Stanger B, Kiernan AE. Notch signaling is required for the generation of hair cells and supporting cells in the mammalian inner ear. Proc Natl Acad Sci U S A 2010; 107:15798-803.

14. Kiernan AE, Cordes R, Kopan R, Gossler A, Gridley T. The Notch ligands DLL1 and JAG2 act synergistically to regulate hair cell development in the mammalian inner ear. Development 2005; 132:4353-62.

15. Hartman BH, Reh TA, Bermingham-McDonogh O. Notch signaling specifies prosensory domains via lateral induction in the developing mammalian inner ear. Proc Natl Acad Sci U S A 2010; 107:15792-7.

16. Brooker R, Hozumi K, Lewis J. Notch ligands with contrasting functions: Jagged1 and Delta1 in the mouse inner ear. Development 2006; 133:1277-86.

17. Kiernan AE, Xu J, Gridley T. The Notch ligand JAG1 is required for sensory progenitor development in the mammalian inner ear. PLoS Genet 2006; 2:e4.

18. Yamamoto N, Chang W, Kelley MW. Rbpj regulates development of prosensory cells in the mammalian inner ear. Dev Biol 2011; 353:367-79.

19. Tsai H, Hardisty RE, Rhodes C, Kiernan AE, Roby P, Tymowska-Lalanne Z, Mburu P, Rastan S, Hunter AJ, Brown SD, Steel KP. The mouse slalom mutant demonstrates a role for Jagged 1 in neuroepithelial patterning in the organ of Corti. Hum Mol Genet 2001; 10:507-12.

20. Daudet N, Lewis J. Two contrasting roles for Notch activity in chick inner ear development: specification of prosensory patches and lateral inhibition of hair-cell differentiation. Development 2005; 132:541-51. 
21. Hayashi T, Kokubo H, Hartman BH, Ray CA, Reh TA, Bermingham-McDonogh O. Hesrl and Hesr2 may act as early effectors of Notch signaling in the developing cochlea. Dev Biol 2008; 316:87-99.

22. Eddison M, Le Roux I, Lewis J. Notch signaling in the development of the inner ear: lessons from Drosophila. Proc Natl Acad Sci U S A 2000; 97:11692-9.

23. Zhang N, Martin GV, Kelley MW, Gridley T. A mutation in the Lunatic fringe gene suppresses the effects of a Jagged2 mutation on inner hair cell development in the cochlea. Curr Biol 2000; 10:659-62.

24. Lanford PJ, Lan Y, Jiang R, Lindsell C, Weinmaster G, Gridley T, Kelley MW. Notch signalling pathway mediates hair cell development in mammalian cochlea. Nat Genet 1999; 21:289-92.

25. Haddon C, Jiang YJ, Smithers L, Lewis J. Delta-Notch signalling and the patterning of sensory cell differentiation in the zebrafish ear: evidence from the mind bomb mutant. Development 1998; 125:4637-44.

26. Tateya T, Imayoshi I, Tateya I, Ito J, Kageyama R. Cooperative functions of Hes/Hey genes in auditory hair cell and supporting cell development. Dev Biol $2011 ; 352: 329-40$.

27. Liu Z, Zuo J. Cell cycle regulation in hair cell development and regeneration in the mouse cochlea. Cell Cycle 2008; 7:2129-33.

28. Zine A, Aubert A, Qiu J, Therianos S, Guillemot F, Kageyama R, de Ribaupierre $\mathrm{F}$. Hes1 and Hes5 activities are required for the normal development of the hair cells in the mammalian inner ear. J Neurosci 2001; 21:4712-20.

29. Zheng JL, Shou J, Guillemot F, Kageyama R, Gao WQ. Hes 1 is a negative regulator of inner ear hair cell differentiation. Development 2000; 127:4551-60.

30. Jarman AP, Grau Y, Jan LY, Jan YN. atonal is a proneural gene that directs chordotonal organ formation in the Drosophila peripheral nervous system. Cell 1993; 73:1307-21. 
31. Jarman AP, Sun Y, Jan LY, Jan YN. Role of the proneural gene, atonal, in formation of Drosophila chordotonal organs and photoreceptors. Development 1995; 121:2019-30.

32. Ben-Arie N, Bellen HJ, Armstrong DL, McCall AE, Gordadze PR, Guo Q, Matzuk MM, Zoghbi HY. Math1 is essential for genesis of cerebellar granule neurons. Nature 1997; 390:169-72.

33. Yang Q, Bermingham NA, Finegold MJ, Zoghbi HY. Requirement of Math1 for secretory cell lineage commitment in the mouse intestine. Science 2001; 294:2155-8.

34. Ben-Arie N, McCall AE, Berkman S, Eichele G, Bellen HJ, Zoghbi HY. Evolutionary conservation of sequence and expression of the bHLH protein Atonal suggests a conserved role in neurogenesis. Hum Mol Genet 1996; 5:120716.

35. Du X, Jensen P, Goldowitz D, Hamre KM. Wild-type cells rescue genotypically Math1-null hair cells in the inner ears of chimeric mice. Dev Biol 2007; 305:4308.

36. Yang H, Xie X, Deng M, Chen X, Gan L. Generation and characterization of Atoh1-Cre knock-in mouse line. Genesis 2010; 48:407-13.

37. Matei V, Pauley S, Kaing S, Rowitch D, Beisel KW, Morris K, Feng F, Jones K, Lee J, Fritzsch B. Smaller inner ear sensory epithelia in Neurog 1 null mice are related to earlier hair cell cycle exit. Dev Dyn 2005; 234:633-50.

38. Bermingham NA, Hassan BA, Price SD, Vollrath MA, Ben-Arie N, Eatock RA, Bellen HJ, Lysakowski A, Zoghbi HY. Math1: an essential gene for the generation of inner ear hair cells. Science 1999; 284:1837-41.

39. Chow LM, Tian Y, Weber T, Corbett M, Zuo J, Baker SJ. Inducible Cre recombinase activity in mouse cerebellar granule cell precursors and inner ear hair cells. Dev Dyn 2006; 235:2991-8.

40. Woods C, Montcouquiol M, Kelley MW. Math1 regulates development of the sensory epithelium in the mammalian cochlea. Nat Neurosci 2004; 7:1310-8. 
41. Gubbels SP, Woessner DW, Mitchell JC, Ricci AJ, Brigande JV. Functional auditory hair cells produced in the mammalian cochlea by in utero gene transfer. Nature 2008; 455:537-41.

42. Izumikawa M, Minoda R, Kawamoto K, Abrashkin KA, Swiderski DL, Dolan DF, Brough DE, Raphael Y. Auditory hair cell replacement and hearing improvement by Atoh1 gene therapy in deaf mammals. Nat Med 2005; 11:271-6.

43. Zheng JL, Gao WQ. Overexpression of Math1 induces robust production of extra hair cells in postnatal rat inner ears. Nat Neurosci 2000; 3:580-6.

44. Kawamoto K, Ishimoto S, Minoda R, Brough DE, Raphael Y. Math1 gene transfer generates new cochlear hair cells in mature guinea pigs in vivo. J Neurosci 2003; 23:4395-400.

45. Kamachi Y, Uchikawa M, Kondoh H. Pairing SOX off: with partners in the regulation of embryonic development. Trends Genet 2000; 16:182-7.

46. Kiernan AE, Pelling AL, Leung KK, Tang AS, Bell DM, Tease C, Lovell-Badge $\mathrm{R}$, Steel KP, Cheah KS. Sox2 is required for sensory organ development in the mammalian inner ear. Nature 2005; 434:1031-5.

47. Puligilla C, Dabdoub A, Brenowitz SD, Kelley MW. Sox2 induces neuronal formation in the developing mammalian cochlea. J Neurosci 2010; 30:714-22.

48. Dabdoub A, Puligilla C, Jones JM, Fritzsch B, Cheah KS, Pevny LH, Kelley MW. Sox2 signaling in prosensory domain specification and subsequent hair cell differentiation in the developing cochlea. Proc Natl Acad Sci U S A 2008; 105:18396-401.

49. Mak AC, Szeto IY, Fritzsch B, Cheah KS. Differential and overlapping expression pattern of SOX2 and SOX9 in inner ear development. Gene Expr Patterns 2009; 9:444-53.

50. Hume CR, Bratt DL, Oesterle EC. Expression of LHX3 and SOX2 during mouse inner ear development. Gene Expr Patterns 2007; 7:798-807. 
51. Oesterle EC, Campbell S, Taylor RR, Forge A, Hume CR. Sox2 and JAGGED1 expression in normal and drug-damaged adult mouse inner ear. J Assoc Res Otolaryngol 2008; 9:65-89.

52. Taranova OV, Magness ST, Fagan BM, Wu Y, Surzenko N, Hutton SR, Pevny LH. SOX2 is a dose-dependent regulator of retinal neural progenitor competence. Genes Dev 2006; 20:1187-202.

53. Favaro R, Valotta M, Ferri AL, Latorre E, Mariani J, Giachino C, Lancini C, Tosetti V, Ottolenghi S, Taylor V, Nicolis SK. Hippocampal development and neural stem cell maintenance require Sox2-dependent regulation of Shh. Nat Neurosci 2009; 12:1248-56.

54. Okubo T, Pevny LH, Hogan BL. Sox2 is required for development of taste bud sensory cells. Genes Dev 2006; 20:2654-9.

55. Que J, Luo X, Schwartz RJ, Hogan BL. Multiple roles for Sox2 in the developing and adult mouse trachea. Development 2009; 136:1899-907.

56. Sherr CJ, Roberts JM. CDK inhibitors: positive and negative regulators of G1-phase progression. Genes Dev 1999; 13:1501-12.

57. Fero ML, Rivkin M, Tasch M, Porter P, Carow CE, Firpo E, Polyak K, Tsai LH, Broudy V, Perlmutter RM, Kaushansky K, Roberts JM. A syndrome of multiorgan hyperplasia with features of gigantism, tumorigenesis, and female sterility in p27(Kip1)-deficient mice. Cell 1996; 85:733-44.

58. Nakayama K, Ishida N, Shirane M, Inomata A, Inoue T, Shishido N, Horii I, Loh DY. Mice lacking p27(Kip1) display increased body size, multiple organ hyperplasia, retinal dysplasia, and pituitary tumors. Cell 1996; 85:707-20.

59. Kiyokawa H, Kineman RD, Manova-Todorova KO, Soares VC, Hoffman ES, Ono M, Khanam D, Hayday AC, Frohman LA, Koff A. Enhanced growth of mice lacking the cyclin-dependent kinase inhibitor function of p27(Kip1). Cell 1996; 85:721-32.

60. Besson A, Dowdy SF, Roberts JM. CDK inhibitors: cell cycle regulators and beyond. Dev Cell 2008; 14:159-69. 
61. Besson A, Hwang HC, Cicero S, Donovan SL, Gurian-West M, Johnson D, Clurman BE, Dyer MA, Roberts JM. Discovery of an oncogenic activity in p27Kip1 that causes stem cell expansion and a multiple tumor phenotype. Genes Dev 2007; 21:1731-46.

62. Lee YS, Liu F, Segil N. A morphogenetic wave of p27Kip1 transcription directs cell cycle exit during organ of Corti development. Development 2006; 133:281726.

63. Kelley MW. Regulation of cell fate in the sensory epithelia of the inner ear. Nat Rev Neurosci 2006; 7:837-49.

64. Chen P, Segil N. p27(Kip1) links cell proliferation to morphogenesis in the developing organ of Corti. Development 1999; 126:1581-90.

65. Lowenheim H, Furness DN, Kil J, Zinn C, Gultig K, Fero ML, Frost D, Gummer AW, Roberts JM, Rubel EW, Hackney CM, Zenner HP. Gene disruption of p27(Kip1) allows cell proliferation in the postnatal and adult organ of corti. Proc Natl Acad Sci U S A 1999; 96:4084-8.

66. White PM, Doetzlhofer A, Lee YS, Groves AK, Segil N. Mammalian cochlear supporting cells can divide and trans-differentiate into hair cells. Nature 2006; 441:984-7.

67. Gao J, Wu X, Zuo J. Targeting hearing genes in mice. Brain Res Mol Brain Res 2004; 132:192-207.

68. Sauer B, Henderson N. Site-specific DNA recombination in mammalian cells by the Cre recombinase of bacteriophage P1. Proc Natl Acad Sci U S A 1988; 85:5166-70.

69. Sternberg N, Hamilton D, Austin S, Yarmolinsky M, Hoess R. Site-specific recombination and its role in the life cycle of bacteriophage P1. Cold Spring Harb Symp Quant Biol 1981; 45 Pt 1:297-309.

70. Joyner AL, Zervas M. Genetic inducible fate mapping in mouse: establishing genetic lineages and defining genetic neuroanatomy in the nervous system. Dev Dyn 2006; 235:2376-85. 
71. Nagy A. Cre recombinase: the universal reagent for genome tailoring. Genesis 2000; 26:99-109.

72. Chien WM, Rabin S, Macias E, Miliani de Marval PL, Garrison K, Orthel J, Rodriguez-Puebla M, Fero ML. Genetic mosaics reveal both cell-autonomous and cell-nonautonomous function of murine p27Kip1. Proc Natl Acad Sci U S A 2006; 103:4122-7.

73. Yu Y, Weber T, Yamashita T, Liu Z, Valentine MB, Cox BC, Zuo J. In vivo proliferation of postmitotic cochlear supporting cells by acute ablation of the retinoblastoma protein in neonatal mice. J Neurosci 2010; 30:5927-36.

74. Young KM, Mitsumori T, Pringle N, Grist M, Kessaris N, Richardson WD. An Fgfr3-iCreER(T2) transgenic mouse line for studies of neural stem cells and astrocytes. Glia 2010; 58:943-53.

75. Rivers LE, Young KM, Rizzi M, Jamen F, Psachoulia K, Wade A, Kessaris N, Richardson WD. PDGFRA/NG2 glia generate myelinating oligodendrocytes and piriform projection neurons in adult mice. Nat Neurosci 2008; 11:1392-401.

76. Srinivasan RS, Dillard ME, Lagutin OV, Lin FJ, Tsai S, Tsai MJ, Samokhvalov IM, Oliver G. Lineage tracing demonstrates the venous origin of the mammalian lymphatic vasculature. Genes Dev 2007; 21:2422-32.

77. Wu X, Gao J, Guo Y, Zuo J. Hearing threshold elevation precedes hair-cell loss in prestin knockout mice. Brain Res Mol Brain Res 2004; 126:30-7.

78. Salic A, Mitchison TJ. A chemical method for fast and sensitive detection of DNA synthesis in vivo. Proc Natl Acad Sci U S A 2008; 105:2415-20.

79. Jacques BE, Montcouquiol ME, Layman EM, Lewandoski M, Kelley MW. Fgf8 induces pillar cell fate and regulates cellular patterning in the mammalian cochlea. Development 2007; 134:3021-9.

80. Zindy F, Uziel T, Ayrault O, Calabrese C, Valentine M, Rehg JE, Gilbertson RJ, Sherr CJ, Roussel MF. Genetic alterations in mouse medulloblastomas and generation of tumors de novo from primary cerebellar granule neuron precursors. Cancer Res 2007; 67:2676-84. 
81. Stone JS, Rubel EW. Deltal expression during avian hair cell regeneration. Development 1999; 126:961-73.

82. Brignull HR, Raible DW, Stone JS. Feathers and fins: non-mammalian models for hair cell regeneration. Brain Res 2009; 1277:12-23.

83. Bermingham-McDonogh O, Oesterle EC, Stone JS, Hume CR, Huynh HM, Hayashi T. Expression of Prox1 during mouse cochlear development. J Comp Neurol 2006; 496:172-86.

84. Dyer MA, Cepko CL. p27Kip1 and p57Kip2 regulate proliferation in distinct retinal progenitor cell populations. J Neurosci 2001; 21:4259-71.

85. Dyer MA, Cepko CL. The p57Kip2 cyclin kinase inhibitor is expressed by a restricted set of amacrine cells in the rodent retina. J Comp Neurol 2001; 429:60114.

86. Chen P, Zindy F, Abdala C, Liu F, Li X, Roussel MF, Segil N. Progressive hearing loss in mice lacking the cyclin-dependent kinase inhibitor Ink4d. Nat Cell Biol 2003; 5:422-6.

87. Weber T, Corbett MK, Chow LM, Valentine MB, Baker SJ, Zuo J. Rapid cell-cycle reentry and cell death after acute inactivation of the retinoblastoma gene product in postnatal cochlear hair cells. Proc Natl Acad Sci U S A 2008; 105:781-5.

88. Sage C, Huang M, Vollrath MA, Brown MC, Hinds PW, Corey DP, Vetter DE, Chen ZY. Essential role of retinoblastoma protein in mammalian hair cell development and hearing. Proc Natl Acad Sci U S A 2006; 103:7345-50.

89. Laine H, Doetzlhofer A, Mantela J, Ylikoski J, Laiho M, Roussel MF, Segil N, Pirvola U. p19(Ink4d) and p21(Cip1) collaborate to maintain the postmitotic state of auditory hair cells, their codeletion leading to DNA damage and p53-mediated apoptosis. J Neurosci 2007; 27:1434-44.

90. Ono K, Nakagawa T, Kojima K, Matsumoto M, Kawauchi T, Hoshino M, Ito J. Silencing p27 reverses post-mitotic state of supporting cells in neonatal mouse cochleae. Mol Cell Neurosci 2009; 42:391-8. 
91. Doetzlhofer A, Basch ML, Ohyama T, Gessler M, Groves AK, Segil N. Hey2 regulation by FGF provides a Notch-independent mechanism for maintaining pillar cell fate in the organ of Corti. Dev Cell 2009; 16:58-69.

92. Avilion AA, Nicolis SK, Pevny LH, Perez L, Vivian N, Lovell-Badge R. Multipotent cell lineages in early mouse development depend on SOX2 function. Genes Dev 2003; 17:126-40.

93. Miyagi S, Masui S, Niwa H, Saito T, Shimazaki T, Okano H, Nishimoto M, Muramatsu M, Iwama A, Okuda A. Consequence of the loss of Sox2 in the developing brain of the mouse. FEBS Lett 2008; 582:2811-5.

94. Laine H, Sulg M, Kirjavainen A, Pirvola U. Cell cycle regulation in the inner ear sensory epithelia: role of cyclin D1 and cyclin-dependent kinase inhibitors. Dev Biol 2009; 337:134-46.

95. Minami S, Ohtani-Fujita N, Igata E, Tamaki T, Sakai T. Molecular cloning and characterization of the human p27Kip1 gene promoter. FEBS Lett 1997; 411:1-6.

96. Chen X, Xu H, Yuan P, Fang F, Huss M, Vega VB, Wong E, Orlov YL, Zhang W, Jiang J, Loh YH, Yeo HC, Yeo ZX, Narang V, Govindarajan KR, Leong B, Shahab A, Ruan Y, Bourque G, Sung WK, Clarke ND, Wei CL, Ng HH. Integration of external signaling pathways with the core transcriptional network in embryonic stem cells. Cell 2008; 133:1106-17.

97. Marson A, Levine SS, Cole MF, Frampton GM, Brambrink T, Johnstone S, Guenther MG, Johnston WK, Wernig M, Newman J, Calabrese JM, Dennis LM, Volkert TL, Gupta S, Love J, Hannett N, Sharp PA, Bartel DP, Jaenisch R, Young RA. Connecting microRNA genes to the core transcriptional regulatory circuitry of embryonic stem cells. Cell 2008; 134:521-33.

98. Breuskin I, Bodson M, Thelen N, Thiry M, Borgs L, Nguyen L, Lefebvre PP, Malgrange B. Sox 10 promotes the survival of cochlear progenitors during the establishment of the organ of Corti. Dev Biol 2009; 335:327-39.

99. Behra M, Bradsher J, Sougrat R, Gallardo V, Allende ML, Burgess SM. Phoenix is required for mechanosensory hair cell regeneration in the zebrafish lateral line. PLoS Genet 2009; 5:e1000455. 
100. Imayoshi I, Sakamoto M, Yamaguchi M, Mori K, Kageyama R. Essential roles of Notch signaling in maintenance of neural stem cells in developing and adult brains. J Neurosci 2010; 30:3489-98.

101. Fekete DM, Wu DK. Revisiting cell fate specification in the inner ear. Curr Opin Neurobiol 2002; 12:35-42.

102. Raft S, Koundakjian EJ, Quinones H, Jayasena CS, Goodrich LV, Johnson JE, Segil N, Groves AK. Cross-regulation of Ngn1 and Math1 coordinates the production of neurons and sensory hair cells during inner ear development. Development 2007; 134:4405-15.

103. Jahan I, Pan N, Kersigo J, Fritzsch B. Neurod1 suppresses hair cell differentiation in ear ganglia and regulates hair cell subtype development in the cochlea. PLoS One 2010; 5:e11661.

104. Murtaugh LC, Stanger BZ, Kwan KM, Melton DA. Notch signaling controls multiple steps of pancreatic differentiation. Proc Natl Acad Sci U S A 2003; 100:14920-5.

105. Rawlins EL, Clark CP, Xue Y, Hogan BL. The Id2+ distal tip lung epithelium contains individual multipotent embryonic progenitor cells. Development 2009; 136:3741-5.

106. Neves J, Parada C, Chamizo M, Giraldez F. Jagged 1 regulates the restriction of Sox2 expression in the developing chicken inner ear: a mechanism for sensory organ specification. Development 2011; 138:735-44.

107. Jadhav AP, Cho SH, Cepko CL. Notch activity permits retinal cells to progress through multiple progenitor states and acquire a stem cell property. Proc Natl Acad Sci U S A 2006; 103:18998-9003.

108. Vooijs M, Ong CT, Hadland B, Huppert S, Liu Z, Korving J, van den Born M, Stappenbeck T, Wu Y, Clevers H, Kopan R. Mapping the consequence of Notch1 proteolysis in vivo with NIP-CRE. Development 2007; 134:535-44.

109. Conlon RA, Reaume AG, Rossant J. Notch1 is required for the coordinate segmentation of somites. Development 1995; 121:1533-45. 
110. Swiatek PJ, Lindsell CE, del Amo FF, Weinmaster G, Gridley T. Notch1 is essential for postimplantation development in mice. Genes Dev 1994; 8:707-19.

111. Basch ML, Ohyama T, Segil N, Groves AK. Canonical Notch Signaling Is Not Necessary for Prosensory Induction in the Mouse Cochlea: Insights from a Conditional Mutant of RBPj\{kappa\}. J Neurosci 2011; 31:8046-58.

112. Kistner A, Gossen M, Zimmermann F, Jerecic J, Ullmer C, Lubbert H, Bujard H. Doxycycline-mediated quantitative and tissue-specific control of gene expression in transgenic mice. Proc Natl Acad Sci U S A 1996; 93:10933-8.

113. Daudet N, Ariza-McNaughton L, Lewis J. Notch signalling is needed to maintain, but not to initiate, the formation of prosensory patches in the chick inner ear. Development 2007; 134:2369-78.

114. Pirvola U, Ylikoski J, Trokovic R, Hebert JM, McConnell SK, Partanen J. FGFR1 is required for the development of the auditory sensory epithelium. Neuron 2002; 35:671-80.

115. Hatch EP, Noyes CA, Wang X, Wright TJ, Mansour SL. Fgf3 is required for dorsal patterning and morphogenesis of the inner ear epithelium. Development 2007; 134:3615-25.

116. Jayasena CS, Ohyama T, Segil N, Groves AK. Notch signaling augments the canonical Wnt pathway to specify the size of the otic placode. Development 2008; 135:2251-61.

117. Ohyama T, Mohamed OA, Taketo MM, Dufort D, Groves AK. Wnt signals mediate a fate decision between otic placode and epidermis. Development 2006; 133:865-75.

118. Cohen DE, Melton D. Turning straw into gold: directing cell fate for regenerative medicine. Nat Rev Genet 2011; 12:243-52.

119. Takahashi K, Yamanaka S. Induction of pluripotent stem cells from mouse embryonic and adult fibroblast cultures by defined factors. Cell 2006; 126:663-76. 
120. Vierbuchen T, Ostermeier A, Pang ZP, Kokubu Y, Sudhof TC, Wernig M. Direct conversion of fibroblasts to functional neurons by defined factors. Nature 2010; 463:1035-41.

121. Ieda M, Fu JD, Delgado-Olguin P, Vedantham V, Hayashi Y, Bruneau BG, Srivastava D. Direct reprogramming of fibroblasts into functional cardiomyocytes by defined factors. Cell 2010; 142:375-86.

122. Zhou Q, Brown J, Kanarek A, Rajagopal J, Melton DA. In vivo reprogramming of adult pancreatic exocrine cells to beta-cells. Nature 2008; 455:627-32.

123. Liberman MC, Gao J, He DZ, Wu X, Jia S, Zuo J. Prestin is required for electromotility of the outer hair cell and for the cochlear amplifier. Nature 2002; 419:300-4.

124. Zheng J, Shen W, He DZ, Long KB, Madison LD, Dallos P. Prestin is the motor protein of cochlear outer hair cells. Nature 2000; 405:149-55.

125. Ayrault O, Zhao H, Zindy F, Qu C, Sherr CJ, Roussel MF. Atoh1 inhibits neuronal differentiation and collaborates with Gli1 to generate medulloblastoma-initiating cells. Cancer Res 2010; 70:5618-27.

126. Gomez-Casati ME, Murtie J, Taylor B, Corfas G. Cell-specific inducible gene recombination in postnatal inner ear supporting cells and glia. J Assoc Res Otolaryngol 2009; 11:19-26.

127. Avraham KB, Hasson T, Steel KP, Kingsley DM, Russell LB, Mooseker MS, Copeland NG, Jenkins NA. The mouse Snell's waltzer deafness gene encodes an unconventional myosin required for structural integrity of inner ear hair cells. Nat Genet 1995; 11:369-75.

128. Hasson T, Gillespie PG, Garcia JA, MacDonald RB, Zhao Y, Yee AG, Mooseker MS, Corey DP. Unconventional myosins in inner-ear sensory epithelia. J Cell Biol 1997; 137:1287-307.

129. Hackney CM, Mahendrasingam S, Jones EM, Fettiplace R. The distribution of calcium buffering proteins in the turtle cochlea. J Neurosci 2003; 23:4577-89. 
130. Hertzano R, Dror AA, Montcouquiol M, Ahmed ZM, Ellsworth B, Camper S, Friedman TB, Kelley MW, Avraham KB. Lhx3, a LIM domain transcription factor, is regulated by Pou4f3 in the auditory but not in the vestibular system. Eur J Neurosci 2007; 25:999-1005.

131. Shroyer NF, Wallis D, Venken KJ, Bellen HJ, Zoghbi HY. Gfi1 functions downstream of Math1 to control intestinal secretory cell subtype allocation and differentiation. Genes Dev 2005; 19:2412-7.

132. Nakamura T, Colbert MC, Robbins J. Neural crest cells retain multipotential characteristics in the developing valves and label the cardiac conduction system. Circ Res 2006; 98:1547-54.

133. Zheng L, Sekerkova G, Vranich K, Tilney LG, Mugnaini E, Bartles JR. The deaf jerker mouse has a mutation in the gene encoding the espin actin-bundling proteins of hair cell stereocilia and lacks espins. Cell 2000; 102:377-85.

134. Meyer AC, Frank T, Khimich D, Hoch G, Riedel D, Chapochnikov NM, Yarin YM, Harke B, Hell SW, Egner A, Moser T. Tuning of synapse number, structure and function in the cochlea. Nat Neurosci 2009; 12:444-53.

135. Szabo E, Rampalli S, Risueno RM, Schnerch A, Mitchell R, Fiebig-Comyn A, Levadoux-Martin M, Bhatia M. Direct conversion of human fibroblasts to multilineage blood progenitors. Nature 2010; 468:521-6.

136. Xie H, Ye M, Feng R, Graf T. Stepwise reprogramming of B cells into macrophages. Cell 2004; 117:663-76.

137. Chen P, Johnson JE, Zoghbi HY, Segil N. The role of Math1 in inner ear development: Uncoupling the establishment of the sensory primordium from hair cell fate determination. Development 2002; 129:2495-505.

138. Helms AW, Abney AL, Ben-Arie N, Zoghbi HY, Johnson JE. Autoregulation and multiple enhancers control Math1 expression in the developing nervous system. Development 2000; 127:1185-96.

139. Yang H, Gan J, Xie X, Deng M, Feng L, Chen X, Gao Z, Gan L. Gfi1-Cre knock-in mouse line: A tool for inner ear hair cell-specific gene deletion. Genesis 2010; 48:400-6. 
140. Klisch TJ, Xi Y, Flora A, Wang L, Li W, Zoghbi HY. In vivo Atoh1 targetome reveals how a proneural transcription factor regulates cerebellar development. Proc Natl Acad Sci U S A 2011; 108:3288-93.

141. Rose MF, Ren J, Ahmad KA, Chao HT, Klisch TJ, Flora A, Greer JJ, Zoghbi HY. Math1 is essential for the development of hindbrain neurons critical for perinatal breathing. Neuron 2009; 64:341-54.

142. Finlay BL, Darlington RB. Linked regularities in the development and evolution of mammalian brains. Science 1995; 268:1578-84.

143. Clancy B, Darlington RB, Finlay BL. Translating developmental time across mammalian species. Neuroscience 2001; 105:7-17.

144. Eminli S, Foudi A, Stadtfeld M, Maherali N, Ahfeldt T, Mostoslavsky G, Hock H, Hochedlinger K. Differentiation stage determines potential of hematopoietic cells for reprogramming into induced pluripotent stem cells. Nat Genet 2009; 41:96876.

145. Yang YP, Thorel F, Boyer DF, Herrera PL, Wright CV. Context-specific $\{$ alpha\}-to-\{beta\}-cell reprogramming by forced Pdx1 expression. Genes Dev $2011 ; 25: 1680-5$.

146. Li G, Reinberg D. Chromatin higher-order structures and gene regulation. Curr Opin Genet Dev 2011;21:175-86.

147. Creyghton MP, Cheng AW, Welstead GG, Kooistra T, Carey BW, Steine EJ, Hanna J, Lodato MA, Frampton GM, Sharp PA, Boyer LA, Young RA, Jaenisch R. Histone H3K27ac separates active from poised enhancers and predicts developmental state. Proc Natl Acad Sci U S A 2010; 107:21931-6.

148. Carey BW, Markoulaki S, Beard C, Hanna J, Jaenisch R. Single-gene transgenic mouse strains for reprogramming adult somatic cells. Nat Methods 2010; 7:56-9. 


\section{VITA}

Zhiyong Liu was born in Linying City, Henan Province at P.R.China in 1980. Starting at September 1, 1998, he studied as an undergraduate medical student in West China Medical Center of Sichuan University, with the major in Forensic Medicine. After achieving the degree of Bachelor of Medicine in June 2003, he continued his master degree focusing on Forensic Genetics in West China Medical Center of Sichuan University until June 2006.

In August 2006, he came to America and joined the Integrated Program in Biomedical Sciences at the University of Tennessee Health Science Center for a Ph.D degree. In July 2007, he joined Dr. Jian Zuo's laboratory in Department of Developmental Neurobiology in St.Jude Children's Research Hospital and conducted research in studying functions of the important inner ear cochlear developmental genes for auditory hair cell regeneration. He received his Doctor of Philosophy degree from the University of Tennessee Health Science Center in December of 2011. 